\title{
The influence of the gut
}

\section{microbiota on influenza}

\author{
vaccine-induced
}

immunity

Anna Mooney

A thesis submitted to Victoria University of Wellington in fulfilment of the requirements for the degree of Master of Biomedical Science 



\section{Abstract}

Currently, annual vaccination is widely considered the most effective method for preventing and controlling influenza virus infection. However, many individuals mount suboptimal immune responses to vaccination and the factors leading to poor immune responses are yet to be elucidated. Interestingly, it has been proposed that microorganisms that inhabit the intestinal tract, the gut microbiota, can profoundly influence many facets of the host immune system, including the strength of the immune response to influenza vaccination.

In line with these observations, we observed that short-term administration of antibiotics drastically reduced influenza vaccine-specific antibody production. In particular, antibiotic treatment diminished the frequency and activation status of multiple myeloid cell subsets in the draining lymph nodes at steady-state and following vaccination, with associated impairments in B and $\mathrm{T}_{\mathrm{FH}}$ cell responses.

Composition and function of gut microbiota communities can be rapidly altered through dietary changes. Therefore, the impact of potential prebiotic and probiotic nutritional interventions on the immune response to influenza vaccination and subsequent infection was assessed. No improvement in antibody responses to influenza vaccination was observed following the nutritional interventions studies. However, oral administration of a propolis formulation led to some improvement in viral control following infection.

Collectively, this investigation indicates that alterations in microbial-associated signals leads to severe impairments in cellular responses crucial to humoral immunity and subsequent vaccine-induced antibody production. Furthermore, by altering the gut microbiota through dietary interventions, there is potential to improve immune responses to vaccination. 


\section{Acknowledgements}

Thank you to my supervisors, Dr. Olivier Gasser and Dr. Elizabeth Forbes-Blom, for your guidance over the past two years. Liz, thank you for taking me on and for all you taught me during my first year. Oli, thank you for stepping in when I needed you, for giving me great opportunities to grow and giving me independence in my project, it has all helped me grow into a confident and independent researcher.

Thanks so much to everyone in the Translational Immunology team for the knowledge, support and guidance. In particular Angela, I am so grateful for all your support, especially in the past year. Thank you for helping me in the long hours in the BRU, always giving me words of encouragement when I needed them and just being an amazing friend, I could not have done this without you.

To the science staff at Malaghan who have helped and supported me throughout, there are too many to name but I have loved working with such a kind, intelligent and passionate group of people. Thank you in particular to Dr. Kara Filbey and Dr. Hazel Poyntz for all your feedback during the writing process.

All of the technical and support staff at the Malaghan Institute, thank you! Thanks to the HGCC for all the help, especially for support when everything possible goes wrong on the cytometer. To the BRU, thanks for all that you do and for creating a great environment to work in. To fundraising, in particular Yvonne and Jenny, thank you for getting me the support I needed so I could focus on my research, I would not have made it without you! To the finance, IT and building management teams, your work keeps MIMR running, thank you! 
Thank you to the Frimley Foundation, the Infinity Foundation and other funding bodies for their generous support of my research and travel throughout my study.

To the young researchers of the Malaghan Institute, past and present, you have made my time here incredible. In particular Emma, Olivia, Ruby, Jodie, Kirsty and Josh, I'm so lucky to have been able to study alongside you all, you truly have become the most amazing group of friends. Thanks for keeping me alive with many coffees, beers and chats. You are a bunch of beautiful, lovely and smart people and I cannot wait to see how far you all go.

To Elliott, Kayla and Kate, and all my friends far and wide, thank you for providing the love and fun that keeps me going.

To my family, Oliver, Isabel, Hugh and Joe, I'm so lucky to have the coolest and most inspiring siblings. To Dad, thank you for all you have done for me, your love and support has carried me to where I am today. I love you guys so much. Thanks to all of my aunties, uncles and cousins. In particular, Anna and Neal, thank you all your support while I have been in Wellington and being my second home, there was always a bed, a listening ear and some home-baked bread waiting for me when I needed it!

Finally, to my Mum, I hope I am making you proud. I miss you every day. 


\section{Disclosure Statement}

Dr. Hazel Poyntz performed and supplied the data for the long-term broad-spectrum antibiotic experiment.

With this disclosure, I declare that the content of this thesis is my own work. 


\section{Table of contents}

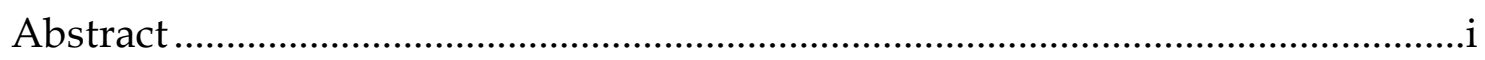

Acknowledgements........................................................................................... ii

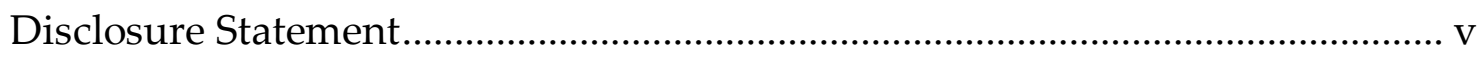

Table of contents ............................................................................................ vii

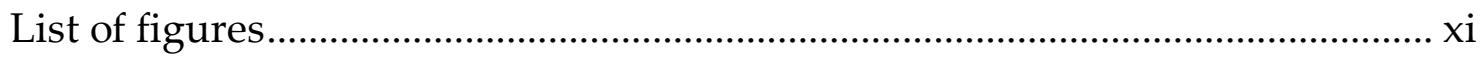

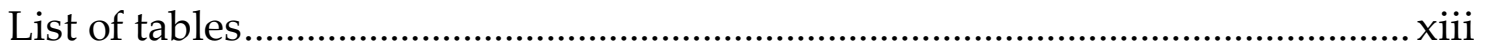

List of abbreviations ....................................................................................... xiv

1 General introduction ................................................................................

$1.1 \quad$ The humoral immune response...............................................................

1.1.1 Overview of the innate and adaptive immune systems ...........................2

1.1.2 Immunoglobulins .................................................................................

1.1.3 Generation of antibody-secreting cells .......................................................

1.1.4 Vaccination and adjuvants ......................................................................14

1.2 Influenza infection and vaccination.......................................................16

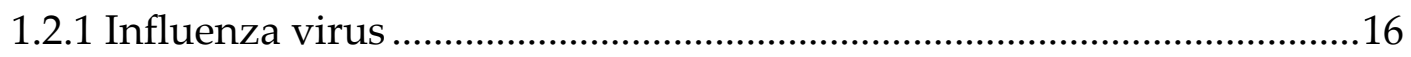

1.2.2 Influenza vaccination ............................................................................17

1.2.3 Immune response to influenza vaccines are suboptimal........................19

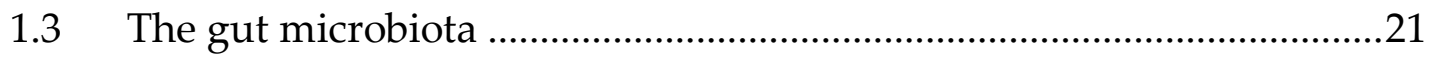

1.3.1 Microbiota community analysis ..............................................................22

1.3.2 The interface between the intestinal immune system and the microbiota

1.3.3 Gut microbiota interactions and the systemic immune system............24

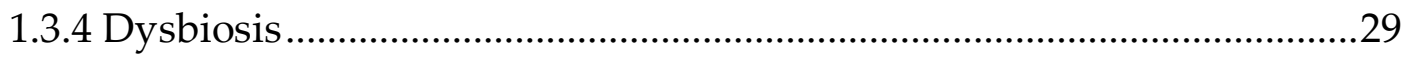

1.3.5 Beneficial modulation of the gut microbiota ..............................................30

$1.4 \quad$ Aims and hypotheses .............................................................................

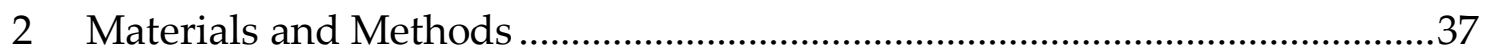

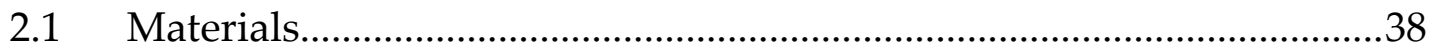




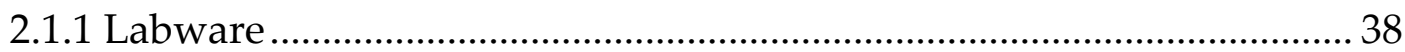

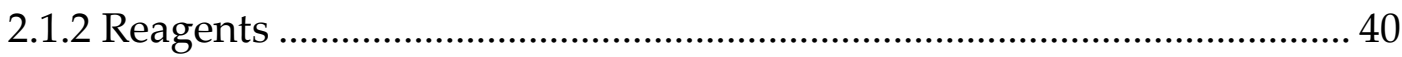

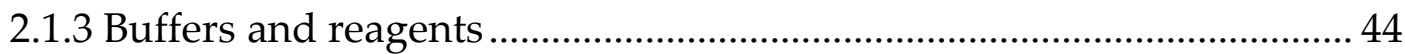

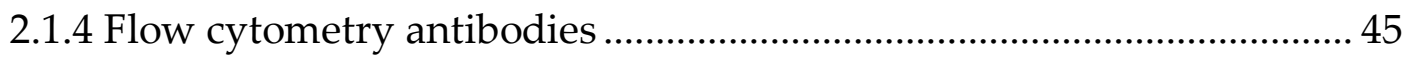

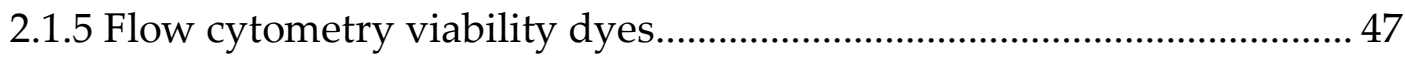

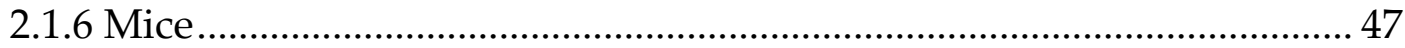

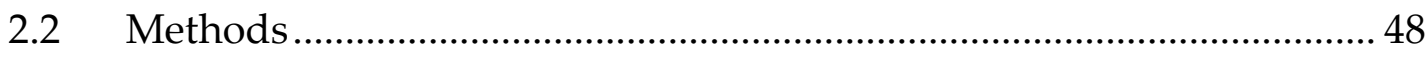

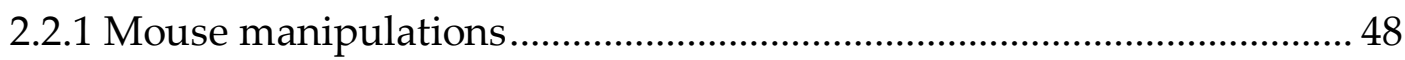

2.2.2 Tissue collection and processing ….................................................. 50

2.2.3 Production of inactivated viral vaccines .......................................... 53

2.2.4 Enzyme-Linked Immunosorbent Assay (ELISA) ............................... 54

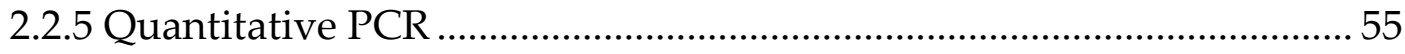

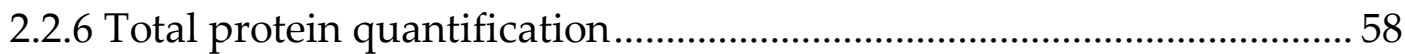

2.2.7 TIV vaccine AF488 protein labelling ................................................. 58

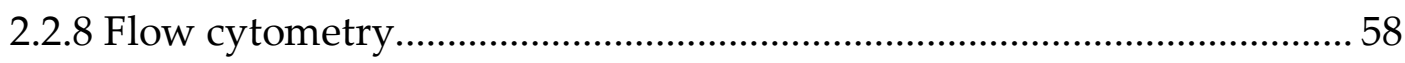

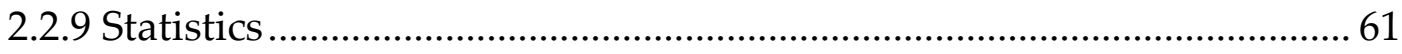

3 Evaluating the gut microbiota influence on antibody responses to influenza

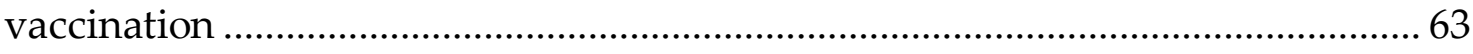

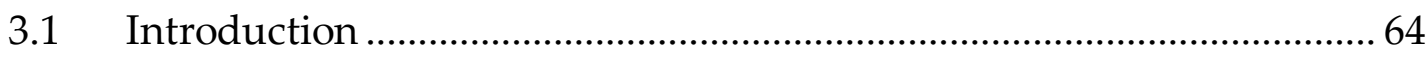

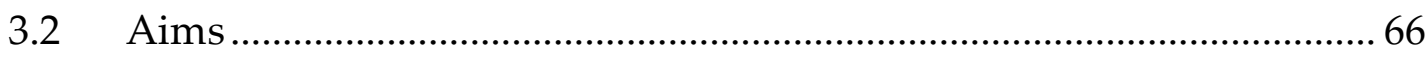

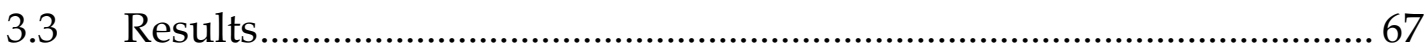

3.3.1 Intact microbiota communities are required for antibody response to

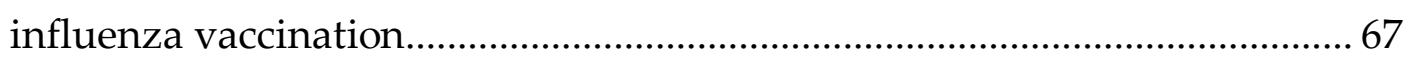

3.3.2 Subcutaneous TIV vaccination induces potent B cell responses in

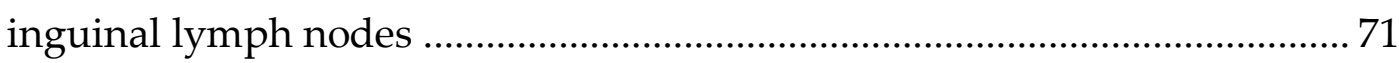

3.3.3 Antibiotic treatment impairs B cell differentiation ............................. 75

3.3.4 Antibiotic treatment reduces follicular helper cell population ............ 78 
3.3.5 AF488 labelled TIV vaccine fluorescently labels cells non-specifically in vivo

3.3.6 Antibiotic treatment impacts number and activation status of myeloid cells at steady-state

3.3.7 Antibiotic treatment impacts number and activation status of myeloid cells following vaccination

3.4 Discussion .92

3.5 Conclusion .98

4 The influence of functional foods on immune responses to influenza vaccination

4.1 Introduction .100

4.2 Aims. 102

4.3 Results 104

4.3.1 Formalin-inactivated influenza A vaccine does not induce strong antibody responses when administered SC or IN .104

4.3.2 UV-inactivated influenza A virus induces robust serum and mucosal antibody responses .108

4.3.3 Administration of inactivated influenza vaccines protect against infection.

4.3.4 Oral administration of kiwifruit does not improve antibody responses to influenza vaccination or protection from infection.

4.3.5 Oral administration of probiotic B. animalis does not enhance antibody responses to influenza vaccination or protect from infection. .118

4.3.6 Oral administration of propolis formulation improves protection against infection without altering antibody responses.

4.3.7 Dietary fibre intake does not alter antibody responses to vaccination or prevent infection-induced weight loss 


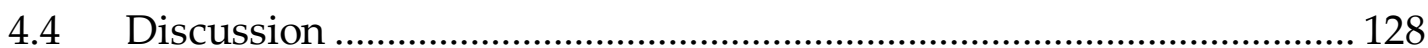

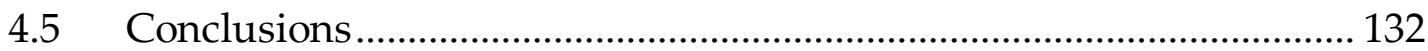

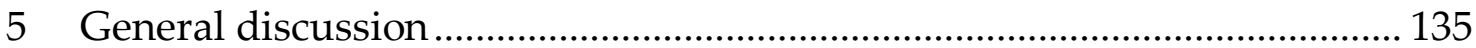

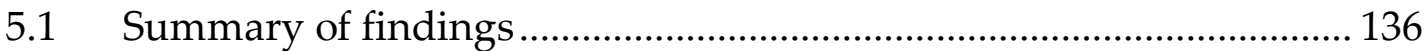

5.2 The microbiota influences multiple cell populations in the influenza

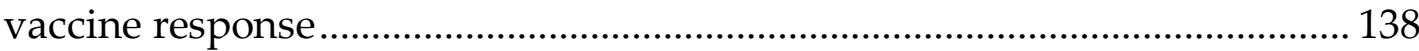

5.3 Enhancing influenza vaccine efficacy with functional foods ............. 142

$5.4 \quad$ The future of microbiota-targeted therapies ....................................... 143

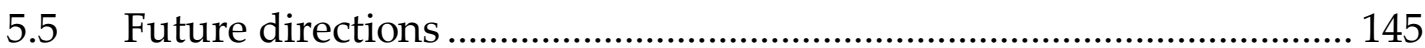

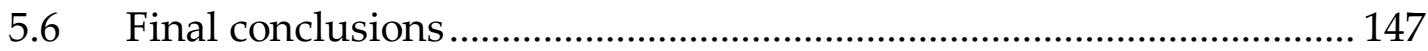

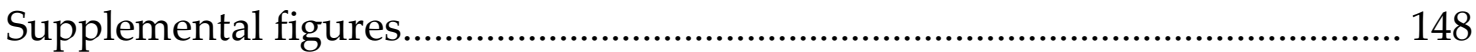

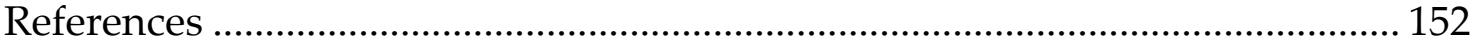




\section{List of figures}

Figure 1.1: Structure of an immunoglobulin molecule. ......................................6

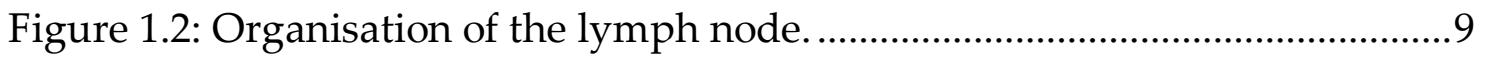

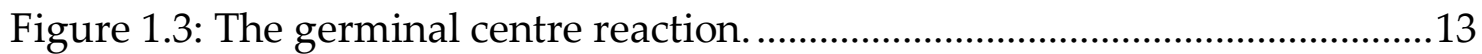

Figure 2.1: Pre-gating strategy for flow cytometry analysis. ..............................62

Figure 3.1: Administration of antibiotics is detrimental to vaccine-specific antibody production and significantly alters faecal microbial communities.....70 Figure 3.2: SC TIV vaccination at the tail base drains to inguinal LN and induces potent $\mathrm{B}$ cell responses at 14 days post vaccination. .73

Figure 3.3: Representative flow cytometry gating strategy for B cell and PC populations in the LNs.

Figure 3.4: Antibiotic treatment impairs multiple stages of B cell differentiation following TIV vaccination. .77

Figure 3.5: Representative flow cytometry gating strategy for TrH cells in the LNs.

Figure 3.6: Antibiotic treatment leads to a reduction in TFH cells in the dLN following vaccination.

Figure 3.7: Representative flow cytometry gating strategy for vaccine-labelled cells in the dLN.

Figure 3.8: Fluorescently labelled TIV vaccine is acquired by non-APC populations.

Figure 3.9: Representative flow cytometry gating strategy for myeloid cells in the LNs. .88

Figure 3.10: Antibiotic treatment decreases myeloid cell numbers and activation state.

Figure 3.11: Antibiotic treatment severely impacts myeloid cell frequencies and activation status following TIV vaccination. .91 
Figure 4.1: Formalin-inactivated influenza A vaccine does not induce sufficient antibody production when administered SC or IN. 107

Figure 4.2: UV-inactivated influenza A virus induces strong serum and mucosal antibody responses.

Figure 4.3: Parenteral and mucosal influenza vaccines induce protection against infection. 114

Figure 4.4: Administration of kiwifruit is detrimental to antibody responses to influenza vaccination and protection from infection. 117 Figure 4.5: Oral administration of $B$. animalis elicits has no effect on vaccine-induced antibody production or protection against infection. 120 Figure 4.6: Propolis formulation intake elicits some protection against influenza infection without influencing vaccine-induced antibody production. 124 Figure 4.7: Fibre intake does not influence antibody production to vaccination or protection against infection-induced weight loss. 127

Supplementary figure 1: qPCR analysis of faecal DNA in antibiotic-treated mice. 


\section{List of tables}

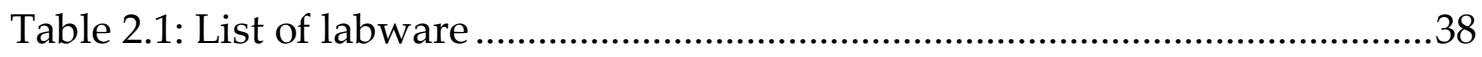

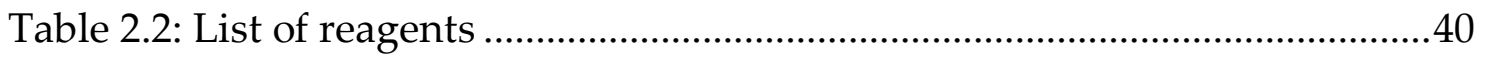

Table 2.3: List of flow cytometry antibodies ....................................................45

Table 2.4: List of flow cytometry viability dyes ..................................................47

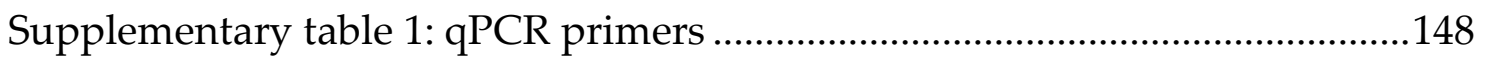




\section{Abbreviations}

ABX Antibiotics

ADCC Antibody-dependent cell-mediated cytotoxicity

Alum Aluminum salts

APC Antigen presenting cell

BAL Bronchoalveolar lavage

BCA Bicinchoninic acid

BCR B cell receptor

BM Bone marrow

BRU Biomedical Research Unit

C Constant

cDNA Complementary DNA

CFU Colony forming units

CLR C-type lectin receptor

CS Class-switched or class-switching

CSR Class-switch recombination

CTL Cytotoxic T lymphocyte

DAMP Danger-associated molecular pattern

DAPI 4',6-diamidino-2-phenylindole

DC Dendritic cell

dLN Draining lymph node

DMEM Dulbecco's Modified Eagle Medium

DZ Dark zone

ELISA Enzyme-linked immunosorbent assay

FBS Foetal bovine serum

FDC Follicular dendritic cell

GC Germinal centre

GF Germ-free 


\begin{tabular}{ll} 
GI & Gastrointestinal \\
GPCR & G-protein coupled receptor \\
H & Heavy \\
H.P.V. & Hours post-vaccination \\
HA & Haemagglutinin \\
HAU & Haemagglutination unit \\
HDAC & Histone deacetylase \\
HEV & High endothelial venule \\
HIV & Human immunodeficiency syndrome \\
IFN & Interferon \\
Ig & Immunoglobulin \\
IG & Intragastric or intragastrically \\
ILC & Innate lymphoid cell \\
IMDM & Iscove's Modified Dulbecco's Medium \\
IN & Intranasal or intranasally \\
L & Light \\
LAIV & Live attenuated influenza vaccine \\
LN & Lymph node \\
LNresDC & Lymph node-resident DC \\
LPS & Lipopolysaccharide \\
LZ & Light zone \\
MAC & Microbiota-accessible carbohydrates \\
MHC & Major histocompatibility complex \\
MigDC & Migratory DC \\
MIMR & Malaghan Institute of Medical Research \\
mRNA & Messenger ribonucleic acid \\
MW & Molecular-weight \\
M $\varphi$ & Macrophage or macrophages \\
NA & Neuraminidase \\
\hline
\end{tabular}


NK Natural killer

NLR Nucleotide-binding oligomerisation domain-like receptors

PAMP Pathogen-associated molecular pattern

PBMC Peripheral blood mononuclear cell

PBS Phosphate buffered saline

PC Plasma cell

PD-1 Programmed death receptor 1

PRR Pattern recognition receptor

RLR RIG-I-like receptors

RNA Ribonucleic acid

rRNA Ribosomal ribonucleic acid

RT Room temperature

SC Subcutaneous or subcutaneously

SCS Subcapsular sinus

SCFA Short-chain fatty acid

SCID Severe combined immunodeficiency

SFB Segmented filamentous bacteria

SHM Somatic hypermutation

sIgA Secretory IgA

SPF Specific-pathogen free

TCR T cell receptor

TrH $\quad$ T follicular helper

TFR T follicular regulatory

$\mathrm{T}_{\mathrm{H}} \quad$ Thelper

TH1 Thelper 1

TH2 Thelper 1

TH17 Thelper 17

TIV Trivalent influenza vaccine

TLR Toll-like receptor 
TREG Regulatory $\mathrm{T}$

V Variable

VNAM Vancomycin, neomycin, ampicillin and metronidazole 

1 General introduction 


\subsection{The humoral immune response}

\subsubsection{Overview of the innate and adaptive immune systems}

The immune system is a dynamic and diverse collection of cells and molecules, enabling protection against a wide variety of foreign insults. It consists of two arms, the innate and the adaptive immune systems, which sense the environment through receptors on, or inside, cells.

The innate immune response is rapid and a sufficient response can detect and eliminate pathogens in minutes to hours ${ }^{1}$. The innate immune system primarily consists of an array of myeloid cells and innate lymphoid cells (ILCs), which provide initial discrimination between pathogens and innocuous stimuli. Innate immune cells possess pattern-recognition receptors (PRRs), which encompass a limited number of germ-line encoded receptors including toll-like receptors (TLRs), nucleotide-binding oligomerisation domain-like receptors (NLRs) RIG-I-like receptors (RLRs) and C-type lectin receptors (CLRs) ${ }^{2}$. These are able to recognise pathogen- and damage-associated molecular patterns (PAMPs and DAMPs, respectively). PAMPs are molecular motifs that are conserved between a wide variety of pathogens ${ }^{3}$, while DAMPs are endogenous molecules released from host cells following stress and injury ${ }^{4}$ Binding of PRRs by PAMPs or DAMPs alerts innate immune cells to infectious threats and associated tissue damage, giving rise to effector responses. These rapid, antigen-independent responses include the engulfing and killing of pathogens, production of toxic molecules to directly kill or damage pathogens and secretion of molecules to recruit other innate immune cells to the site of infection ${ }^{1}$.

The adaptive immune system mainly consists of lymphocytes that can be broadly divided into two groups: $\mathrm{T}$ and $\mathrm{B}$ cells. Both possess unique antigen receptors that are generated through rearrangement of their germline receptor 
sequences, giving rise to antigen receptors with random specificity, enabling recognition of an immense diversity of pathogen-derived products ${ }^{5}$. Adaptive immune cells exert highly specific responses against invading pathogens. T cells orchestrate protective immune responses and directly kill infected cells, while B cells produce antibodies to neutralize and eliminate pathogens from the body. Unlike innate immune cells, after resolution of the infection, cells of the adaptive immune system can contribute to antigen-specific immunological memory. A small proportion of $\mathrm{T}$ and B cells can acquire a 'memory' phenotype where upon subsequent exposure to a pathogen, they mount a more rapid and powerful response than the primary response ${ }^{6,7}$.

Conventional $\mathrm{T}$ cells can be categorised by effector function and expression of either CD4 or CD8 molecules. Each T cell has a unique T cell receptor (TCR) that recognises and binds to antigen presented on major histocompatibility (MHC) molecules by antigen presenting cells (APCs). $\mathrm{CD}^{+} \mathrm{T}$ cells recognise antigen that is presented on MHC class I and differentiate into cytotoxic T lymphocytes (CTLs), specialising in direct killing of infected cells through secretion of toxic molecules such as granzyme and perforin ${ }^{8}$. In contrast, $\mathrm{CD} 4^{+} \mathrm{T}$ cells recognise antigen presented on MHC class II and differentiate into a number of distinct T helper $\left(\mathrm{T}_{\mathrm{H}}\right)$ cell subtypes, defined by their distinct cytokine profiles and effector functions. The major subsets are $\mathrm{T}_{\mathrm{H}} 1, \mathrm{~T}_{\mathrm{H}} 2, \mathrm{~T}_{\mathrm{H}} 17$, regulatory $\mathrm{T}\left(\mathrm{T}_{\mathrm{REG}}\right)$ and $\mathrm{T}$ follicular helper $\left(\mathrm{T}_{\mathrm{FH}}\right)$ cells. $\mathrm{T}_{\mathrm{H}} 1$ cells are key effector cells in intracellular infection and produce IFN- $\gamma$ and IL-2, activating macrophages and inducing effector function of CTLs ${ }^{9}$. TH2 cells produce IL-4, IL-5 and IL-13 and orchestrate protection against multicellular pathogens, such as helminths, by recruiting and activating mast cells, basophils, eosinophils and directing the production of appropriate antibodies ${ }^{10}$. IL-17 producing $\mathrm{T}_{\mathrm{H}} 17$ cells play a key role in control of bacterial and fungal infections by recruiting and activating neutrophils ${ }^{11}$. TREG cells produce IL-10 and TGF- $\beta$ and are important in resolving inflammation and 
preventing inappropriate immune responses ${ }^{12}$. TFH cells produce IL-6 and IL-21 and provide key signals to B cells for effective antibody responses ${ }^{13}$.

Innate immune cells play a critical role in the initiation and regulation of adaptive immune responses. T cells are unable to effectively respond to free antigen. Instead, antigen must be presented in the context of MHC with multiple secondary co-stimulatory signals from the APC to prime the $\mathrm{T}$ cell. The most effective APCs are dendritic cells (DCs), but macrophages $(\mathrm{M} \varphi)$ and B cells may also present antigen. DCs have an immature phenotype in the periphery. Upon recognition of pathogens via PRRs, DCs take up antigen and mature to an activated phenotype characterised by upregulation of costimulatory molecules, such as CD80 and $86^{14}$, as well as CCR7, which promotes cellular migration to secondary lymphoid tissues ${ }^{15}$. Here, DCs present antigen via MHC molecules to the TCR and crucial CD80/86 costimulation to CD28 on T cells ${ }^{14}$. DCs also provide additional signals, such as cytokines and chemokines, which direct the differentiation of $\mathrm{T}$ cells to a subset appropriate to the pathogenic environment ${ }^{16}$. This stringent process of $\mathrm{T}$ cell activation ensures that $\mathrm{T}$ cells are activated only if the antigen they present is pathogen-derived. The requirement for the MHCTCR complex, CD80/86-CD28 co-stimulation and cytokine production provides crucial checkpoints that prevent inflammatory immune responses being mounted against innocuous environmental or self-antigens as well as driving specific immune responses that are appropriate for control of the infectious challenge.

\subsubsection{Immunoglobulins}

Pathogens invade the body via disruptions to epithelial barriers, by crossing host mucosal surfaces that line the respiratory, urogenital and gastrointestinal tracts, or, less commonly, by direct entry into the blood. Once infection has occurred, pathogens can also inhabit extracellular spaces of the body. In these scenarios, 
infections can be controlled by antibodies secreted by B cells into extracellular spaces, termed the humoral immune response. Antibodies are antigen-specific proteins produced following immunisation, either through infection or vaccination, that bind cognate antigen, eliciting a variety of effector functions to aid in long-lasting protection against infection.

\subsubsection{Immunoglobulin structure}

Immunoglobulins (Igs) are exclusively produced by B cells and can be found in two forms, either as soluble antibodies which are present in blood and extracellular fluids and secreted across mucosal barriers, or as membrane-bound B cell receptors (BCRs). Igs consist of two heavy $(\mathrm{H})$ chains linked by disulfide bonds and each heavy chain is also linked via a disulfide bond to a light (L) chain. These can be further subdivided into variable $(\mathrm{V})$ regions and constant $(\mathrm{C})$ regions (also known as Fc regions) ${ }^{17}$ (Figure 1.1). The amino acid sequence of the $\mathrm{V}$ regions are highly variable between different Ig molecules and this confers the antigen specificity of the molecule. The genome of each individual possesses many different gene segments that encode the $\mathrm{V}$ region of Igs. During B cell development in the bone marrow (BM), gene segments are randomly spliced together generating an extensive potential pool of B cells, each with unique variable regions that encode an $\mathrm{Ig}$ with an individual antigen specificity, a process known as somatic recombination ${ }^{5}$. Following antigen exposure, the Ig will be further diversified through somatic hypermutation (SHM), a process of random mutation in the $\mathrm{V}$ region antigen binding pocket ${ }^{18}$. This generates further variation in Ig specificity and enables B cells to recognise a wide range of invading pathogens that the individual may encounter throughout its lifetime. The $\mathrm{C}$ regions show far less variability between $\mathrm{Ig}$ molecules and determine the antibody isotype and, accordingly, its effector function ${ }^{19}$. The isotype, or class, of the Ig may change over the course of the immune response through a process called class-switch recombination (CSR). The Ig heavy chain locus consists of 
distinct gene clusters, encoding the five $\operatorname{Ig}$ isotypes: $\operatorname{IgM}, \operatorname{IgD}, \operatorname{IgG}, \operatorname{IgE}$ and $\operatorname{Ig} A$. During CSR, segments of this locus are deleted, bringing the promoter in line with one of the other isotype clusters, leading to sole expression of the appropriately switched $\operatorname{Ig}^{20}$. Ig gene rearrangement, SHM and CSR allow B cells to alter both the specificity of the antibody and its effector function to effectively eliminate pathogens.

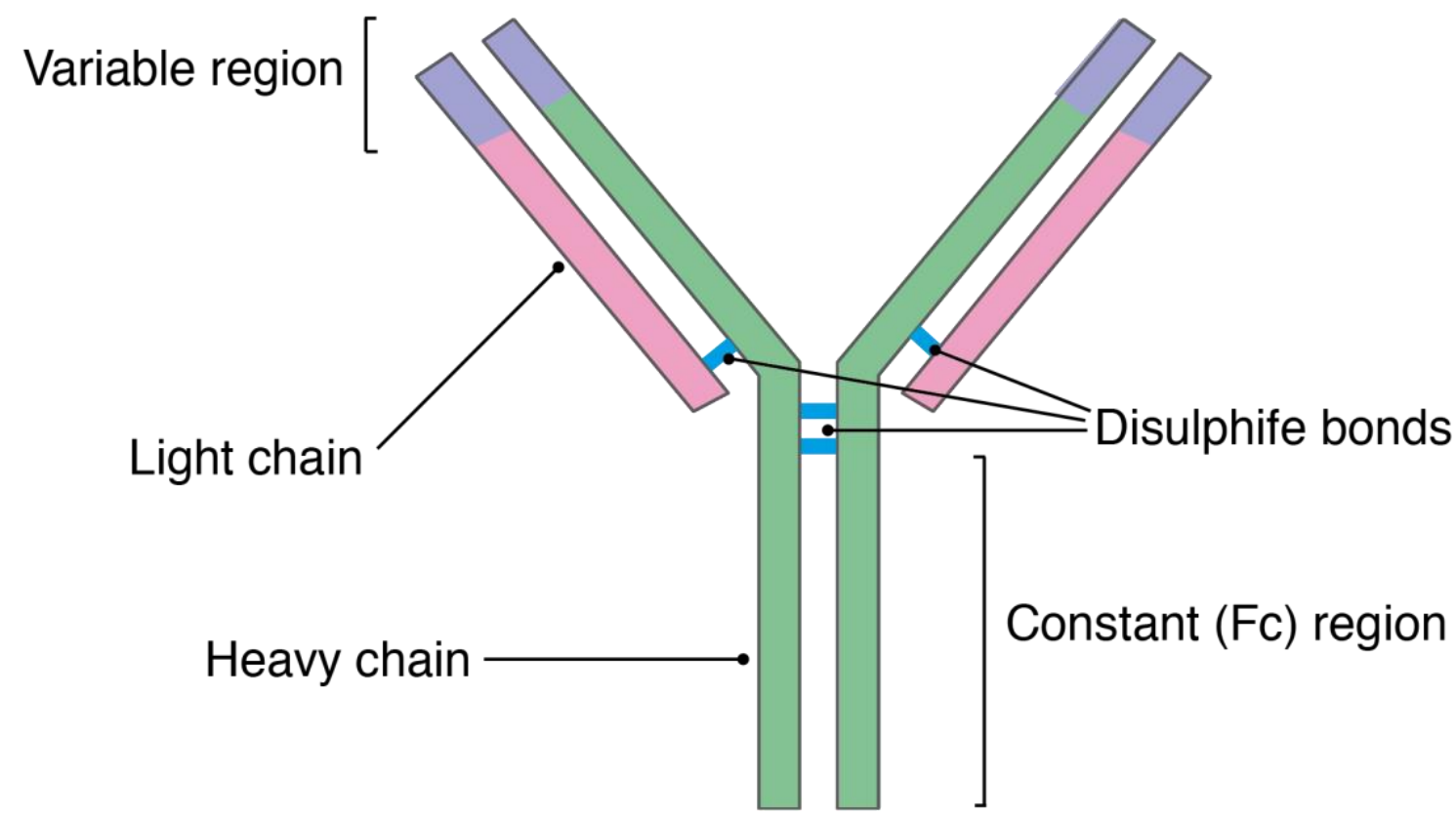

Figure 1.1: Structure of an immunoglobulin molecule. Immunoglobulins consist of two heavy (H) chains paired with two light (L) chains, which can be further subdivided into variable (V) regions and constant $(\mathrm{C})$ or Fc regions. Chains are linked together via disulfide bonds.

\subsubsection{Antibody effector functions}

Different antibody isotypes are made in distinct sites and have varying effector functions and the isotype that dominates a response will differ according to the immunising agent and where it enters the body. Mature naïve B cells co-express $\operatorname{IgD}$ and IgM as surface $\operatorname{Igs}^{21}$. Upon encounter of their cognate antigen, activated B cells lose expression of IgD. Some will retain IgM expression, while others undergo CSR to express IgG, IgE or IgA ${ }^{20}$. IgM is the first antibody produced in 
the humoral response and circulates in the blood as a pentamer. IgM is a strong activator of the complement cascade, which facilitates lysis and phagocytosis of pathogens and is important in controlling infections in the bloodstream ${ }^{22}$. IgG is the predominant antibody in the blood and can be divided into four subtypes, IgG1, IgG2, IgG3 and IgG4, which are differentially produced in response to varying antigens ${ }^{23}$. A major role of $\operatorname{IgG}$ is neutralisation: it can bind pathogen surfaces, preventing invasion of host cells, but is also able to activate complement ${ }^{24}$ IgG antibodies can also bind virally infected cells and mediate antibody-dependent cell-mediated cytotoxicity (ADCC), where the Fc portion binds to the Fc receptor, Fc $\gamma$ RIII, on natural killer (NK) cells, triggering release of cytotoxic granules to kill infected cells ${ }^{1}$. IgA constitutes $70 \%$ of Ig produced by mammals and is mostly secreted at mucosal surfaces ${ }^{25}$, where it's primary function is to neutralise pathogens, preventing access to the mucosa ${ }^{26,27}$. IgE is present at low peripheral concentrations and can be found primarily bound to granulocytes (mast cells and basophils) via FceRI in the tissues. When antigen binds and cross-links IgE on the surface of granulocytes, they degranulate and release an array of pre-formed toxic mediators ${ }^{28}$, a process that plays a key role in eliminating parasitic worm infections, as well as in the pathogenesis of allergies and asthma ${ }^{28}$.

\subsubsection{Generation of antibody-secreting cells}

\subsubsection{Antigen transport to the draining lymph node}

The generation of high affinity, class-switched antibodies during infection or vaccination requires a concerted effort between multiple cell types across the innate and adaptive immune systems. Peripheral lymphoid organs, such as the lymph nodes (LNs), serve as a convergence point for lymphatic drainage from the tissues and are the location of many crucial immune processes, including the generation of antibody-secreting cells. LNs are spread widely throughout the 
body and are highly compartmentalized in order to facilitate effective interactions between lymphocytes and antigen.

Lymphatic drainage transports foreign antigen deposited in the tissue by infection or vaccination to the LN. Antigen must reach the follicle to be recognised by $\mathrm{B}$ cells, and the paracortex for recognition by $\mathrm{T}$ cells. Antigen reaches the subcapsular sinus (SCS) of the tissue draining LN (dLN) via afferent lymphatic vessels, transported here either by migrating APCs that have taken up antigen in the tissue, or as soluble antigen that has freely drained from the tissue $^{29}$ (Figure 1.2). From here, low molecular-weight (MW) soluble antigens may diffuse into the follicle through pores in the $\mathrm{SCS}^{30}$ or via conduits ${ }^{31}$. Alternately, $\mathrm{M} \varphi$ are able to transport antigens from the SCS into the underlying follicle $^{32}$. Antigen may also drain to the medulla where LN-resident DCs (LNresDC) are able to transport antigen to the follicle ${ }^{33}$. On the other hand, antigen destined for the $\mathrm{T}$ cell paracortex is transported here by migratory $\mathrm{DC}$ (migDC) populations ${ }^{34}$ or low MW antigens may also travel via the conduit network in the T cell area ${ }^{35}$, where LNresDCs can sample antigen ${ }^{36}$. 


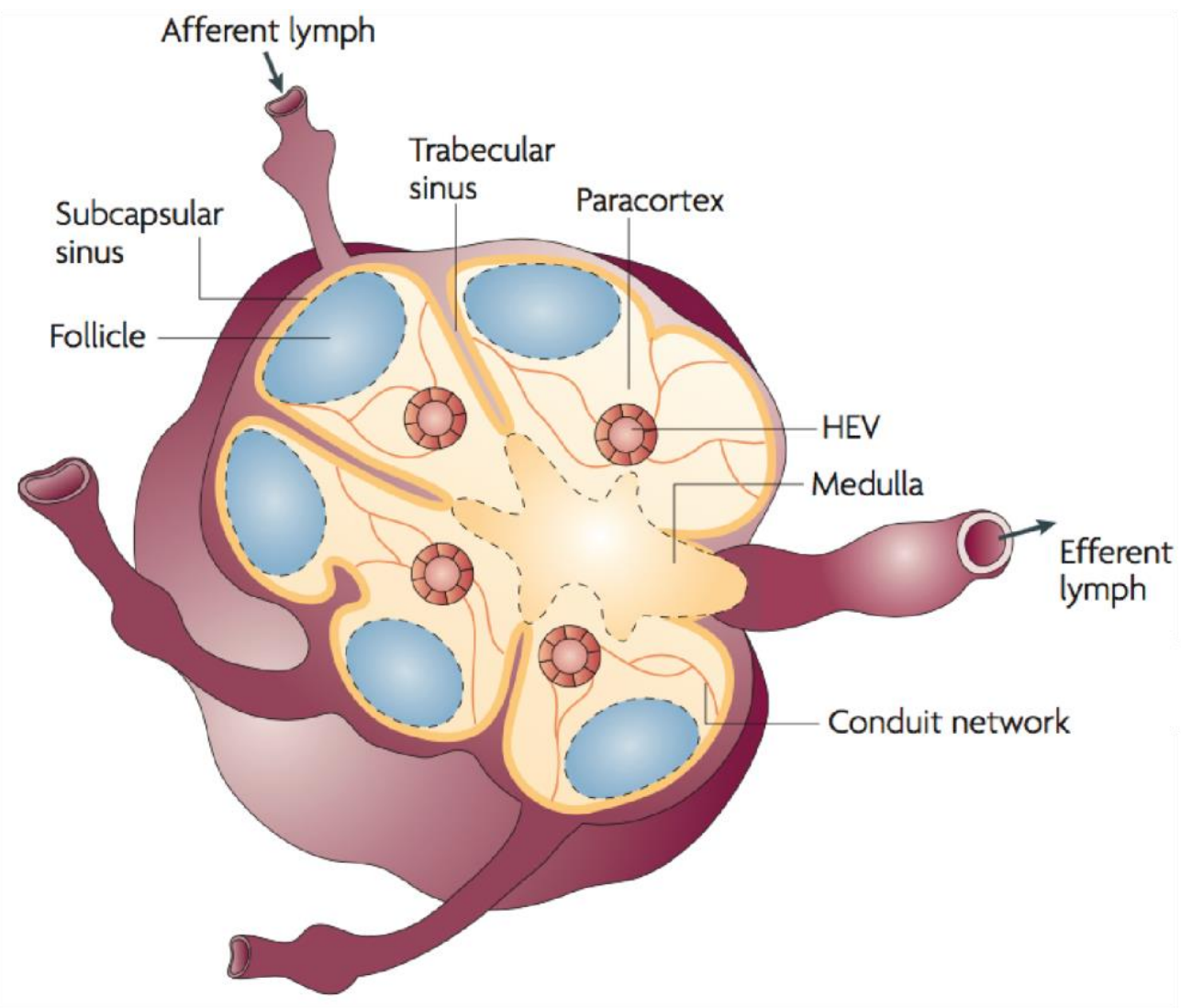

Figure 1.2: Organisation of the lymph node. The lymph node can be divided into three major regions: the medulla, the paracortex ( $\mathrm{T}$ cell zone) and the follicles (B cell zones). Naïve T and B cells circulate the body, entering the LN via HEVs and B cells migrate to the follicles while T cells assemble in the paracortex. Afferent lymph drains from the tissues, carrying immune cells and soluble antigen, into the SCS. From here, antigen can enter the follicle through pores in the SCS. Antigen also enters the conduit network where it can enter the follicles or paracortex. Figure from Batista et al. $2009^{37}$

\subsubsection{Lymphocyte activation in the lymph node}

Under homeostatic conditions, mature naïve $\mathrm{T}$ and $\mathrm{B}$ cells constitutively circulate between secondary lymphoid organs ${ }^{38}$. Upon reaching the LN, B and $\mathrm{T}_{\mathrm{H}}$ cells exit the blood via high endothelial venules ${ }^{39}$, with B cells migrating to follicles and $\mathrm{T}_{\mathrm{H}}$ cells entering the surrounding paracortex in pursuit of antigen ${ }^{40}$. Following antigen exposure, DC populations present antigen and provide costimulation to effectively activate naïve $\mathrm{T}$ cells, which proliferate and migrate to the interface between the follicle and paracortex (T-B border) ${ }^{41}$. In parallel, B cells collect soluble antigen or antigen from APCs on surface Ig, which they 
internalize and migrate to the T-B border ${ }^{41}$. This brings the two rare populations of naïve cognate $\mathrm{B}$ and $\mathrm{T}_{\mathrm{H}}$ cells into proximity, allowing stable cell-cell interactions to be established ${ }^{42}$. Here, B cells present processed antigen via MHC II to $\mathrm{T}_{\mathrm{H}}$ cells, which reciprocally provide crucial signals for B cell survival and differentiation, such as CD40L ${ }^{43}$. B cells compete for limited $\mathrm{T}_{\mathrm{H}}$ cell help and only those B cells possessing Igs with highest antigen affinity will survive and progress.

Selected B cells have three potential fates. A subset of activated B cells will become early memory $\mathrm{B}$ cells ${ }^{44}$. Others will migrate to the outer follicle and undergo an extrafollicular response, where they differentiate into short-lived plasma cells (PCs). These secrete low-affinity antibody early following immunisation for rapid protection against infection, but only survive for around three days ${ }^{45}$. The third option is the re-entry of B cells to the follicle where they proliferate to form the germinal centre (GC), a dynamic structure that facilitates the generation of high-affinity, class-switched long-lived PCs and memory B cells ${ }^{7}$.

Importantly the $\mathrm{CD}^{+} \mathrm{T}_{\mathrm{H}}$ cell subset, $\mathrm{T}_{\mathrm{FH}}$ cells, is required for B cell development in the $\mathrm{GC}^{13}$. TFH cells are driven by the master regulator transcription factor, Bcl6, and are commonly characterised by the expression of programmed death receptor 1 (PD-1), the chemokine receptor CXCR5 and the secretion of IL-2 $1^{13}$. $T_{F H}$ cell development occurs at multiple stages in the LN. Initially, naïve $T_{H}$ must be primed with antigen and costimulatory signals from DCs, prompting differentiation into pre- $\mathrm{T}_{\mathrm{FH}}$ cells ${ }^{46,47}$. These precursors then receive further signals from $B$ cells for the full commitment to the $T_{F H}$ lineage ${ }^{48,49}$. TFH cells provide important signals to aid B cell activation, proliferation differentiation and survival ${ }^{13}$. This includes TFH cell CD40L binding to CD40 expressed by B cells, which is a critical signal to multiple stages of B cell activation and differentiation, 
as well as cytokine secretion (e.g. IL-21), which potently promotes GC B cell CSR and differentiation into PCs ${ }^{50,51}$.

\subsubsection{The germinal centre reaction}

Following immunisation the affinity of antibodies to antigen progressively increases, a selective process occurring in the GC that can be compared to Darwinian selection. Following activation, B cells rapidly divide in the follicle to form the GC, where they are identified by their expression of GL7 antigen ${ }^{52}$. By around day seven following immunisation, a mature GC can be observed, characterised by two functionally distinct areas: the dark zone (DZ) and light zone (LZ), which B cells migrate between during maturation (Figure 1.3). Ig diversification in B cells occurs in the DZ. Here, point mutations are introduced to $\mathrm{V}$ region genes by SHM, generating mutant $\mathrm{B}$ cell clones with altered antigen specificity $^{53}$ (Figure 1.3A). Indiscriminate mutations alter the affinity of the B cell Ig for the cognate antigen, so that B cells have varying affinities for the immunising antigen. These cells then move to the LZ where surface Ig affinity is tested in two stages (Figure 1.3B). Follicular dendritic cells (FDCs) of the LZ retain immunising antigen on their surface for an extended period of time ${ }^{54}$, allowing only the B cells expressing functional antigen-specific surface Ig to bind and endocytose antigen, which induces survival signals within the cell, whilst others apoptose (Figure 1.3C). Secondly, the density at which B cells present antigen on MHC II is proportional to antigen affinity of the surface Ig. Those presenting higher density antigen successfully compete for the limited $\mathrm{T}_{\mathrm{FH}}$ cell help, while lower affinity or non-functional mutants are neglected and apoptose $^{55,56}$ (Figure 1.3C). B cells can undergo multiple successive rounds of Ig diversification and selection, developing a population of high-affinity B cells ${ }^{7}$ (Figure 1.3F). Additionally, CSR in B cells occurs in the LZ and is largely dependent on $\mathrm{T}_{\mathrm{FH}}$ cell engagement of $\mathrm{B}$ cell $\mathrm{CD} 40^{57}$, along with cytokine 
signaling, that dictates the antibody isotype - such as IL-4, which promotes switching to IgE and IgG158 (Figure 1.3E).

High affinity B cell clones will receive selection signals from $\mathrm{T}_{\mathrm{FH}}$ cells, exit the GC and differentiate into memory B cells or long-lived PCs (Figure 1.3G and H), both of which may persist over a lifetime ${ }^{59,60}$. Long-lived PCs migrate to the BM and constitutively secrete antibody, providing a first line of defence against subsequent infection ${ }^{61}$. Furthermore, memory B cells can be rapidly reactivated upon antigen encounter to proliferate and produce large quantities of class-switched, high-affinity antibody ${ }^{62}$. 


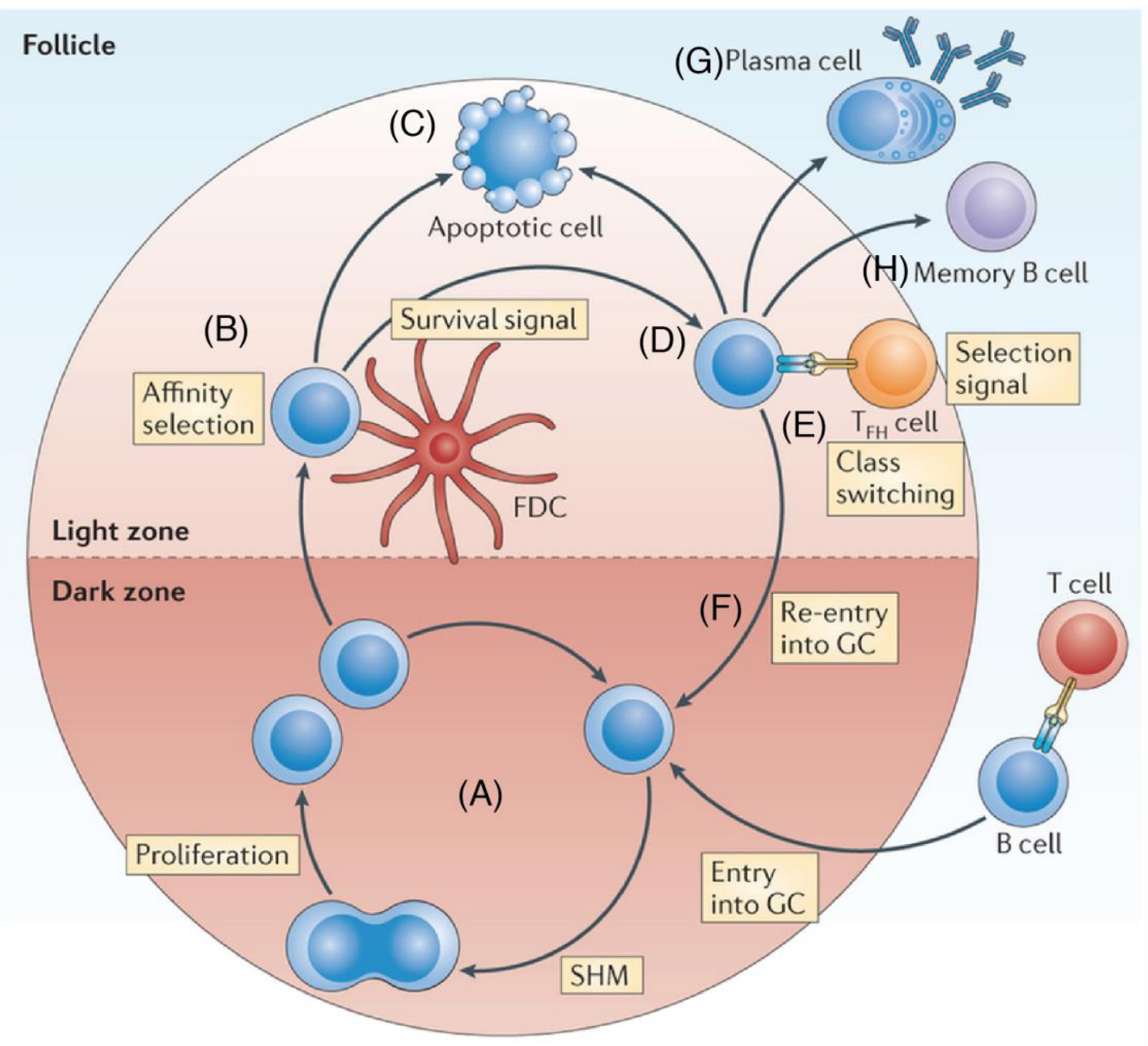

Figure 1.3: The germinal centre reaction. The GC can be divided into two functionally distinct regions, the dark and light zones. (A) In the dark zone, activated B cells generate mutant clones through SHM. (B) Mutants move to the light zone and recognise the immunising antigen retained on FDCs, only B cells expressing functional, antigen-specific Igs receive survival signals, (C) whilst others apoptose. (D) Selected B cells then compete for TFн cell help, only those with the greatest antigen affinity receive selection signals, whereas others apoptose. Cells then either (F) re-enter the dark zone for successive rounds of SHM, or differentiate into (G) PCs or (H) memory B cells. Figure adapted from Heesters et al.63. 


\subsubsection{Vaccination and adjuvants}

In addition to immunity conferred by natural infection, adaptive immunity to an infectious agent can be achieved by vaccination, whereby exposure to pathogen antigens in a non-virulent form induces immunological memory that protects from future pathogen exposure ${ }^{1}$. The majority of current vaccines elicit protection via the induction of antibodies. A number of vaccines induce extremely effective antibody responses and have been successful in eradicating several infectious diseases from industrialised countries, notably polio and smallpox ${ }^{64}$. Long-term protection is conferred by the persistence of high titres of pathogen-specific antibody or the maintenance of memory B cell populations ${ }^{65}$.

Vaccines can be either live attenuated, where the pathogen is weakened to reduce its virulence, or non-live vaccines, consisting of inactivated microorganisms or purified pathogen components, usually proteins. Live attenuated vaccines contain organisms that are able to replicate and maintain many of the pathogen-associated signals that are provided by a live infection, triggering the activation of multiple PRRs to elicit a strong immune response ${ }^{66}$. Non-live vaccines are a safer alternative, as no live organisms remain, however, most pathogen proteins are poorly immunogenic in the absence of danger signals provided by the live pathogen.

To address low immunogenicity, vaccines may contain an adjuvant, which provides additional 'danger signals' to increase immunogenicity of pathogen proteins ${ }^{67}$. Licensed adjuvants that boost antibody responses to vaccines include microbial particles, such as the LPS-derived molecule monophosphoryl lipid A (MPL), aluminum salts (alum) or oil-in-water emulsions, such as MF5968. The major function of vaccine adjuvants is to enhance the overall magnitude and affinity of vaccine-induced antibodies ${ }^{69,70}$, which often means that lower or fewer doses of the vaccine are required to achieve sufficient protective antibody titres 
termed seroconversion. Additionally, adjuvants may also be used to guide the type of adaptive immune response that ensues following vaccination ${ }^{67}$.

While adjuvants are in clinical use and prove extremely effective, the mechanisms of action are incompletely understood. It was thought until recently that the primary mechanism of most adjuvants was to act as a depot for vaccine antigen in the tissue, thereby ensuring slow release and prolonged immune stimulation $^{71}$. However, a number of studies have challenged this paradigm. For example, removal of the alum depot following vaccination has no effect on antibody production ${ }^{72}$ and it is now understood that adjuvants likely trigger innate immune responses more directly ${ }^{71}$. Many adjuvants induce a strong local inflammatory response. MF59 is able to recruit immune cells to the site of vaccination, facilitating antigen transport to the $\mathrm{dLN}^{73,74}$, as well as enhancing uptake of antigen by $\mathrm{APCs}^{75}$. Another key role of many adjuvants is to activate cells through PRRs, including the TLRs, NLRs, RLRs and CLRs. Once the receptor is bound, intracellular signalling leads to the activation of key transcription factors, such as NF-kB, IRF-3 and IRF-7, which ultimately results in the induction of genes encoding chemokines and cytokines, as well as inducing DC maturation and expression of costimulatory and MHC molecules ${ }^{76}$. MPL, for example, mediates adjuvant function via signaling through TLR $-4^{77}$. Studies suggest that some adjuvants, particularly alum, signal through NLRs to activate the inflammasome pathway ${ }^{78}$. The inflammasome is a protein complex that forms following PRR activation and is able to act on cytokine precursors to convert them to an active form, including the proinflammatory cytokines IL-1 $\beta$ and IL-18 ${ }^{78}$. Thus, adjuvants act via a multitude of immune pathways and evidence suggests that current effective adjuvants engage multiple mechanisms to enhance the humoral immune response to vaccination. 


\subsection{Influenza infection and vaccination}

\subsubsection{Influenza virus}

Influenza is an acute respiratory virus from the Orthomyxoviridae family. Influenza infection is a significant health burden worldwide, with seasonal epidemics estimated to cause 3 to 5 million infectious cases, leading to 250,000 to 500,000 deaths per year ${ }^{79}$. The virus infects the respiratory tract of humans and a number of other mammalian species and can cause both seasonal periods of illness and periodic pandemic outbreaks ${ }^{80}$. In the 2015 SHIVERS survey, influenza virus was found to infect $26 \%$ of the New Zealand population in a given influenza season, of whom $20 \%$ were symptomatic ${ }^{81}$. Influenza infection clinically manifests as a high fever, cough, headache, malaise and respiratory tract inflammation, among other symptoms, which may lead to severe complications, including pneumonia, secondary bacterial infection and hemorrhagic bronchitis ${ }^{82}$. Furthermore, certain groups of the community show greater susceptibility to infection and subsequent complications, including young children, pregnant women, the elderly and people with chronic comorbidities, such as diabetes ${ }^{83}$. Given the high incidence and risk of serious complications and mortality from influenza virus infection, effective prevention strategies are required.

The three major genera of influenza virus capable of causing human illness, influenza A, B and C, are distinguished by their distinct protein expression ${ }^{84}$. Seasonal epidemics are largely caused by types A and B, with influenza C causing only mild respiratory illness ${ }^{85}$. The viruses negative-sense ribonucleic acid (RNA) segments are packaged into ribonucleosome complexes and encased in a host-derived lipid envelope. A number of proteins are embedded in the envelope, most notably the glycoproteins neuraminidase (NA) and 
haemagglutinin (HA), which play key roles in viral pathogenesis. Viral HA binds to sialic acid-containing receptors present on the surface of host cells, mediating attachment, fusion with host cell endosomes and subsequent endosomal membrane fusion releasing the viral genome into the cell ${ }^{86,87}$. In contrast, NA enzymatically cleaves sialic acid ${ }^{88}$ and the concerted activities of HA and NA are important in viral entry and release ${ }^{89,90}$. Influenza virus infects the human host by entry into the nasal passage where it replicates in mucosal epithelial cells, eventually spreading to the lower respiratory tract. The virus has a tropism for the respiratory tract, conferred by the preference for particular sialic acid linkages present on epithelial cells here ${ }^{91}$.

The burden of influenza can, in part, be attributed to the exceptional ability for the virus to evade host immune defences, largely through the ability to constantly undergo rapid genetic evolution. Genetic variability between seasonal influenza strains occurs through genetic drift, a process by which the highly error-prone viral RNA replication machinery rapidly generates genetic mutations, leading to the constant production of viral variants expressing proteins that are not recognised by the host's existing immune repertoire ${ }^{92}$. Coupled with selective pressure from the host immune system, mutants able to escape the host's existing strain-specific immune defences are selected for ${ }^{93}$. This results in a constantly evolving viral population that is able to successfully infect individuals with an existing immune response, allowing it to be passed through populations rapidly.

\subsubsection{Influenza vaccination}

Both natural infection and vaccination can confer resistance to influenza infection and severe disease. A major component of protection against influenza infection is antibody production, primarily to the viral surface proteins HA and $\mathrm{NA}^{94}$. Following natural infection, locally produced IgA antibody to influenza 
virus is secreted in the respiratory tract and is a correlate of protection against infection ${ }^{27,95}$. Secretory IgA neutralises influenza virus at the mucosa and is able to prevent the virus from crossing the mucosal barrier and entering the body ${ }^{27}$, as well as transporting virus that has invaded back out to the lumen ${ }^{96}$. Furthermore, infection elicits robust levels of virus-specific IgG antibody in the serum $^{97}$, which is a strong predictor of protection against influenza infection ${ }^{27,98,99}$. Influenza-specific CTL responses play a key role in limiting viral load and clearance of influenza infection, however do not appear to protect against initial infection ${ }^{100}$.

\subsubsection{Inactivated and live attenuated vaccines}

Currently, annual vaccination is widely considered the most effective method for preventing and controlling influenza virus infection ${ }^{101}$. International surveillance data from the World Health Organisation is used to inform the most prevalent circulating strains of each influenza season ${ }^{102,103}$ and currently licensed vaccines contain viral material derived from three to four of the most predominant strains, referred to as trivalent or quadrivalent, respectively ${ }^{103}$. Two major types of influenza vaccine are licensed for use: inactivated and live attenuated.

There are three types of inactivated vaccine, distinguished by the level of purification. Whole virus vaccines are simply composed of inactive whole virus $^{92}$. Split virus vaccines are further purified through addition of detergent to dissociate the viral lipid envelope, exposing viral proteins which are formulated into the vaccine ${ }^{104}$. For manufacture of subunit vaccines, viral components undergo further purification steps to isolate HA protein ${ }^{92}$. Due to the comparable immunogenicity of whole virus preparations, split and subunit vaccines and the lower reactogenicity of subunit vaccines ${ }^{105,106}$, these are the most widely utilised, in particular the trivalent influenza subunit vaccine (TIV). TIV is administered 
parenterally, either by the intramuscular or subcutaneous route. The major mechanism of protection by TIV is the induction of IgG antibody in the serum $^{107,108}$.

The other major licensed influenza vaccine in use is the live attenuated influenza vaccine (LAIV). LAIV is administered via an intranasal spray and contains an attenuated virus that is cold-adapted to only replicate efficiently in the nasopharynx, not the lung where pathology occurs ${ }^{109}$. The vaccine more closely resembles natural infection, eliciting protection by inducing both mucosal IgA secretion and systemic IgG antibody responses ${ }^{110-112}$.

\subsubsection{Immune response to influenza vaccines are suboptimal}

Despite widespread immunization against influenza, infection continues to cause severe illness and mortality worldwide. Current influenza vaccines currently are designed to induce primarily strain-specific immunity, and viral strains encountered may not match those included in vaccines ${ }^{113}$. Moreover, current influenza vaccines fail to protect against infection in many individuals even when the vaccine strains are well matched to circulating viruses. In individuals vaccinated with TIV and LAIV, vaccination protects against infection with matched strains in approximately $70 \%$ and $85 \%$ of people, respectively ${ }^{114}$. This reflects the high level of inter-individual variability in the strength of antibody responses to influenza vaccination, with antibody titres varying up to 100 -fold between individuals and many individuals do not achieve seroconversion ${ }^{115}$. Furthermore, particular populations mount poorer antibody responses to influenza vaccination, particularly the elderly ${ }^{116,117}$ and children and, importantly, infection has the greatest impact on these populations ${ }^{118}$. The factors leading to suboptimal antibody responses in these low responding groups are yet to be fully elucidated. 
It is understood that environmental factors are a key influence on human immune responses. A recent study compared an extensive panel of immune cells and soluble mediators between monozygous twins and found that non-heritable factors were the dominant determining factor of $77 \%$ of the immune parameters examined $^{119}$, therefore surpassing the importance of genetic contributions. Furthermore, it has also been found that cohabitating, yet unrelated, individuals show $50 \%$ less immunological variation than those of the general population ${ }^{120}$. These studies illustrate the need to elucidate the key environmental factors that influence the human immune system in order to determine interventions that will improve aberrant immune responses.

Although a number of factors have been identified to influence the efficacy of influenza vaccination, including age, immunocompetence, preexisting levels of antibody from previous infection and vaccination ${ }^{121}$, an emerging theory is that the microorganisms that inhabit the intestinal tract, the gut microbiota, may also be a contributing factor. Coinciding with similarities in immune profiles with unrelated housemates, cohabiting individuals show convergence in their gut microbial composition ${ }^{122,123}$. Furthermore, gut microbial composition of the elderly ${ }^{124}$ and young children ${ }^{125}$ differs significantly from adults, which may be a factor in poor vaccine responses in these groups. It has also been consistently found that vaccine efficacy is much poorer in developing nations, something that may be attributable to differences in gut microbiota composition ${ }^{126,127}$. There is now an accumulating body of direct evidence to support that gut microbial composition directly influences the immune response to vaccination. This will be expanded on in further chapters. 


\subsection{The gut microbiota}

Hippocrates was quoted as saying, "all disease begins in the gut" and over 2000 years later, the relevance of his sentiment is becoming apparent. The influence of the gut microbiota on human health has recently become of significant research interest with mounting evidence demonstrating the influence these microbes have on host wellbeing. The skin, respiratory, oral, urogenital and gastrointestinal tracts of mammals offer rich ecological niches that are constitutively colonised by unique communities of microorganisms ${ }^{128}$. The majority of these microorganisms are bacteria, with minor populations of fungi, archaea, protozoa and viruses, however for simplicity, in this thesis 'gut microbiota' will refer to only the bacterial communities. The gastrointestinal (GI) tract is home to the majority of these microbes, with the intestine of each human housing an estimated 39 trillion bacteria, likely exceeding the number of the individual's own cells ${ }^{129}$. The phyla Bacteroidetes and Firmicutes comprise more than $90 \%$ of the microbes of the human ${ }^{128}$ and murine ${ }^{130}$ gut microbiota. Other major phyla include Actinobacteria, Proteobacteria, Tenericutes and Verrucomicrobia, among others.

Gut microbes and their mammalian host share a symbiotic relationship, in which the host provides nutrition and favorable living conditions for microbes and, in turn, the microbiota aids in maintaining host health. The resident microbiota performs anaerobic fermentation of undigested dietary material and endogenous compounds, leading to production of a diverse collection of metabolites for use by the host ${ }^{131}$, as well as synthesising essential vitamins ${ }^{132}$. Additionally, the gut microbiota plays a key role in resistance to colonisation by enteric pathogens. It does so directly, by inhibiting pathogen growth through the secretion of anti-microbial molecules ${ }^{133}$ and limiting nutrient availability ${ }^{134}$, and indirectly promotes pathogen resistance by maintaining gut barrier function, for 
example by stimulating mucus secretion ${ }^{135}$. Vitally, the gut microbiota also provides signals for the development and proper function of the immune system $^{136}$, this will be expanded on in further chapters.

\subsubsection{Microbiota community analysis}

It is estimated that the genes of the human gut microbiota, the microbiome, contribute over 100 times the number of genes than the human genome ${ }^{137}$. However, each individual may only possess $15 \%$ of the full microbial compliment observed in humans ${ }^{138}$, illustrating the variability that exists between individuals. It is therefore essential to be able to identify and functionally assign the resident microbiota to elucidate key features that are important in health and disease in order to harness this knowledge to improve human health.

The development of next-generation sequencing technology has allowed the in-depth examination of the enormously complex commensal microbial communities, whether this is complete or targeted sequencing of DNA extracted from a sample, such as faeces. This has surpassed culture-dependent methods for bacterial identification, which limited analysis to the few organisms that can be grown under laboratory culture conditions ${ }^{139}$. Analysis initially focused on phylogenetic classification which provided insight into bacterial community members that are crucial to influencing host immunity. Phylogenetic classification is performed by sequencing the gene encoding $16 \mathrm{~S}$ ribosomal ribonucleic acid (rRNA), a prokaryotic gene that codes for part of the $30 \mathrm{~S}$ subunit of ribosomes. This gene is conserved within bacterial and archaeal species, allowing selective sequencing of the microbiome ${ }^{140}$ and importantly, it contains variable regions, allowing taxonomic classification of microbiota communities ${ }^{57}$. Quantitative polymerase-chain reaction (qPCR) with 16S rRNA-specific primers can also be used as a cost-effective alternative to classify microbial communities 
at the phyla level ${ }^{19}$. It is becoming apparent that the functional composition of the commensal microbiota is also likely to be of importance in influencing health, as many microbially-produced molecules are able to influence host homeostasis ${ }^{20}$. This can be explored using whole metagenomic sequencing of DNA extracted from a sample, allowing microbial genes to be annotated and catalogued into functional categories. This approach can be extremely useful to determine changes in microbial function relating to states of health or disease ${ }^{21}$.

However, often the genetic potential predicted from metagenomes does not necessarily equate to functional output ${ }^{20}$. Metatranscriptomics is also widely used in microbiota studies and quantifies transcriptional activity of microbial genes by sequencing messenger RNA (mRNA) transcripts ${ }^{22}$. Furthermore, translation of gut microbial genes can be quantified by metabolomics. This is often performed on faecal samples where a significant proportion of metabolites present will be microbially synthesised ${ }^{23}$. Each technique has advantages and these can be integrated to form a comprehensive picture of microbial community composition and function, especially when comparing the microbiota between states of health and disease ${ }^{141}$.

\subsubsection{The interface between the intestinal immune system and the microbiota}

The intestine is home to the largest number of immune cells in the body, which is unsurprising considering the antigenic load presented by symbiotic and pathogenic microbes, food and other exogenous materials that constantly occupy the intestinal lumen. The intestinal immune system is tasked with preventing translocation of intestinal contents into the sterile tissues and blood in order to curb infection, as well as preventing unnecessary inflammatory responses to innocuous antigens. Perhaps counterintuitively, the maintenance of intestinal homeostasis is reliant on the constant interactions between intestinal 
microbes and the mucosal immune system ${ }^{142}$. Germ-free (GF) mice are born and maintained in sterile isolators and a lack of all microbial stimuli leaves these mice highly susceptible to enteric infection compared to mice with a complete microbiota, known as specific-pathogen free $(\mathrm{SPF})$ mice $^{136}$. This is due to underdevelopment in the intestinal immune system. For example, the gut microbiota is crucial for proper maintenance of the intestinal mucus layer by goblet cells, providing a first-line defence against the microbiota and pathogens ${ }^{143}$. Gut microbes also promote the production of secretory $\operatorname{IgA}(\operatorname{sg} \mathrm{A})$, which binds to microbes in the intestine, protecting the epithelium from bacterial invasion and promoting tolerance to the microbiota ${ }^{144}$. Furthermore, specific members of gut microbiota promote homeostasis via stimulating development of specific $\mathrm{T}$ cell subsets. Segmented filamentous bacteria are able to promote TH17 skewing to protect against intestinal infection, while a community of Clostridia strains was shown to stimulate differentiation of $\mathrm{T}_{\mathrm{REGS}}{ }^{145}$. Mice with genetic defects in their ability to detect microbes, for example those lacking the bacterial sensor, TLR-4, or its downstream signalling molecule, MyD88, have worsened symptoms in a model of inflammatory bowel disease. Thus, it is clear that interactions between the microbiota and host immune system are crucial for maintenance of protection against local infection and inflammation.

\subsubsection{Gut microbiota interactions and the systemic immune system}

Intriguingly, microbiota effects extend beyond the local immune system of the intestine and it is clear that the gut microbiota provides diverse signals that are crucial for the development and function of host immunity at systemic sites. This is supported by observations that altered composition and function of the microbiota are prevalent in individuals with a number of immune-modulated diseases at sites distinct from the gut. For example, the development of allergic 
diseases in childhood is associated with reduced diversity of the intestinal microbiota in infants ${ }^{146}$. It has also been observed that microbial composition varies significantly between healthy individuals and those with type 1 diabetes ${ }^{147}$ and rheumatoid arthritis ${ }^{148}$. Furthermore, the composition of the infant gut microbiome has been shown to correlate with the strength of the antibody responses to rotavirus vaccination ${ }^{127}$. It is understood that the microbiota communicates with its host via two major molecular signals, either by translocation of components of microbial cells or via the action of microbially-produced metabolites.

\subsubsection{Microbial ligands}

As discussed earlier, PRRs on host immune cells play a key role in sensing microbial components in order to induce cellular effector mechanisms. Traditionally, it has been understood that intestinal lumen contents are compartmentalised, preventing translocation of microbes and their components into the sterile compartments of the body ${ }^{144}$. Mucus, epithelial cells and immune cells in the lamina propria as well as the liver maintain a barrier to minimise translocation to systemic circulation ${ }^{149}$, as breakdown in the barrier function can be detrimental and is linked to the pathogenesis of inflammatory bowel diseases $^{150}$, liver disease ${ }^{151}$ and sepsis ${ }^{152}$. However, more recently it has been proposed that this barrier is not absolute and microbial components are able to translocate across the mucosa into the circulation and influence immunity distal from the gut. This is supported by the presence of considerable titres of gut microbiota-specific IgG in the serum of healthy humans ${ }^{153}$ and mice ${ }^{154}$. Translocation of microbial cell components has been observed directly in healthy mice, whereby labelled peptidoglycan from the commensal bacterium Escherichia coli was shown to undergo constitutive translocation across the gut mucosa and bind the NLR, Nod1, on neutrophils in the BM, which directly enhanced their bacterial killing capacity ${ }^{155}$. 
A number of other investigations have shown the requirement for sensing of bacterial components via PRRs in the development of robust immune responses distal from the gut. GF mice exhibit poor control of pulmonary infection with Klebsiella pneumoniae compared to SPF mice ${ }^{156}$. Injection with the bacterial membrane component, lipopolysaccharide (LPS), prior to infection enhances neutrophil recruitment, chemokine and cytokine production in the lung, and restores control of infection to levels of SPF mice ${ }^{156}$. Similarly, antibiotic treatment of SPF mice leads to defective T and B cell responses to influenza virus infection which could be restored by intrarectal administration of TLR agonists, mimicking the presence of bacteria in the colon ${ }^{157}$. In the context of influenza vaccination, it has been found that signalling driven by microbiota-derived flagellin via TLR-5 on B cells and macrophages was required for robust IgG antibody responses to TIV vaccination ${ }^{158}$. Accordingly, antibiotic-treated mice exhibited poor antibody responses to TIV, however this could be rescued by the simultaneous administration of flagellin ${ }^{158}$. Sensing of microbiota components via TLRs has also been shown to be crucial for haematopoesis. GF mice have a reduced pool of myeloid precursors and mature myeloid cells in the BM, resulting in significantly reduced frequencies of peripheral leukocytes and impaired control of systemic Escherichia coli infection ${ }^{159}$. Strikingly, the BM myeloid compartment in GF mice could be restored by transferring heat-treated serum from SPF animals, an effect that was dependent on TLR signalling ${ }^{159}$. Together these investigations show that sensing of systemic microbial components is required for the function of multiple immune cell types to respond effectively to infection and vaccination.

\subsubsection{Microbial metabolites}

The gut microbiota produces a complex collection of molecules, many of which have been shown to influence host immunity, capable of both enhancing and dampening immune responses depending on the molecule and their 
physiological context. The microbiota possesses a number of digestive and metabolic enzymes that metabolise dietary components that are otherwise indigestible to the host, as well as endogenous compounds that are produced by the host or the microbiota itself. This process results in the production of a diverse collection of metabolites in the gut, many of which are absorbed through the gut mucosa and exert effects on host cells locally or systemically ${ }^{160}$. Advances in characterisation of microbial metabolism through metagenomic and metabolomic analysis have identified microbial-produced molecules that have profound effects on the host immune system. The potential for small molecules to be absorbed into the circulation means they can act distally from the gut and therefore exert widespread influences on systemic immunity.

The most extensively studied of the microbiota metabolites are short-chain fatty acids (SCFAs). SCFAs are the major product of the anaerobic fermentation of digestion-resistant starches and soluble dietary fibre, known as microbiota-accessible carbohydrates (MACs) by certain bacteria in the colon ${ }^{161}$, explaining the very low levels of SCFA in the gut of GF mice ${ }^{162}$. The beneficial effects of dietary fibre and consequent SCFA production in disease prevention have been demonstrated in allergic airway inflammation ${ }^{163}$, obesity ${ }^{164}$ and anti-tumour immunity ${ }^{165}$. Coincident with increases in these and other diseases linked with SCFAs is systematic changes in human diet, notably the reduced intake of dietary fibre ${ }^{166}$. The three major SCFAs in the colon of humans and mice are acetate, butyrate and propionate ${ }^{167}$, the majority of which are absorbed by the host or further utilised by commensal bacteria, with only $5-10 \%$ excreted in faeces ${ }^{168}$. Beyond their use in the gut, SCFAs are easily detectable in the peripheral circulation ${ }^{167}$. Here, they are available for use in tissues and have been shown to exhibit systemic immunomodulatory effects in the host via a number of mechanisms. SCFAs signal via the G-protein coupled receptors (GPCRs) GPR41, GPR43 and GPR109A with varying affinities and SCFA preference ${ }^{169,170}$. 
These receptors are widely expressed by immune cells, including DCs, monocytes, $\mathrm{M} \varphi$, as well as in the $\mathrm{BM}^{169,171}$. The pervasive immunomodulatory effect of SCFAs on host immunity is demonstrated in mice deficient in GPR43 $\left(\right.$ GPR43 $\left.{ }^{-1}\right)$, for which the only known ligands are SCFAs. These mice exhibit exacerbated inflammatory responses in models of arthritis, allergic airway inflammation and colitis ${ }^{172}$. SCFAs also promote intestinal homeostasis by providing a primary energy source for colonic epithelial cells to support integrity of the intestinal barrier ${ }^{173,174}$. Beyond the gut, SCFAs are taken up by B cells ${ }^{175}$ and $\mathrm{CD}^{+}$memory $\mathrm{T}$ cells ${ }^{176}$ and converted to acetyl CoA, fueling metabolism to drive both antibody production ${ }^{175}$ and function of memory CD8 ${ }^{+}$T cells ${ }^{176}$. SCFAs also influence the host epigenome by inhibiting histone deacetylases (HDACs) ${ }^{177,178}$. HDACs induce transcriptional repression by the removal of acetyl groups from lysine residues within histones, reducing transcription enzyme accessibility to promoter regions in the DNA. Therefore, HDAC inhibitors increase gene transcription ${ }^{179}$. Oral administration of SCFAs inhibits HDACs to regulate the mTOR-S6K pathway in T cells, which is important for their differentiation, promoting a $\mathrm{T}_{\mathrm{H}} 1$ and $\mathrm{T}_{\mathrm{H}} 17$ phenotype in peripheral $\mathrm{T}$ cells to enhance protection during systemic bacterial infection ${ }^{180}$. Butyrate is a particularly potent HDAC inhibitor and promotes peripheral TREG generation, both by directly enhancing acetylation of the TREG master transcription factor locus, FoxP3, as well as reducing DC expression of pro-inflammatory cytokines ${ }^{181}$.

Numerous other microbial metabolites have been identified to be important for immune development and fine tuning of immune responses ${ }^{131}$. For example, the microbiota is able to synthesise a variety of polyamines which have been shown to be able to modulate systemic immune responses, including enhancing splenic B cell maturation ${ }^{182}$. Based upon the observation that the gut microbiota is able to regulate immune responses to viral infection by modulating type I interferon (IFN) signalling and robust type I IFN response is critical in anti-viral immune 
responses ${ }^{183}$, Steed and colleagues screened microbial metabolites for their ability to induce type I IFN signalling ${ }^{184}$. They identified a metabolite from dietary flavonoids, desaminotyrosine, that potently enhanced signalling and subsequently promoted resistance to influenza infection ${ }^{184}$. These compounds are some examples of the vast collection of metabolites produced by the gut microbiota, many of which have potent immunomodulatory properties.

\subsubsection{Dysbiosis}

It is clear that the gut microbiota exerts widespread influence on many aspects of host physiology, including to enhancing antibody responses to influenza vaccination, which is the focus of this investigation. It has been proposed that recent changes in the lifestyle and environmental of those in Western societies is significantly altering gut microbiota communities, a state termed dysbiosis, and this may be predisposing humans to disease ${ }^{185}$.

Changes in the environment can rapidly change the composition and function of gut microbial communities and, in turn, alter essential interactions with the host. Antibiotics are a major bacterial perturbation introduced to humans in the last century and are routinely prescribed, with children in the US receiving an average of ten courses before they reach ten years old ${ }^{186}$. Of concern, antibiotic use in infants leads to alterations in gut microbiota composition ${ }^{187}$ and administration of antibiotics to healthy adults for only one week led to significant changes to gut microbiota communities ${ }^{188}$. In both these studies, alterations were shown to persist beyond two years after treatment.

Diet also has a profound influence on gut microbiota structure and function. In two controlled feeding studies in humans, it has been observed that after just 24 hours of changes in the diet, significant alterations occur to composition of the gut microbiota ${ }^{189,190}$. The Western diet is dominated by refined carbohydrates, fat 
and sugar and typically contains dietary additives and low amounts of fibre ${ }^{191}$, in contrast to that of those living traditional lifestyles, such as hunter-gatherer societies ${ }^{192}$. Coinciding with dietary changes are significant differences between the microbiome of Westerners and of hunter-gatherers, including reduced diversity and an altered ratio of the major bacterial phyla, Bacteroidetes and Firmicutes ${ }^{192}$. Dietary impacts on the microbiota have been directly demonstrated in mice, for instance, reducing fibre intake severely reduces gut microbial diversity ${ }^{193}$, as well as dietary additives, such as emulsifiers ${ }^{194}$ and artificial sweeteners ${ }^{195}$, significantly shifting the composition of microbial communities.

These changes in microbial composition through lifestyle changes are likely contributing to the rise in immunological diseases. In a number of disease states, shared microbiota characteristics within diseased populations have been determined. For example, loss of diversity of faecal microbiota communities have been found in individuals with Crohn's disease ${ }^{196}$ and type 1 diabetes ${ }^{197}$. Furthermore, antibiotic use in infants has been strongly linked to the development of asthma and allergy in later childhood ${ }^{187,198}$. In addition, poor antibody responses to the rotavirus vaccine in infants was associated with increased Bacteroidetes and lower abundance of Streptococcus bovis ${ }^{127}$. However, due to uniqueness of each individual's gut microbial community, it can be difficult to define what constitutes a health- or disease-associated microbiota. It is crucial that common features of dysbiosis are identified and their involvement in pathogenesis of disease elucidated, allowing for the rational design of microbiota-altering therapeutic approaches.

\subsubsection{Beneficial modulation of the gut microbiota}

While the plasticity of the microbiota can result in maladaptive changes, it also presents the exciting possibility of therapeutic modulation of the microbiota for 
disease prevention or cure. Diet is considered the most affordable, safe and accessible potential therapy for modulating the gut microbiota. Diet rapidly alters gut microbiota, with a change in diet significantly altering the community composition in as little as one day ${ }^{190,199}$. It has also been observed that alterations to the diet are able to reproducibly alter gut microbiota composition of different mouse strains mutant for genes important for host-microbial interactions ${ }^{199}$, demonstrating that diet is strong defining factor of microbiota composition that may overshadow even genetic differences between individuals.

Given the role of the microbiota in shaping innate and adaptive immunity, a number of studies have investigated the therapeutic potential of modulation of the gut microbiota in improving immunity in infection and vaccination. A common approach used to modify the gut microbiota is the use of probiotic supplementation, defined as live microorganisms that when administered confer a health benefit to the host ${ }^{200}$. Additionally, MACs that selectively change the structure and function of gut microbial communities have been trialed for therapeutic use - these are referred to as prebiotics ${ }^{201}$. Major investigations of the therapeutic benefits of probiotic and prebiotic foods in influenza infection and vaccination are summarised below.

\subsubsection{Probiotics}

Probiotics may elicit immunomodulatory effects via direct recognition of microbial molecules by the host immune system through PRRs or by modulating composition of the endogenous microbiota ${ }^{202}$ and in this way have the potential to exert systemic influences on the host immune response.

Administration of probiotics to enhance protection in influenza infection and vaccination have shown some promise. Efficacy of probiotics in infection are limited to mice. It was demonstrated that administration of Lactobacillus gasseri promoted antiviral immune responses in the lung tissue following influenza 
infection, whilst limiting excessive inflammatory cell infiltration and cytokine production in the lung ${ }^{203}$. L. casei administration was shown to improve control of viral load by enhancing production of inflammatory cytokines by lymphocytes in the respiratory tract ${ }^{204}$. In human trials, some benefit has been demonstrated with probiotic supplementation in conjunction with influenza vaccines. Benefit was observed following oral intake of $L$. casei and Bifidobacterium animalis in healthy adults, where administration of both probiotic strains resulted in increased vaccine-specific total IgG, IgG1 and IgG3 titres compared to placebo ${ }^{205}$. Furthermore, administration of L. rhamnosus GG prior to LAIV vaccination in healthy adults was shown to significantly enhance production of vaccine-specific neutralising antibody in the serum ${ }^{206}$. L. fermentum intake was shown to significantly enhanced the production of vaccine-specific IgA in the serum following TIV, however IgG was unchanged between probiotic and placebo groups ${ }^{207}$. A number of studies have also been specifically conducted in the elderly. Oral intake of L. casei in an elderly population significantly enhanced influenza B virus-specific antibody titres following TIV 208 . Another study found that administration of $L$. paracasei with a prebiotic fructo-oligosaccharide to the elderly showed no change in antibody production in response to $\mathrm{TIV}^{209}$. These studies demonstrate that probiotic supplementation shows promise in enhancing immunity in influenza infection and vaccination, however efficacy is hard to interpret as differences in strains and dosages vary significantly between studies and a strain-specific approach is clearly required.

\subsubsection{Prebiotics}

Prebiotics have the potential to significantly alter microbiota-host interactions to influence health of the host. This may be mediated by increasing the availability of substrates for microbial metabolism. For example, increased fibre intake provides fermentable polysaccharides as substrates for metabolism, therefore increasing SCFA levels in the colon and blood ${ }^{163,175,192}$. Additionally, prebiotics 
may encourage the growth of specific species and alter gut microbiota community structure. In areas where fibre intake is high, there is increased proportions of SCFA-producing bacteria in the gut ${ }^{192}$. Therefore, prebiotic intake may alter both the gut microbial metabolites and the community composition, and therefore microbial ligands, which have the potential to have systemic effects on host immunity.

The number of studies investigating prebiotics alone on the immune response to influenza vaccination in human trials are limited and results have been mixed. One study found that administration of a prebiotic mixture of $\beta 2-1$ fructans, inulin and oligofructose, to a healthy cohort of adults prior to TIV significantly enhanced the vaccine-induced IgG1 antibody response ${ }^{210}$. Administration of an oligosaccharide mixture in an elderly cohort prior to influenza vaccination had no effect on vaccine-specific antibody in the serum ${ }^{211}$. Again, the type of prebiotic, dosages and duration of treatment vary widely between studies and these factors are likely to be crucial to their efficacy. It should also be noted that, unlike probiotics where effects of administration are undoubtedly microbiota-dependent, difficulty can arise in determining if a prebiotic is exerting immunomodulatory effects via the microbiota, as foods may be affecting immune pathways in other ways.

As many foods contain MACs, notably vegetables and fruits, they have prebiotic potential and may be 'functional foods': foods that deliver health benefits additional to providing basic nutrition. Further research into the potential benefit of prebiotic functional foods in immune responses to influenza vaccination are required. 


\subsection{Aims and hypotheses}

Whilst vaccination protects against influenza infection in much of the population, a significant proportion of individuals fail to elicit sufficient protective antibody responses and a strong need remains to improve the efficacy of these vaccines. The literature presented so far demonstrates that the gut microbiota exerts an influence over many facets of the host immune system, not only in promoting homeostasis locally, but also having a pervasive effect on sites distal from the intestine. Recent investigations have identified pathways influenced by the gut microbiota to promote protective responses to both influenza infection ${ }^{157,212}$ and vaccination ${ }^{158}$, however it is clear that the microbiota works in a multitude of ways. The first aim of this investigation was to establish an antibiotic model in which vaccine-specific antibody production was rendered defective and subsequently use this model to investigate what immune cell processes to influenza vaccination are impacted by manipulating the gut microbiota.

Furthermore, because the gut microbiota is able to significantly influence immune responses, this opens up avenues for microbiota-altering therapies for treating immune-modulated disorders and enhancing vaccine responsiveness. However, evidence for the efficacy of specific foods in altering the microbiota and enhancing the immune response to influenza vaccination are lacking. Therefore, my second aim was to test the efficacy of a number of microbiota-altering nutritional interventions to improve the immune response to influenza vaccination. 
Specific aims:

1) To develop a model of oral antibiotic administration that negatively affects antibody responses to TIV vaccination.

2) To assess changes in immune cell frequency and function in the humoral immune response to TIV vaccination following microbiota alteration with antibiotic treatment.

3) To establish mouse models of parenteral and mucosal influenza vaccination that model low-responding human populations, characterised by suboptimal antibody production and protection against subsequent infection.

4) To assess the capacity of orally administered nutritional interventions with microbiota-altering potential to improve suboptimal immune responses in the influenza vaccination model. 

2 Materials and Methods 


\subsection{Materials}

\subsubsection{Labware}

Table 2.1: List of labware

\begin{tabular}{|l|l|}
\hline Product & Supplier \\
\hline 22-gauge gavage needle & $\begin{array}{l}\text { Total Lab Systems, Auckland, New } \\
\text { Zealand }\end{array}$ \\
\hline $\begin{array}{l}\text { Axygen filtered, sterile, RNase free } \\
\text { pipette tips - } 10 \mu \mathrm{L}, 20 \mu \mathrm{L}, 200 \mu \mathrm{L} \\
\text { and } 1000 \mu \mathrm{L}\end{array}$ & $\begin{array}{l}\text { Axygen Scientific, California, United } \\
\text { States }\end{array}$ \\
\hline $\begin{array}{l}\text { Axygen microtubes }-0.6 \mathrm{~mL}, 1.7 \\
\mathrm{~mL} \text { and } 2.0 \mathrm{~mL}\end{array}$ & \\
\hline $\begin{array}{l}\text { Axygen pipette tips }-10 \mu \mathrm{L}, 20 \mu \mathrm{L}, \\
\text { 200 } \mu \mathrm{L} \text { and } 1000 \mu \mathrm{L}\end{array}$ & \\
\hline $\begin{array}{l}\text { BD } 5 \mathrm{~mL} \text { polystyrene } \\
\text { round-bottom tubes }\end{array}$ & ZD Biosciences, Auckland, New \\
\hline $\begin{array}{l}\text { BD Falcon } 70 \mu \mathrm{m} \text { cell strainer } \\
\text { BD Falcon cell scrapers, } 25 \mathrm{~cm} \\
\text { handle and } 1.8 \mathrm{~cm} \text { blade }\end{array}$ & \\
\hline $\begin{array}{l}\text { BD Falcon polystyrene conical } \\
\text { tubes - } 15 \mathrm{~mL}, 50 \mathrm{~mL} \\
\text { BD Falcon polystyrene flat-bottom } \\
\text { plates - } 96 \text {-well, } 24 \text {-well } \\
\text { BD Falcon polystyrene } \\
\text { round-bottom plates - } 96-w e l l\end{array}$ \\
\hline
\end{tabular}




\begin{tabular}{|c|c|}
\hline $\begin{array}{l}\text { BD Falcon serological pipettes }-2 \\
\mathrm{~mL}, 5 \mathrm{~mL}, 10 \mathrm{~mL} \text { and } 25 \mathrm{~mL}\end{array}$ & \\
\hline $\begin{array}{l}\text { BD PrecisionGlide needles - } 18 \\
\text { gauge } 40 \mathrm{~mm} \text {, } 25 \text { gauge } 40 \mathrm{~mm}\end{array}$ & \\
\hline BD syringes - $1 \mathrm{~mL}, 2 \mathrm{~mL}$ & \\
\hline $\begin{array}{l}\text { BioRad multiplate 96-well PCR } \\
\text { plates, unskirted, clear }\end{array}$ & BioRad, Auckland, New Zealand \\
\hline $\begin{array}{l}\text { Corning polystyrene v-bottom } \\
\text { plates - 96-well }\end{array}$ & $\begin{array}{l}\text { Sigma Aldrich, Auckland, New } \\
\text { Zealand }\end{array}$ \\
\hline $\begin{array}{l}\text { Disposable carbon steel surgical } \\
\text { blades }\end{array}$ & $\begin{array}{l}\text { Swann-Morton, Sheffield, United } \\
\text { Kingdom }\end{array}$ \\
\hline $\begin{array}{l}\text { Eppendorf safe-lock } \\
\text { microcentrifuge tubes - } 2 \mathrm{ml}\end{array}$ & $\begin{array}{l}\text { Sigma Aldrich, Auckland, New } \\
\text { Zealand }\end{array}$ \\
\hline Interlab sealplate classic films & Interlab, Wellington, New Zealand \\
\hline MACS SmartStrainer $70 \mu \mathrm{M}$ & Pharmaco, Auckland, New Zealand \\
\hline $\begin{array}{l}\text { MicroAmp } 0.1 \mathrm{~mL} \text { fast optical 96- } \\
\text { well reaction plate with barcode }\end{array}$ & $\begin{array}{l}\text { Thermofisher Scientific, Auckland, } \\
\text { New Zealand }\end{array}$ \\
\hline MicroAmp optical adhesive film & \\
\hline Nalgene cryogenic vials $-2 \mathrm{~mL}$ & $\begin{array}{l}\text { Sigma Aldrich, Auckland, New } \\
\text { Zealand }\end{array}$ \\
\hline Nunc 96-well Maxisorb plates & $\begin{array}{l}\text { Thermofisher Scientific, Auckland, } \\
\text { New Zealand }\end{array}$ \\
\hline Qiagen $5 \mathrm{~mm}$ stainless steel beads & Bio-Strategy, Victoria, Australia \\
\hline $\begin{array}{l}\text { Sarstedt Microvette } 500 \text { serum gel } \\
\text { tubes }\end{array}$ & $\begin{array}{l}\text { Global Science and Technology Ltd, } \\
\text { Auckland, New Zealand }\end{array}$ \\
\hline
\end{tabular}




\begin{tabular}{|l|l|}
\hline $\begin{array}{l}\text { Satorius Vivaspin 20 10,000 } \\
\text { MWCO column }\end{array}$ & $\begin{array}{l}\text { MicroNZ, Martinborough, New } \\
\text { Zealand }\end{array}$ \\
\hline $\begin{array}{l}\text { Slide-A-Lyzer 10,000 MWCO } \\
\text { dialysis cassettes }\end{array}$ & $\begin{array}{l}\text { Thermofisher Scientific, Auckland, } \\
\text { New Zealand }\end{array}$ \\
\cline { 1 - 2 } Sterilin 139 mm sterile petri dishes & \\
\hline
\end{tabular}

\subsubsection{Reagents}

Table 2.2: List of reagents

\begin{tabular}{|c|c|}
\hline Product & Supplier \\
\hline Ambion DEPC-treated $\mathrm{H}_{2} \mathrm{O}$ & \multirow[t]{2}{*}{$\begin{array}{l}\text { Thermofisher Scientific, Auckland, } \\
\text { New Zealand }\end{array}$} \\
\hline $\begin{array}{l}\text { Ambion ethylenediaminetetraecetic } \\
\text { acid (EDTA) }\end{array}$ & \\
\hline Ampicillin sodium salt & $\begin{array}{l}\text { Sigma Aldrich, Auckland, New } \\
\text { Zealand }\end{array}$ \\
\hline $\begin{array}{l}\text { Applied Biosystems SYBR Green } \\
\text { PCR Master Mix }\end{array}$ & \multirow[t]{2}{*}{$\begin{array}{l}\text { Thermofisher Scientific, Auckland, } \\
\text { New Zealand }\end{array}$} \\
\hline $\begin{array}{l}\text { Applied Biosystems TaqMan Fast } \\
\text { Advanced Master Mix }\end{array}$ & \\
\hline $\begin{array}{l}\text { BD compensation beads anti-rat } \\
\text { anti-hamster }\end{array}$ & $\begin{array}{l}\text { BD Biosciences, Auckland, New } \\
\text { Zealand }\end{array}$ \\
\hline
\end{tabular}




\begin{tabular}{|c|c|}
\hline $\begin{array}{l}\text { Bifidobacterium animalis subsp. lactis } \\
\text { lyophilised powder }\end{array}$ & Fonterra, Auckland, New Zealand \\
\hline Chloroform & $\begin{array}{l}\text { Sigma Aldrich, Auckland, New } \\
\text { Zealand }\end{array}$ \\
\hline $\begin{array}{l}\text { Dulbecco's phosphate buffered } \\
\text { saline (PBS) powder }\end{array}$ & $\begin{array}{l}\text { Thermofisher Scientific, Auckland, } \\
\text { New Zealand }\end{array}$ \\
\hline Ethanol $(100 \%)$ & Pure Science, Porirua, New Zealand \\
\hline Fresh Zespri Sungold kiwifruit & $\begin{array}{l}\text { Zespri, Mount Maunganui, New } \\
\text { Zealand }\end{array}$ \\
\hline Fructose & $\begin{array}{l}\text { Sigma Aldrich, Auckland, New } \\
\text { Zealand }\end{array}$ \\
\hline $\begin{array}{l}\text { Gibco Dulbecco's Modified Eagle } \\
\text { Medium (DMEM) }\end{array}$ & $\begin{array}{l}\text { Thermofisher Scientific, Auckland, } \\
\text { New Zealand }\end{array}$ \\
\hline Gibco foetal bovine serum (FBS) & \\
\hline $\begin{array}{l}\text { Gibco Iscove's Modified Dulbecco's } \\
\text { Medium (IMDM) }\end{array}$ & \\
\hline $\begin{array}{l}\text { Gibco sterile phosphate-buffered } \\
\text { saline (PBS) }\end{array}$ & \\
\hline Gibco trypan blue $(0.4 \%)$ & \\
\hline
\end{tabular}




\begin{tabular}{|c|c|}
\hline Glucose & $\begin{array}{l}\text { Sigma Aldrich, Auckland, New } \\
\text { Zealand }\end{array}$ \\
\hline Hansell's strawberry essence & $\begin{array}{l}\text { Countdown, Wellington, New } \\
\text { Zealand }\end{array}$ \\
\hline High Capacity RNA to cDNA kit & $\begin{array}{l}\text { Thermofisher Scientific, Auckland, } \\
\text { New Zealand }\end{array}$ \\
\hline $\begin{array}{l}\text { HRP-conjugated anti-mouse IgG } \\
\text { secondary antibody }\end{array}$ & \\
\hline $\begin{array}{l}\text { HRP-conjugated anti-mouse IgG1 } \\
\text { secondary antibody }\end{array}$ & \\
\hline $\begin{array}{l}\text { HRP-conjugated anti-mouse IgA } \\
\text { secondary antibody }\end{array}$ & Southern Biotech, Birmingham, USA \\
\hline HKx31 influenza A virus & $\begin{array}{l}\text { Gifted from Lisa Hyland and Sam } \\
\text { Hou, Otago University }\end{array}$ \\
\hline Indian Ink & $\begin{array}{l}\text { Whitcoulls, Wellington, New } \\
\text { Zealand }\end{array}$ \\
\hline $\begin{array}{l}\text { Influvac }^{\circledR} 2016 \text { Inactivated TIV } \\
\text { Vaccine }\end{array}$ & Mylan, Auckland, New Zealand \\
\hline $\begin{array}{l}\text { Invitrogen OneComp eBeads } \\
\text { Compensation Beads }\end{array}$ & $\begin{array}{l}\text { Thermofisher Scientific, Auckland, } \\
\text { New Zealand }\end{array}$ \\
\hline Invitrogen Trizol Reagent & \\
\hline
\end{tabular}




\begin{tabular}{|c|c|}
\hline Isopropanol & $\begin{array}{l}\text { Sigma Aldrich, Auckland, New } \\
\text { Zealand }\end{array}$ \\
\hline Metronidazole & \\
\hline Mouse IgA Ready-SET-Go ELISA kit & $\begin{array}{l}\text { Thermofisher Scientific, Auckland, } \\
\text { New Zealand }\end{array}$ \\
\hline Mylan Vancomycin & $\begin{array}{l}\text { Pharmacy Department, Wellington } \\
\text { Hospital, New Zealand }\end{array}$ \\
\hline Neomycin trisulfate & $\begin{array}{l}\text { Sigma Aldrich, Auckland, New } \\
\text { Zealand }\end{array}$ \\
\hline $\begin{array}{l}\text { Pierce Bicinchoninic Acid Protein } \\
\text { Assay (BCA assay) Kit }\end{array}$ & $\begin{array}{l}\text { Thermofisher Scientific, Auckland, } \\
\text { New Zealand }\end{array}$ \\
\hline $\begin{array}{l}\text { Propolis formulated with } \gamma \text { - } \\
\text { cyclodextrin (Cyclopower) powder }\end{array}$ & $\begin{array}{l}\text { Manuka Health, Te Awamutu, New } \\
\text { Zealand }\end{array}$ \\
\hline QIAamp Fast DNA Stool Mini Kit & $\begin{array}{l}\text { BioStrategy, Auckland, New } \\
\text { Zealand }\end{array}$ \\
\hline DNase I & Roche, Mannheim, Germany \\
\hline Liberase TL & \\
\hline Sodium acetate & $\begin{array}{l}\text { Sigma Aldrich, Auckland, New } \\
\text { Zealand }\end{array}$ \\
\hline Sodium butyrate & \\
\hline
\end{tabular}




\begin{tabular}{|l|l|}
\hline Sodium propionate & \\
\hline Splenda artificial sweetener & $\begin{array}{l}\text { Countdown, Wellington, New } \\
\text { Zealand }\end{array}$ \\
\hline Sucrose & $\begin{array}{l}\text { Sigma Aldrich, Auckland, New } \\
\text { Zealand }\end{array}$ \\
\hline
\end{tabular}

\subsubsection{Buffers and reagents}

Flow buffer

FBS was added to PBS to give a final concentration of $2 \%$ FBS.

Anaesthetic working solution

A 10x stock solution of ketamine/xylazine anaesthetic solution was provided by the Biomedical Research Unit (BRU) at the Malaghan Institute of Medical Research (MIMR (Wellington, New Zealand)) at a concentration of $87 \mathrm{mg} / \mathrm{mL}$ of ketamine and $2.6 \mathrm{mg} / \mathrm{mL}$ of xylazine. This was diluted 1:10 in sterile PBS for a $1 \mathrm{x}$ working solution for intraperitoneal administration.

Lymph node digestion mix

IMDM was supplemented with $1 \mu \mathrm{g} / \mathrm{mL}$ liberase TL and $100 \mu \mathrm{g} / \mathrm{mL}$ DNase I. 


\subsubsection{Flow cytometry antibodies}

Table 2.3: List of flow cytometry antibodies

\begin{tabular}{|c|c|c|c|c|}
\hline Specificity & Fluorophore & Clone & Supplier & $\begin{array}{c}\text { Catalogue } \\
\#\end{array}$ \\
\hline B220/CD45R & $\begin{array}{l}\text { PE-CF594 } \\
\text { BV786 }\end{array}$ & $\begin{array}{l}\text { RA3-6B2 } \\
\text { RA3-6B2 }\end{array}$ & $\begin{array}{l}\text { BioLegend } \\
\text { BD Biosciences }\end{array}$ & $\begin{array}{l}103254 \\
563894\end{array}$ \\
\hline CD11b & BUV737 & $\mathrm{M} 1 / 70$ & BD Biosciences & 564443 \\
\hline CD11c & PECy7 & HL3 & BD Biosciences & 558079 \\
\hline CD138 & $\begin{array}{l}\text { BUV737 } \\
\text { PE }\end{array}$ & $\begin{array}{l}281-2 \\
281-2\end{array}$ & $\begin{array}{l}\text { BD Biosciences } \\
\text { BD Biosciences }\end{array}$ & $\begin{array}{l}564430 \\
553714\end{array}$ \\
\hline $\mathrm{CD} 3$ & $\mathrm{PE}$ & $145-2 \mathrm{C} 11$ & $\begin{array}{l}\text { Thermofisher } \\
\text { Scientific }\end{array}$ & $12-0031-82$ \\
\hline $\begin{array}{l}\text { CD32/16 } \\
\text { (Fc } \gamma \text { RII/III) }\end{array}$ & - & $2.4 \mathrm{G} 2$ & MIMR & - \\
\hline CD326 & BV711 & G8.8 & BD Biosciences & 563134 \\
\hline $\mathrm{CD} 4$ & BUV805 & GK1.5 & BD Biosciences & 564922 \\
\hline CD86 & BUV395 & GL1 & BD Biosciences & 564199 \\
\hline
\end{tabular}




\begin{tabular}{|c|c|c|c|c|}
\hline CXCR5 & Biotin & $2 \mathrm{G} 8$ & BD Biosciences & 551960 \\
\hline $\mathrm{F} 4 / 80$ & $\begin{array}{l}\text { Biotin } \\
\text { FITC }\end{array}$ & $\begin{array}{l}\text { BM8 } \\
\text { NAWESLEE }\end{array}$ & $\begin{array}{l}\text { BioLegend } \\
\text { Thermofisher } \\
\text { Scientific }\end{array}$ & $\begin{array}{l}123105 \\
13-7691-82\end{array}$ \\
\hline GL7 & FITC & GL7 & BD Biosciences & 553666 \\
\hline $\operatorname{IgD}$ & APC-H7 & IA6-2 & BD Biosciences & 561305 \\
\hline IgG1 & BV421 & A85-1 & BD Biosciences & 562580 \\
\hline $\operatorname{IgM}$ & APC & $\mathrm{II} / 41$ & BD Biosciences & 550676 \\
\hline Ly6C & Pacific blue & HK1.4 & BioLegend & 128013 \\
\hline $\begin{array}{l}\text { MHC II } \\
\text { (I-A/I-E) }\end{array}$ & AF647 & M5/114.15.2 & BioLegend & 107618 \\
\hline Streptavidin & $\begin{array}{l}\text { BV605 } \\
\text { BUV395 }\end{array}$ & - & $\begin{array}{l}\text { BD Biosciences } \\
\text { BD Biosciences }\end{array}$ & $\begin{array}{l}563260 \\
564176\end{array}$ \\
\hline
\end{tabular}




\subsubsection{Flow cytometry viability dyes}

Table 2.4: List of flow cytometry viability dyes

\begin{tabular}{|l|l|}
\hline \multicolumn{1}{|c|}{ Dye } & \multicolumn{1}{c|}{ Supplier } \\
\hline $\begin{array}{l}\text { 4',6-diamidino-2-phenylindole } \\
\text { (DAPI) }\end{array}$ & $\begin{array}{l}\text { Thermofisher Scientific, Auckland, } \\
\text { New Zealand }\end{array}$ \\
\cline { 1 - 1 } Live/dead fixable blue dead cell dye & \\
\cline { 1 - 2 } Zombie NIR fixable viability dye & BioLegend, Auckland, New Zealand \\
\hline
\end{tabular}

\subsubsection{Mice}

\subsubsection{Animal husbandry and ethics}

Mice were bred and housed within the BRU at MIMR in specific-pathogen free (SPF) conditions. Unless specified, mice were maintained on autoclaved meatfree rat and mouse diet (Specialty Feeds, Western Australia) and autoclaved acidified water. The Victoria University Animal Ethics Committee granted ethical approval for experimental animal procedures and all experiments were carried out in accordance with their guidelines. Mice were treated under the animal ethics protocols 22735 and 2014R16M.

\subsubsection{Mouse strains}

C57BL/6 mice were originally sourced from the Jackson laboratory (Bar Harbor, ME, USA), and bred at the MIMR BRU. 


\subsection{Methods}

\subsubsection{Mouse manipulations}

\subsubsection{Broad-spectrum antibiotic cocktail administration}

Broad-spectrum antibiotic cocktail (ABX) consisted of vancomycin at $0.5 \mathrm{mg} / \mathrm{mL}$, neomycin at $1 \mathrm{mg} / \mathrm{mL}$, ampicillin at $1 \mathrm{mg} / \mathrm{mL}$ and metronidazole at $1 \mathrm{mg} / \mathrm{mL}$, dissolved in purified, acidified drinking water provided by the MIMR BRU. This was administered to mice ad libitum for the amount of time specified in each experiment. Splenda artificial sweetener $(4 \mathrm{mg} / \mathrm{mL})$ and Hansell's strawberry essence $(2 \mu \mathrm{L} / \mathrm{mL})$ were added to improve palatability of the solution.

\subsubsection{Trivalent influenza vaccine (TIV) administration}

Influvac ${ }^{\circledR} 2016$ inactivated TIV vaccine was diluted 1:4 in sterile PBS and $200 \mu \mathrm{L}$ was administered subcutaneously (SC) at the tail base between the dorsal and right lateral vein, with mice receiving $1 / 10$ of the full human dose of $500 \mu \mathrm{L}$.

\subsubsection{Inactivated $\mathrm{HK} \times 31$ virus vaccine administration}

Inactivated $\mathrm{HK} \times 31$ vaccines were diluted in sterile PBS at specified concentrations. For SC vaccination, $200 \mu \mathrm{L}$ of the vaccine was administered at the base of the tail at the specified dose. For intranasal (IN) vaccination, mice were anaesthetised by intraperitoneal injection of $200 \mu \mathrm{L} 1 \mathrm{x}$ ketamine/xylazine solution in sterile PBS and $50 \mu \mathrm{L}$ of the vaccine was administered IN at the specified dose.

\subsubsection{Lymph node mapping}

Indian ink was diluted $1: 10$ in sterile PBS. $20 \mu \mathrm{L}$ was administered subcutaneously at the tail base between the dorsal and right lateral vein. After 2 hours, mice were euthanised, dissected and photographed to visualise ink drainage from each injection site. 


\subsubsection{Rodent diets}

Meat-free rat and mouse standard diet, zero-fibre, zero-starch rodent diet (SF15086), modified SF15-086 with $7.5 \%$ pectin, $7.5 \%$ inulin high fibre rodent diet (SF17-017), standard AIN93G rodent diet and modified AIN93G rodent diet with $8 \%$ Propolis formulated with cyclodextrin powder ( $25 \%$ and $75 \%$, respectively) (SF17-055) were purchased from Specialty Feeds, Western Australia. All foods were autoclaved or irradiated prior to feeding for sterility.

Unless otherwise stated, mice were maintained on meat-free rat and mouse standard diet. Other than the specified dietary alterations, paired diets in each experiment were designed to be otherwise nutritionally equivalent. Mice were administered foods ad libitum for the duration specified in each experiment.

\subsubsection{Sungold kiwifruit administration}

Zespri Sungold kiwifruit (Acintindia chinensis) was processed to a puree for intragastric (IG) administration. The surface of whole Sungold kiwifruit were sterilised by wiping with $70 \%$ ethanol and skin was removed. Kiwifruit was blended in Brabantia Stainless Steel Blender (Briscoes, Wellington, New Zealand) to a homogenous puree which was then put through a MACS $70 \mu \mathrm{M}$ cell strainer in order to reach a suitable consistency for administration. Kiwifruit puree was stored at $-20^{\circ} \mathrm{C}$ and defrosted at room temperature (RT) prior to administration. Control groups received fructose at $53 \mathrm{~g} / \mathrm{L}$, glucose at $58 \mathrm{~g} / \mathrm{L}$ and sucrose at $12 \mathrm{~g} / \mathrm{L}$ dissolved in autoclaved $\mathrm{H}_{2} \mathrm{O}$, matching the sugar profile of Sungold kiwifruit. Mice were administered solutions intragastrically using a stainless steel 22-gauge gavage needle. From day -14 to day -8 of experiments, $300 \mu \mathrm{L}$ of kiwifruit was administered, from day -7 to day -1 mice $400 \mu \mathrm{L}$ of kiwifruit was administered and from day 0 for the entirety of the experiment, $500 \mu \mathrm{L}$ of kiwifruit was administered. Lower doses of kiwifruit were 
administered over the first 2 weeks of the experiment as $500 \mu \mathrm{L}$ doses were not well tolerated by mice younger than 6 weeks of age.

\subsubsection{Bifidobacterium animalis subsp. lactis administration}

Bifidobacterium animalis subsp. lactis HN019 (B. animalis) was dissolved in autoclaved $\mathrm{H}_{2} \mathrm{O}$. Each mouse received $10^{9}$ colony forming units (CFU) of bacteria in $200 \mu \mathrm{L}$ IG using a stainless steel 22-gauge gavage needle. Control groups received the same volume of autoclaved $\mathrm{H}_{2} \mathrm{O}$.

\subsubsection{Short-chain fatty acid administration}

Solutions of single SCFAs were prepared by adding $200 \mathrm{mM}$ of sodium acetate, sodium butyrate or sodium propionate to autoclaved acidified drinking water, at a concentration of $200 \mathrm{mM}$ and administered to mice ad libitum for 21 days prior to vaccination and throughout the remainder of the experiment.

A mixed SCFA solution was prepared by adding sodium acetate $(70 \mathrm{mM})$, sodium butyrate $(20 \mathrm{mM})$ and sodium propionate $(30 \mathrm{mM})$, to autoclaved acidified water and was administered to mice ad libitum for 14 days prior to initial vaccination and throughout the remainder of the experiment.

\subsubsection{Euthanising mice}

Mice were euthanised by asphyxiation in $\mathrm{CO}_{2}$ chamber, followed by immediate cervical dislocation to ensure lethality. Mice also were euthanised by cervical dislocation or by cervical dislocation alone. Alternately, mice were euthanised by lethal anaesthesia by intraperitoneal injection of $1000 \mu \mathrm{L} 1 \mathrm{x}$ ketamine/xylazine solution in sterile PBS.

\subsubsection{Tissue collection and processing}

\subsubsection{Mouse serum collection}

For non-lethal serum collection at the lateral tail vein, mice were warmed with a heat lamp to promote vasodilation prior to blood collection. A disposable sterile 
surgical blade was used to make a small incision at the lateral tail vein and 3 to 4 drops of blood were collected into a Microvette gel serum collection tube. Pressure was applied to the incision until bleeding ceased. Alternatively, the submandibular vein was punctured and 3 to 4 drops of blood were collected into a Microvette gel serum collection tube.

For lethal serum collection, mice were euthanised by $\mathrm{CO}_{2}$ asphyxiation and blood was collected by cardiac puncture into a Microvette gel serum collection tube.

Following collection, blood samples were centrifuged for 5 minutes at 13,000 $\mathrm{g}$ and the serum fraction was stored at $-20^{\circ} \mathrm{C}$ for further analysis.

\subsubsection{Mouse faeces and caecal content collection}

For non-lethal faecal sample collection, mice were placed in clean, separate cages until faecal samples could be collected.

For lethal caecal content collection, mice were euthanised by $\mathrm{CO}_{2}$ asphyxiation and caecum was dissected and contents collected under sterile condition. All samples were stored in $0.6 \mathrm{~mL}$ microtubes at $-70^{\circ} \mathrm{C}$ for further processing.

\subsubsection{Bronchoalveolar lavage and nasal lavage collection}

To quantify secretory antibody in the pulmonary airways and nasal passage, bronchoalveolar lavage (BAL) and nasal lavage were collected. Mice were euthanised by lethal anesthesia as previously described and a longitudinal cut was made at the neck of the mouse, revealing the trachea. To collect BAL, a cannulated syringe was inserted into the trachea towards the lung and $500 \mu \mathrm{L}$ of sterile PBS was dispensed into the lung then the maximal amount was withdrawn into the syringe and collected, this was repeated once. To collect nasal lavage, a transverse, semi-lunar cut was made in the trachea, a cannulated syringe was inserted into the trachea towards the nose and $500 \mu \mathrm{L}$ of sterile PBS 
was dispensed rapidly through the nose and collected for analysis. The samples were centrifuged for 3 minutes at $13,500 \times g$ at $4^{\circ} \mathrm{C}$ and the supernatant was collected to exclude any red blood cells. Samples were stored at $-20^{\circ} \mathrm{C}$ for further analysis.

\subsubsection{Lung dissection}

To quantify viral load following influenza infection, lungs were collected. Mice were euthanised by cervical dislocation and a longitudinal cut was made through the upper torso of the mouse through the ribs. Lungs were excised, any surrounding connective tissue was removed, the tissue was then placed in a cryogenic vial and immediately snap-frozen in liquid nitrogen, before being stored at $-80^{\circ} \mathrm{C}$ for further analysis.

\subsubsection{Mouse faecal lysate}

To quantify secretory IgA in the faeces, faecal lysates were obtained. Briefly, fresh faecal pellets were weighed and homogenised in PBS containing $0.1 \%$ Tween 20 at $100 \mathrm{mg} / 100 \mu \mathrm{L}$. The samples were centrifuged for 10 minutes at $13,500 \times g$ at $4^{\circ} \mathrm{C}$ and the supernatant was collected. Samples were stored at $20^{\circ} \mathrm{C}$ until analysed.

\subsubsection{Lymph node cell isolation}

Following euthanasia by $\mathrm{CO}_{2}$ asphyxiation, LNs were collected into cold PBS and immediately stored on ice. For flow cytometry analysis of lymphocytes, LNs were mashed through a $70 \mu \mathrm{m}$ cell strainer and washed through with PBS to form a single-cell suspension. Cells were then centrifuged at $500 \times g$ for 5 minutes and the supernatants were discarded. Cells were resuspended in flow buffer for cell counting and preparation for flow cytometry.

For flow cytometry analysis of myeloid cells, LNs were digested to liberate cells from the organ. LNs were added to $1 \mathrm{~mL}$ of $1 \mu \mathrm{g} / \mathrm{mL}$ liberase TL and $100 \mu \mathrm{g} / \mathrm{mL}$ 
DNase I in IMDM, torn apart with 18 gauge needles and incubated at $37^{\circ} \mathrm{C}$ for 25 minutes. To LNs, $20 \mu \mathrm{L}$ of EDTA was added and incubated for a further 5 minutes at $37^{\circ} \mathrm{C}$. Cells were resuspended with a $1 \mathrm{~mL}$ pipette to liberate cells and then mashed through a $70 \mu \mathrm{m}$ cell strainer and washed through with IMDM. Cells were centrifuged at $500 \times g$ for 5 minutes and supernatants were discarded. Cells were resuspended in flow buffer for further cell counting and for use in flow cytometry.

\subsubsection{Production of inactivated viral vaccines}

\subsubsection{Formalin inactivation}

Formalin inactivation protocol was performed according to previous publication demonstrating complete viral inactivation ${ }^{213}$. Briefly, live HKx31 virus stock at $20,480 \mathrm{HAU} / \mathrm{mL}$ was incubated at $37^{\circ} \mathrm{C}$ in formalin at a final concentration of 0.02\% in PBS for 72 hours. Solutions were transferred to 10,000 MWCO Slide-ALyzer dialysis cassette and dialysed in a $1 \mathrm{~L}$ glass beaker containing $500 \mathrm{~mL}$ of DMEM. The process was repeated twice for 2 hours each and once overnight, on a IKA Colour Squid magnetic plate stirrer (Sigma). Dialysed vaccine was diluted to $1000 \mathrm{HAU} / \mathrm{mL}$ and stored at $-70^{\circ} \mathrm{C}$ until required.

\subsubsection{Ultraviolet light inactivation}

Ultraviolet (UV) irradiation inactivation protocol was performed according to Zou and colleagues, demonstrating complete viral inactivation following greater than 30 minutes of UV irradiation ${ }^{214}$. Briefly, live influenza A HKx31 virus stock at 20,480 HAU/mL was spread in a thin layer at $1.5 \mathrm{~mL}$ per sterile petri dish with

a cell scraper. Petri dishes were placed in a Herasafe Class II Biological Safety Cabinet (Thermofisher Scientific) and UV radiation was applied for 45 minutes, ensuring no shadows blocked exposure of the viral suspension to radiation. Inactivated vaccine was resuspended at 10,000 HAU/ mL in PBS and stored at - 
$70^{\circ} \mathrm{C}$ until required. Annual testing ensures UV radiation in the safety cabinet reaches the required UV radiation safety standards for sterilisation.

\subsubsection{Enzyme-Linked Immunosorbent Assay (ELISA)}

\subsubsection{TIV-specific IgG and IgG1}

Levels of TIV-specific IgG and IgG1 antibody in mouse serum were determined by ELISA. Briefly, each well of a 96 well Nunc Maxisorb plates was coated with $100 \mu \mathrm{L}$ of Influvac ${ }^{\circledR}$ diluted to $25 \mu \mathrm{L} / \mathrm{mL}$ in PBS and incubated overnight at $4^{\circ} \mathrm{C}$. Non-specific protein binding was blocked with the addition of $150 \mu \mathrm{L}$ of $10 \%$ FBS in PBS and incubated for 2 hours at RT. Serum samples were serially diluted in $10 \%$ FBS in PBS and $100 \mu \mathrm{L}$ was added to each well. The plates were then incubated for 2 hours at RT. HRP-conjugated anti-mouse IgG and IgG1 secondary antibody were diluted in 10\% FBS in PBS at 1:5000 or 1:1000. $100 \mu \mathrm{L}$ was added to each well and plates were incubated for 2 hours at RT. Finally, 100 $\mu \mathrm{L}$ of OptEIA TMB substrate was added and after 5-10 minutes the reaction was stopped with $50 \mu \mathrm{L}$ of $1 \mathrm{M}$ sulphuric acid. The absorbance was read on Versa Max microplate reader at $450 \mathrm{~nm}$. Before the initiation of each step, plates were washed five times with $0.05 \%$ Tween 20 in PBS, except following the blocking step. Relative antibody levels were expressed as optical density at $450 \mathrm{~nm}$ (O.D. $450)$ at the appropriate non-saturating serum concentration.

\subsubsection{HKx31 virus-specific $\lg G$, $\lg G 1$ and $\lg A$}

Levels of HKx31 virus-specific IgG and IgG1 antibody in mouse serum and IgA in mouse BAL and nasal lavage were determined by ELISA assay. Briefly, 96 well Nunc Maxisorb plates were coated with $100 \mu \mathrm{L}$ of HKx31 virus stock diluted to $10 \mathrm{HAU} / \mu \mathrm{L}$ in PBS and incubated overnight at $4^{\circ} \mathrm{C}$. Non-specific protein binding was blocked by addition of $150 \mu \mathrm{L}$ of $10 \%$ FBS in PBS and plates were incubated for 2 hours at RT. Samples were serially diluted in 10\% FBS in PBS and $100 \mu \mathrm{L}$ was added per well. Plates were incubated for 2 hours at RT. 
HRP-conjugated anti-mouse IgG, IgG1 and IgA secondary antibodies, were diluted in 10\% FBS in PBS at 1:5000, 1:1000 and 1:4000, respectively. Each well received $100 \mu \mathrm{L}$ of diluted secondary antibody and incubated for 2 hours at RT. Finally, $100 \mu \mathrm{L}$ of OptEIA TMB substrate was added to each well and 5-10 minutes later the reaction was stopped with the addition of $50 \mu \mathrm{L} 1 \mathrm{M}$ sulphuric acid to each well. The absorbance was read on Versa Max microplate reader at $450 \mathrm{~nm}$. Before each step, plates were washed five times with $0.05 \%$ Tween 20 in PBS.

\subsubsection{3 $\lg \mathrm{A}$}

IgA levels in the serum and faeces were measured using mouse IgA Ready-SETGo ELISA kit according to the manufacturer's instructions. Samples were serially diluted as needed for accurate readings.

\subsubsection{Quantitative PCR}

\subsubsection{Lung RNA extraction}

The right lung was placed in a sterile petri dish, roughly chopped with a disposable steel blade and placed in a cliplock tube with $1 \mathrm{~mL}$ of TRIzol reagent and two stainless steel beads. Samples were homogenised in a tissue lyser (Tissue Lyser II, Qiagen) for 2 minutes at $30 \mathrm{~Hz}$. This was repeated with samples switched to the opposite arm of the tissue lyser to ensure equal homogenisation between samples. Samples were centrifuged for $10,000 \times g$ for 3 minutes at $4^{\circ} \mathrm{C}$ and the supernatants were transferred to a sterile $2 \mathrm{~mL}$ microtube. Each sample received $200 \mu \mathrm{L}$ of chloroform, shaken by hand for 15 seconds and left to rest for 5 minutes at RT. Samples were then centrifuged at $12,000 \times g$ for 15 minutes at $4^{\circ} \mathrm{C}$. The transparent, colourless top layer was transferred from each sample to a sterile $1.7 \mathrm{~mL}$ microtube and $500 \mu \mathrm{L}$ of isopropanol was added. Samples were incubated at RT for 10 minutes then centrifuged for $12,000 \times g$ for 10 minutes at $4^{\circ} \mathrm{C}$. The supernatants were discarded and each sample was washed with $1 \mathrm{~mL}$ 
of $70 \%$ ethanol and centrifuged at $10,000 \times g$ for 5 minutes at $4^{\circ} \mathrm{C}$. The supernatants were discarded and samples were centrifuged at $10,000 \times g$ for 1 minute at $4^{\circ} \mathrm{C}$. Excess ethanol was carefully removed, leaving the RNA pellet in the microtube. The pellet was left to air dry for 5-10 minutes and resuspended in $40 \mu \mathrm{L}$ of DPEC-treated $\mathrm{H}_{2} \mathrm{O}$. RNA concentration and purity was measured on a ND-1000 Nanodrop spectrophotometer (Thermofisher Scientific). Samples were stored at $-70^{\circ} \mathrm{C}$ until required.

\subsubsection{RNA to cDNA conversion}

RNA was converted to complementary DNA (cDNA) using the High Capacity RNA to cDNA Kit according to the manufacturers specifications. The reaction was run in a Veriti 96-well Thermo Cycler (Thermofisher Scientific), incubated for 60 minutes at $37^{\circ} \mathrm{C}$ followed by 5 minutes at $95^{\circ} \mathrm{C}$ to stop the reaction and then held at $4^{\circ} \mathrm{C}$. cDNA was stored at $4^{\circ} \mathrm{C}$ for up to 3 days or at $-20^{\circ} \mathrm{C}$ for long term storage until required.

\subsubsection{Faecal bacteria DNA extraction}

Bacterial DNA was extracted from faecal samples using QIAamp Fast DNA Stool Mini Kit according to the manufacturer's instructions. DNA concentration and purity was measured on a ND-1000 Nanodrop spectrophotometer (Thermofisher Scientific). Samples were stored at $-20^{\circ} \mathrm{C}$ until required.

\subsubsection{Quantitative PCR}

Determination of influenza virus viral load in lung

A custom FAM-labelled TaqMan influenza A probe was purchased from Life Technologies for RNA analysis as described previously ${ }^{48}$. This consisted of influenza A virus sense primer (5' -AAGACCAATCCTGTCACCTCTGA-3' ), antisense primer ( $5^{\prime}$-CAAAGCGTCTACGCTGCAGTCC-3' $)$ and probe (FAM-5' -TTTGTGTTCACGCTCACCGT-3' -TAMRA). All primers were 
specific for the influenza A virus matrix protein. VIC-labelled TaqMan Gapdh probes were used as an endogenous control (Assay ID NM_008084.2; Thermofisher Scientific). A qPCR reaction mix was first prepared by combining per reaction; $10 \mu \mathrm{L}$ TaqMan master mix, $1 \mu \mathrm{L}$ Gapdh housekeeping gene probe, 1 $\mu \mathrm{L}$ influenza A virus probe and $5 \mu \mathrm{L}$ of DPEC-treated $\mathrm{H}_{2} \mathrm{O}$. To each well of an optical 96-well reaction plate with barcode, $17 \mu \mathrm{L}$ of prepared qPCR reaction mix and $3 \mu \mathrm{L}$ of the cDNA sample were added. The plate was covered with an optical adhesive film and centrifuged for approximately 30 seconds at $500 \times \mathrm{g}$. All samples were run in duplicate and each plate included an $\mathrm{H}_{2} \mathrm{O}$ sample as a negative control. qPCR was run on a QuantStudio 7 Flex PCR system (Thermofisher Scientific) using the standard instrument settings for SYBR green 96-well plate assay.

\section{Determination of predominant bacteria in faeces}

Proportions of dominant bacteria in the faeces were determined by quantitative PCR as previously described ${ }^{215}$. A qPCR reaction mix was prepared by combining per reaction; $10 \mu \mathrm{L}$ SYBR green PCR master mix, $1 \mu \mathrm{L}$ each of forward and reverse primers (Supplementary table 1) and $6 \mu \mathrm{L}$ of DPEC-treated $\mathrm{H}_{2} \mathrm{O}$. To each well of an optical 96-well reaction plate with barcode, $18 \mu \mathrm{L}$ of prepared qPCR reaction mix and $2 \mu \mathrm{L}$ of the DNA sample (standardised to $1 \mathrm{ng} / \mu \mathrm{L}$ ) were added. The plate was covered with an optical adhesive film and centrifuged for approximately 30 seconds at $500 \times g$. All samples were run in duplicate and each plate included an $\mathrm{H}_{2} \mathrm{O}$ sample as a negative control. qPCR was run on a QuantStudio 7 Flex PCR system (Thermofisher Scientific) using the standard instrument settings for fast TaqMan 96-well plate assay. 


\subsubsection{Total protein quantification}

Total protein was quantified using the Pierce bicinchoninic acid (BCA) protein assay according to the manufacturers instruction. Samples were serially diluted in PBS in order to obtain optimal sample protein concentrations for accurate measurements.

\subsubsection{TIV vaccine AF488 protein labelling}

Initially, TIV was concentrated to $2 \mathrm{mg}$ protein/mL by placing on a Vivaspin 20 10,000 MWCO column and centrifuged at $3000 \times g$. Protein concentration was determined by BCA assay and vaccine was diluted to $2 \mathrm{mg} / \mathrm{mL}$ in PBS for labelling. Concentrated TIV vaccine was labelled with Alexa Fluor 488 dye using the Alexa Fluor 488 Protein Labelling Kit (Thermofisher Scientific, Auckland, New Zealand) according to the manufacturer's instructions. When specified in the experiment, the vaccine was subsequently washed. To do so, the labelled vaccine was placed on a Vivaspin 20 10,000 MWCO column and topped up to $20 \mathrm{~mL}$ with sterile PBS, this was repeated once. Following labelling, protein concentrations were measured by BCA assay and diluted in sterile PBS to equal concentration as unlabeled TIV vaccine. All labelling procedures were performed in sterile conditions.

\subsubsection{Flow cytometry}

\subsubsection{Controls}

Controls used for flow cytometry experiments included; unstained cells, viability dye single-stained cells, fluorescence minus one (FMO) stained cells and single-stained compensation beads. 


\subsubsection{Cell surface staining}

Flow cytometry antibodies were titrated for optimal concentration prior to use in experiments. Antibodies were centrifuged at $12,000 \times g$ and only the supernatant was taken to exclude the use of aggregated antibody. Single cell suspensions from LNs were obtained as previously described (in section 1.1.1) and counted using trypan blue exclusion. Between 1-5 × $10^{6}$ cells were plated per well of a 96-well V-bottom plate, centrifuged at $500 \times g$ for 3 minutes and supernatants were discarded. If fixable viability dye was used, samples were resuspended in $50 \mu \mathrm{L}$ of viability dye diluted in PBS and incubated for 10 minutes on ice. Samples were washed by addition of $150 \mu \mathrm{L}$ of flow buffer and centrifuged for 3 minutes at $500 \times g$, supernatants were discarded. All subsequent washes were performed in this manner. Samples were resuspended in $50 \mu \mathrm{L}$ of flow buffer containing anti-CD16/CD32 antibody for 10 minutes to prevent non-specific binding of antibodies to Fc receptor-expressing cells. If biotinylated antibodies were used, cells were resuspended in $50 \mu \mathrm{L}$ of flow buffer containing the biotinylated antibody, incubated for 30 minutes at $37^{\circ} \mathrm{C}$ then washed twice. Cells were then resuspended in $50 \mu \mathrm{L}$ of cell surface antibodies diluted in flow buffer, including any streptavidin-conjugated fluorophores if used, and were incubated for 30 minutes at $4^{\circ} \mathrm{C}$, washed twice and resuspended in $150 \mu \mathrm{L}$ of flow buffer for acquisition. DAPI viability dye was diluted in flow buffer and added to samples immediately prior to acquisition.

\subsubsection{Intracellular staining}

For antigens located within the cell, cells were permeabilised prior to staining to allow antibody to enter the cell. Cells were fixed and permeabilised using the FoxP3/Transcription Factor Staining Buffer Set (eBioscience) as per manufacturer's specifications. Briefly, cells were stained for cell surface antigens as described previously (In X.X.X) and washed twice. Prior to intracellular staining, one part fixation/permeabilisation concentrate was diluted with three 
parts fixation/permeabilisation diluent to yield a $1 \mathrm{x}$ fixation/permeabilisation solution and one part permeabilisation buffer was diluted with nine parts PBS to yield $1 x$ permeabilisation buffer. Cells were resuspended in $100 \mu \mathrm{L}$ of fixation/permeabilisation solution and incubated at $4{ }^{\circ} \mathrm{C}$ for 30 minutes. Cells were washed twice with $150 \mu \mathrm{L}$ of permeabilisation buffer, centrifuged at $500 \mathrm{x}$ $g$ for 3 minutes and supernatants were discarded. Cells were then resuspended in $50 \mu \mathrm{L}$ of intracellular antibodies diluted in permeabilisation buffer and incubated for 1 hour at RT. Cells were washed twice and resuspended in $200 \mu \mathrm{L}$ of flow buffer for acquisition.

\subsubsection{Acquisition and analysis}

Samples were analysed on either an LSR-II special order product (BD) or an LSR Fortessa (BD) flow cytometer.

The LSR-II was equipped with the following lasers and detectors: Ultra violet laser (355 nm) UV740/35, UV450/50 and UV379/28. Violet laser (405 nm) V780/60, V720/40, V660/20, V605/40, V560/40, V525/50 and V450/50. Blue laser (488 nm) B705/70, B515/20 and B488/10. Green laser (532 nm) G780/60, G610/20 and G575/26. Red laser (640 nm) R780/60, R710/50 and R670/14.

The LSR Fortessa was equipped with the following lasers and detectors: Violet laser (405 nm) V780/60, V710/20, V660/20, V610/20, V560/40, V525/50 and V450/50. Blue violet laser (445 nm) BV504/12. Blue laser (488 nm) B685/35, B515/20 and B488/10. Green laser (532 nm) G780/60, G695/40, G670/30, G610/20 and G575/26. Red laser (640 nm) R780/60, R710/50 and R670/14.

All fluorophores were detected using laser and detector combinations that best matched their excitation and emission profile.

Following acquisition, FCS files were analysed using FlowJo software (Tree Star, San Carlos, CA, USA). For all flow cytometry analysis, cells were gated first by 
excluding doublets using forward and side scatter. Dead cells were removed from analysis by gating on viability dye negative cells. Cells of interest were identified by forward and side scatter profile before immunophenotyping (Figure 2.1).

\subsubsection{Statistics}

Statistical analysis was performed using Prism software (GraphPad Prism Version 6.0 for MacIntosh, GraphPad Software Incorporated, USA). Figure legends state the statistical analysis used for each experiment.

For experiments comparing only two groups, statistics were obtained using a Mann Whitney test. In experiments with three or more groups, statistics were obtained using a one-way ANOVA with Bonferroni's post-test. Tukey's post-test was applied when comparing every mean. Dunnett's post-test was applied when comparing every mean with a control mean. Bonferroni's post-test was applied when a set of means were selected to compare.

In experiments comparing groups between time points, statistics were obtained using a two-way ANOVA with a Bonferroni's post-test. Relevant statistics are shown in figures. 
A)

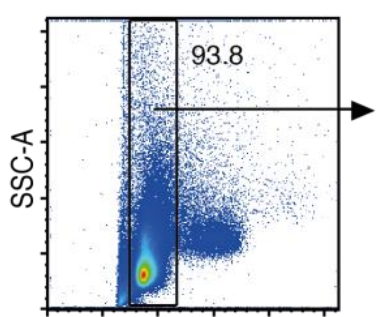

FSC-W

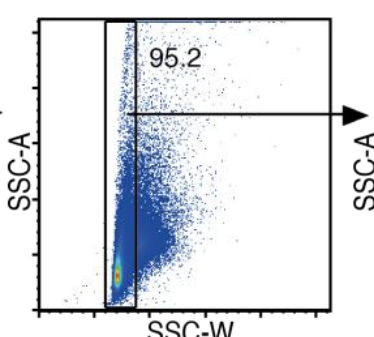

SSC-W

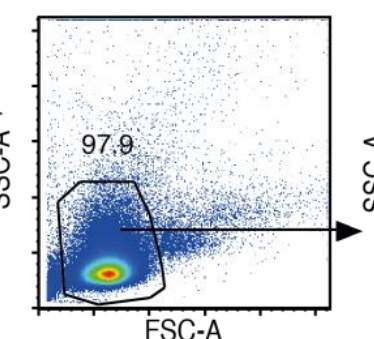

FSC-A

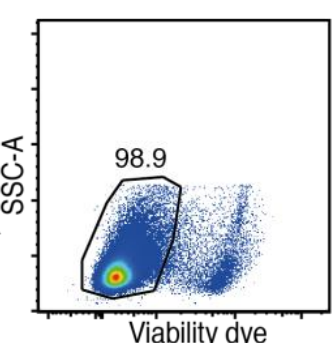

Viability dye

B)

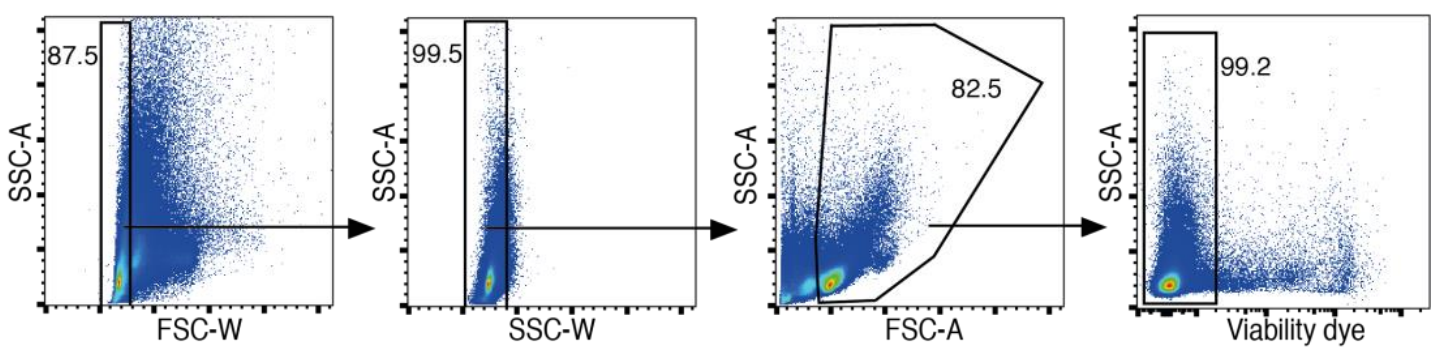

Figure 2.1: Pre-gating strategy for flow cytometry analysis. Representative pre-gating strategy to select for single, viable cells of interest when analysing (A) lymphocytes or (B) myeloid cells and lymphocytes. 
3 Evaluating the gut microbiota influence on antibody

responses to influenza vaccination 


\subsection{Introduction}

TIV vaccination acts by inducing the production of IgG isotype antibodies which protect against infection and a critical threshold of anti-influenza antibodies need to be mounted to confer protection, termed seroconversion ${ }^{107,108}$. However, there is a high degree of variability in the titre of antibody reached across individuals within a population, with some individuals not reaching seroconversion. The mechanisms by which the vaccine elicits high levels of protective antibody in some individuals, whilst inducing weak responses in others, remains incompletely understood. Interestingly, transcriptome analysis of human PBMCs following TIV vaccination has revealed that expression of TLR-5, the PRR for bacterial flagellin ${ }^{35}$, was strongly correlated with the level of virus-neutralising antibody in the serum of individuals ${ }^{36}$. Further investigations indicated that antibody responses to TIV were severely impaired in TLR-5 deficient mice, as well as GF and antibiotic-treated mice, illustrating that microbial flagellin may act as an adjuvant to boost host antibody responses to the vaccine ${ }^{158}$. TLR-5 signalling on $\mathrm{M} \varphi$ was demonstrated to be important for expression of CD86 and IL-6, and direct signalling through TLR-5 on B cells was shown to promote differentiation to PCs, both crucial for robust TIV-specific antibody production ${ }^{158}$.

There is a growing body of research demonstrating that host recognition of immunostimulatory components of gut microbes or their metabolites can influence the development and function of many immune cell subsets to enhance local and systemic immune responses. Gut microbial signals influence the development of multiple innate immune cell populations systemically by altering haematopoesis in primary lymphoid organs, via both TLR signalling ${ }^{37}$ and microbiota-produced circulating SCFAs ${ }^{38}$. Additionally, the microbiota is 
required for maintaining the ability for innate immune cell populations to mount effective responses to infectious challenges. Type I interferon (IFN-I) production following viral encounter by mononuclear phagocytes is impaired in GF and antibiotic-treated mice, leading to a failure to prime NK cells and a decrease in viral control ${ }^{39}$. Microbiota signalling has also been shown to be instrumental for inflammasome activation in DCs following influenza infection, promoting the migration of DCs to secondary lymphoid organs and expression of costimulatory molecules, that in turn promote $\mathrm{T}$ and $\mathrm{B}$ cell responses ${ }^{40}$. Antibiotic administration also leads to decreased numbers of $M \varphi$ in the lung following influenza infection, which express lower levels of CD86 ${ }^{41}$. Microbiota-derived SCFAs directly enhance peripheral PC differentiation to boost baseline concentrations of IgG antibody in the blood ${ }^{41}$. This demonstrates the ubiquitous and systemic influence of the gut microbiota on various cell subsets of the innate and adaptive immune system. As the humoral immune response requires the concerted action of a number of immune cell populations, including $\mathrm{B}$ cells, $\mathrm{T}$ cells, DCs and $M \varphi$, it is likely that previously demonstrated microbiota effects are playing a role in the humoral immune response to TIV vaccination.

Antibiotic treatment reduces intestinal bacterial load that in turn lowers host systemic exposure of the host to microbial stimuli, including circulating microbial metabolites such as SCFAs ${ }^{42}$ and flavonoid degradation products ${ }^{43}$, as well as bacterial cell components in the serum, such as peptidoglycan ${ }^{44}$. Administration of broad spectrum antibiotics to adult mice does not completely ablate the microbiota ${ }^{45}$ or impair early immune system development as is observed in GF mice ${ }^{7}$ and so serves as a physiologically relevant model of the dysbiotic phenotype in the human population ${ }^{13}$ to examine microbiota influence on immune responses to TIV. 


\subsection{Aims}

Based on the ubiquitous influence of the microbiota on multiple immune cell compartments, it is highly likely the host is integrating many signals from the microbiota in addition to the requirement for flagellin signalling via TLR-5 to mount optimal antibody responses to TIV vaccination. Therefore, in this chapter the impact of alterations to microbial signalling on a number of cellular processes in the generation of the humoral immune response to TIV vaccination were examined by manipulating microbiota populations through antibiotic treatment.

Specific aims:

1) To develop a model of oral antibiotic administration that reduces antibody response to TIV vaccination.

2) To assess changes in immune cell frequency and function in the humoral immune response to TIV following microbiota alteration with antibiotic treatment. 


\subsection{Results}

\subsubsection{Intact microbiota communities are required for antibody response to influenza vaccination}

We first sought to establish if altering the gut microbiota by administration of oral antibiotics would lead to reduced vaccine-specific antibody production. In a preliminary experiment conducted by Dr. Hazel Poyntz (MIMR), mice were administered a broad-spectrum antibiotic cocktail, consisting of vancomycin, neomycin, ampicillin and metronidazole $(\mathrm{ABX})$, in the drinking water for 14 days prior to TIV vaccination and throughout the experiment. Vaccine-specific total IgG antibody and the IgG1 isotype were quantified at 14 and 28 days post-vaccination (Figure 3.1A). IgG1 antibodies were chosen to be measured because they are the dominant isotype produced in response to TIV vaccination in humans ${ }^{13}$ and mice mb,47. $^{4}$.

ABX treatment led to a striking reduction in total IgG (Figure 3.1B) and IgG1 isotype (Figure 3.1C) in the serum at both 14 and 28 days after vaccination. However, it was observed that mice are prone to becoming dehydrated during this long period of $A B X$ treatment. Based on this, the duration of $A B X$ administration was reduced to just three days prior until seven days post-vaccination (Figure 3.1D), minimising dehydration as a confounding factor. Indeed, only ten days $A B X$ treatment was sufficient to significantly reduce the serum concentration of vaccine-specific total IgG (Figure 3.1E) and IgG1 (Figure $3.1 F)$ antibody at 14 and 28 days following vaccination.

To confirm that this ten-day course of oral ABX was indeed altering gut microbial communities, faeces were analysed by qPCR prior to ABX (day -3), on the day of vaccination (day 0) and following ten-days of ABX treatment (day 7). 
Phyla-specific 16S rRNA gene-targeted primers for Firmicutes, Actinobacteria, Deferribacteres, Candidatus Saccharibacteria, Verrucomicrobia, Tenericutes and $\alpha, \beta$ and $\gamma$ proteobacteria were used in the assay and phyla representation was expressed as a proportion of total bacterial RNA quantified against a universal $16 \mathrm{~S}$ rRNA sequence. Actinobacteria, Deferribacteres, Candidatus Saccharibacteria, Tenericutes and $\alpha$ Proteobacteria phyla were undetectable in the faeces by this assay (Figure 3.1G). Following only three days of ABX, the frequencies of Firmicutes and Verrucomicrobia were diminished, while Actinobacteria and Bacteroidetes increased in frequency (Figure 3.1G). After ten days of $\mathrm{ABX}$ the faecal microbiota was further altered, with an expansion of Verrucomicrobia, Firmicutes and $\beta$ proteobacteria (Figure 3.1G). It should be noted that this experiment was repeated, however the DNA extracted from the faeces was of poor quality, with low A260/A230 ratios by spectrophotometric analysis, and could not be used for qPCR (Supplementary figure 1A and B).

These data demonstrate that ten days $A B X$ treatment significantly altered microbial phyla composition and this short treatment was able to recapitulate the detrimental effect on antibody production without the confounding factor of dehydration. Therefore, in this model, signals from the gut microbiota are important in antibody production to TIV vaccination. 
(A)
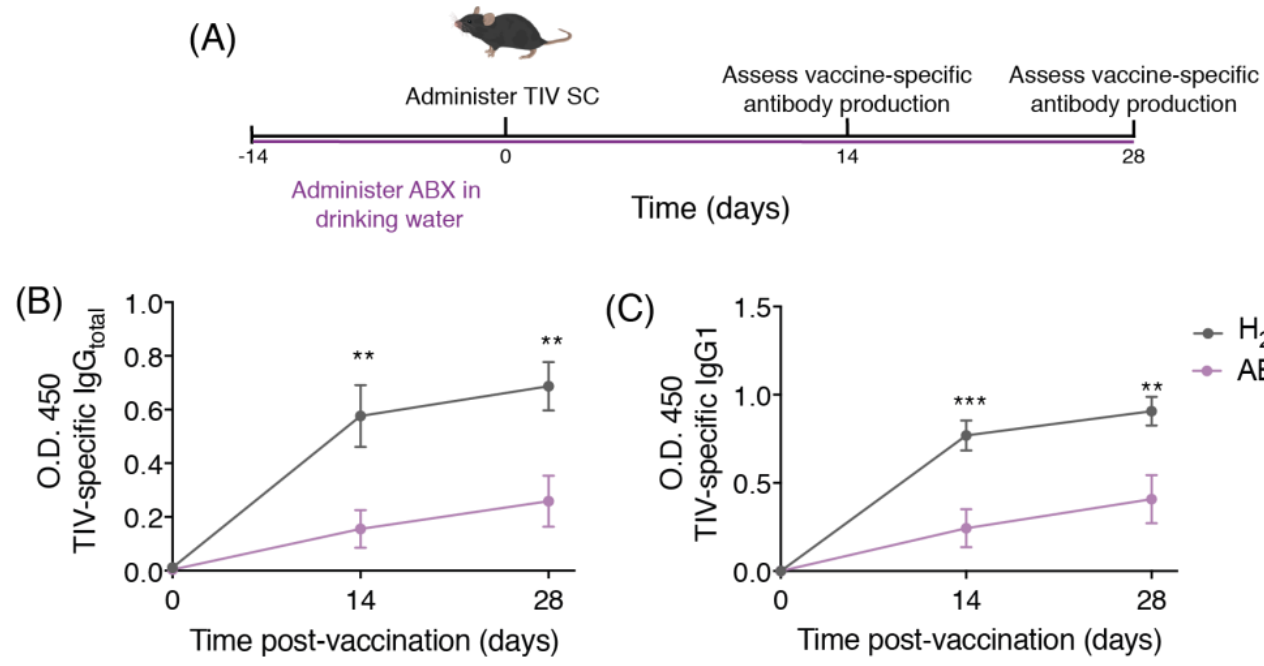

(C)

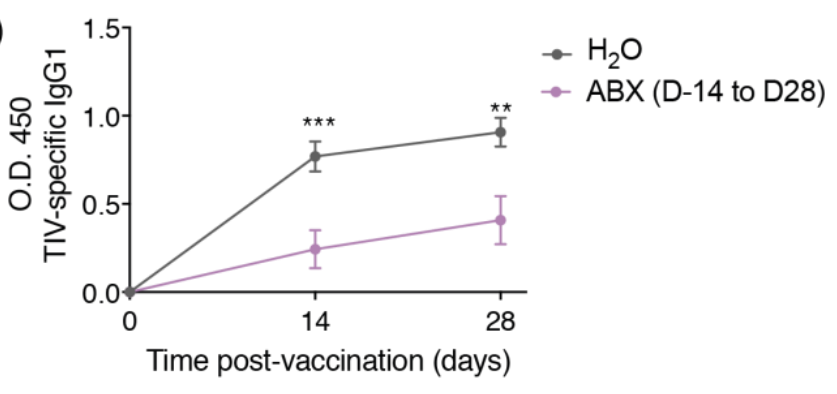

(D)
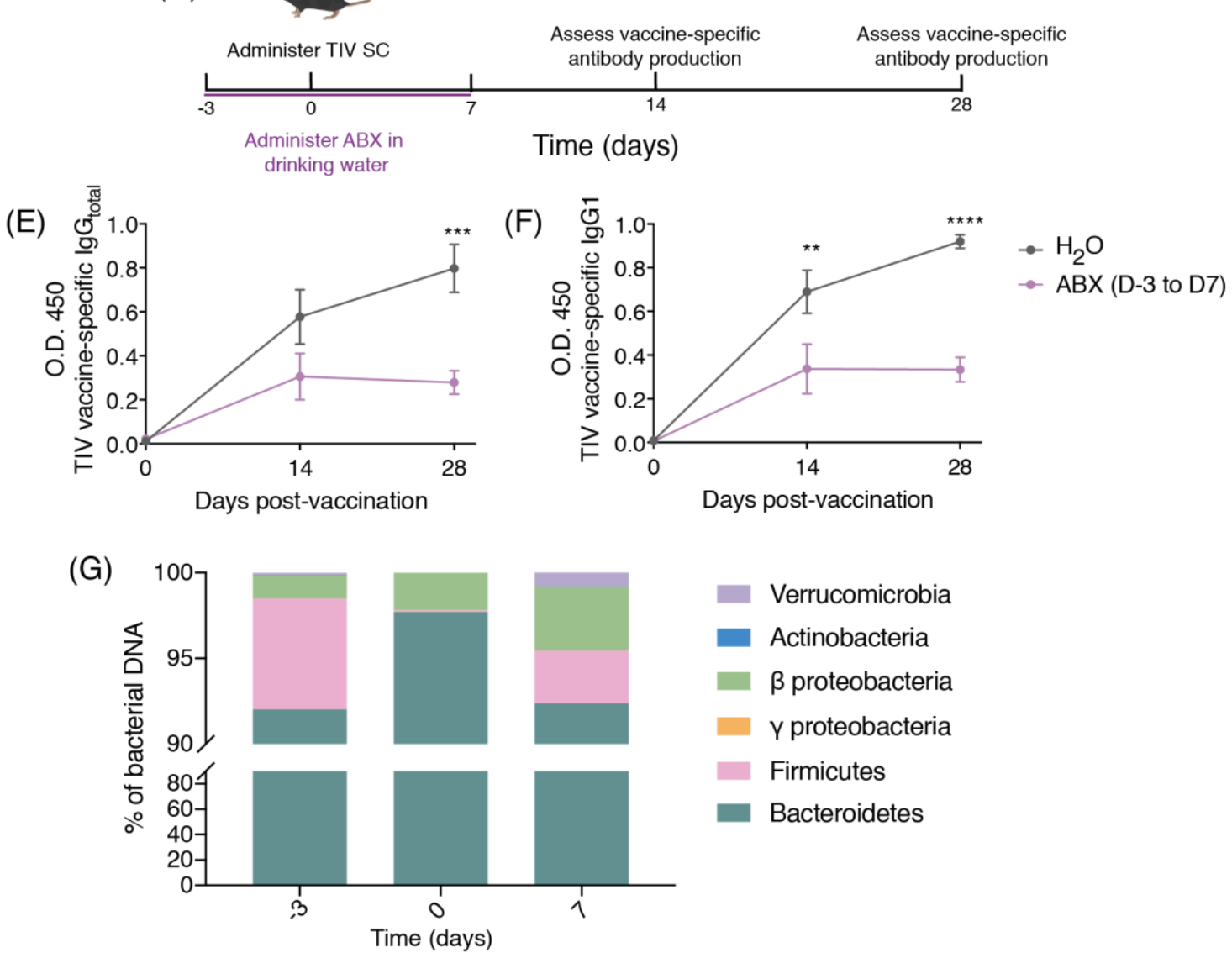
Figure 3.1: Administration of antibiotics is detrimental to vaccine-specific antibody production and significantly alters faecal microbial communities. Experiment in (A-C) was conducted by Dr. Hazel Poyntz. (A) Experimental outline for long-term administration of oral antibiotics and TIV vaccination. C57BL/6 mice received a broad-spectrum antibiotic cocktail $(\mathrm{ABX})$ in the drinking water, consisting of vancomycin $(0.5 \mathrm{mg} / \mathrm{mL})$, neomycin $(1 \mathrm{mg} / \mathrm{mL})$, ampicillin $(1 \mathrm{mg} / \mathrm{mL})$ and metronidazole $(1 \mathrm{mg} / \mathrm{mL})$, or drinking water alone $\left(\mathrm{H}_{2} \mathrm{O}\right)$ ad libitum for 14 days prior to initial vaccination and throughout the entirety of the experiment. Mice were vaccinated with $200 \mu \mathrm{L} 25 \%$ TIV in PBS. At 14 and 28 days post-vaccination, serum was collected for measurement of TIV-specific antibody by ELISA. TIV-specific (B) total IgG and (C) IgG1 at days 14 and 28 following vaccination. (D) Experimental outline for short-term administration of oral antibiotics and TIV vaccination. C57BL/6 mice received $\mathrm{ABX}$ or $\mathrm{H}_{2} \mathrm{O}$ ad libitum, for three days prior to initial vaccination until seven days following vaccination. Mice were vaccinated with $200 \mu \mathrm{L} 25 \%$ TIV in PBS. At 14 and 28 days following vaccination, serum was collected for measurement of TIV-specific antibody by ELISA. TIV-specific (E) total IgG and (F) IgG1 at days 14 and 28 post-vaccination. (E-F) is representative of 3 independent experiments. (G) Faecalresident bacterial frequencies within a cage of mice throughout $A B X$ treatment was determined by qPCR using 165 rRNA gene-targeted group-specific primers for Bacteroidetes, Firmicutes, Proteobacteria, Actinobacteria, Verrucomicrobia, Candidatus Saccharibacteria, Deferribacteres and Tenericutes, and proportions were quantified against a universal $16 S$ sequence. Proportions of bacteria are presented as the group mean. Data shown is $n=4-5$ mice per group. Statistical analysis of antibody titres was performed using Two-Way ANOVA with Sidak's post-test showing mean \pm SEM. ${ }^{* *} \mathrm{p} \leq 0.01,{ }^{* * *} \mathrm{p} \leq 0.001,{ }^{* * *} \mathrm{p} \leq 0.0001$. 


\subsubsection{Subcutaneous TIV vaccination induces potent B cell responses in inguinal lymph nodes}

Having established a model in which antibiotics reduce antibody responses to TIV vaccination, immune cell processes in the humoral immune response to TIV were next investigated in order to determine the cellular basis for the reduction in antibody production to vaccination following antibiotic treatment. To assess this, the impact of $\mathrm{ABX}$ on the GC response to vaccination first had to be assessed. However, in order to analyse this response, the tail base dLNs needed to be determined.

To assess vaccine drainage, mice were injected with $10 \%$ indian ink in PBS or PBS alone, between the dorsal and right or left lateral veins, and were sacrificed two hours later. As expected, dLNs could not be visualised with PBS injection (Figure 3.2A). Following injection on the right or left of the tail base ink could consistently be visualised at both the axillary and inguinal LNs on both sides (Figures 4.2B and $\mathrm{C}$ and quantified in Figure 3.2D). Ink drainage could not be visualised at any other LNs. To validate that SC vaccination in the tail was indeed draining to inguinal and axillary LNs, flow cytometry analysis of inguinal or axillary LN B cell and PC populations was performed at 14 days following influenza TIV vaccination and compared to LNs of mice administered PBS (Gating strategy figure 3.3). While the frequency of B cells was not increased in either set of LNs following vaccination, there was a significant increase in B cell number in the inguinal LNs following vaccination, reflecting the increased cellularity (Figure 3.2E). IgG1 producing cells could also be tracked using flow cytometry providing a cellular correlate to IgG1 antibody titres. This allows for antibody titres to be correlated with IgG1 producing cells detected by flow cytometry, as fluorescent antibodies specific for total IgG are not available. There was a significantly increased frequency and number of GC B cells (Figure 3.2F), 
IgG1 ${ }^{+}$GC B cells (Figure 3.2G) and IgG1 ${ }^{+}$PCs (Figure 3.2H) in the inguinal LNs following TIV vaccination. However, in axillary LNs the number and frequency of these populations were comparable to PBS administered mice.

This data shows that the inguinal LNs are the primary dLN of SC TIV vaccination in the tail base and subsequent analyses of cellular responses were performed with these LNs. 
(A)

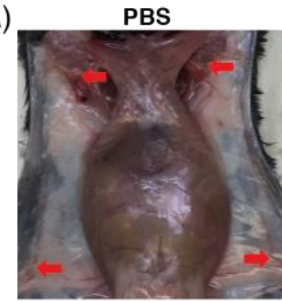

(B)

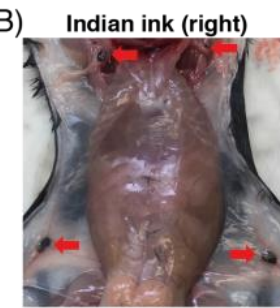

(C)

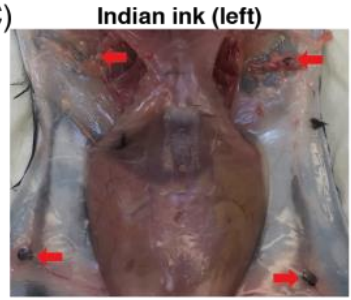

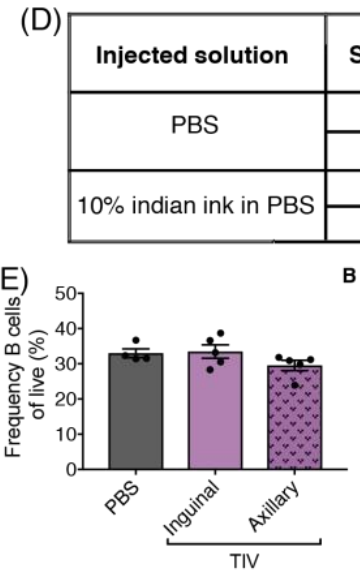

(G)

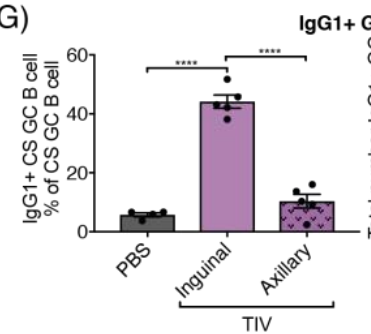

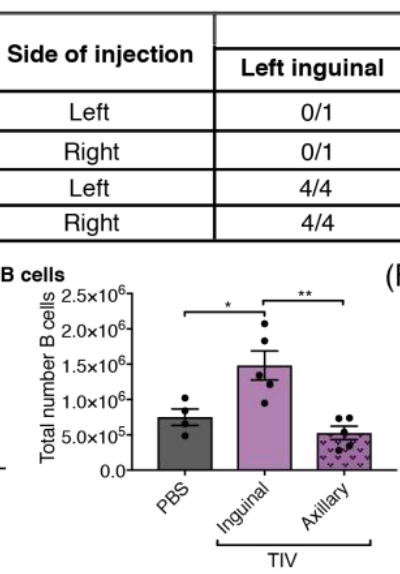

$(F)$

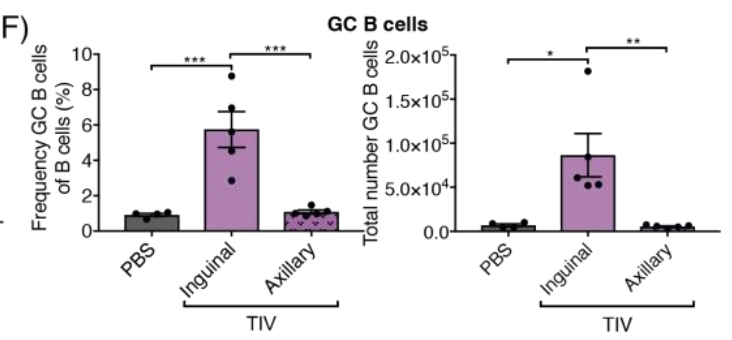

( $\mathrm{H})$

Draining lymph node

\begin{tabular}{|l|l|l|} 
Right inguinal & Left axillary & Right axillary \\
\hline
\end{tabular}

\begin{tabular}{|c|c|c|}
\hline $0 / 1$ & $0 / 1$ & $0 / 1$ \\
\hline $0 / 1$ & $0 / 1$ & $0 / 1$ \\
\hline $4 / 4$ & $4 / 4$ & $4 / 4$ \\
\hline $4 / 4$ & $4 / 4$ & $4 / 4$ \\
\hline
\end{tabular}


Figure 3.2: SC TIV vaccination at the tail base drains to inguinal $L N$ and induces potent $B$ cell responses at 14 days post vaccination. (A-C) Representative images showing $\mathrm{LN}$ drainage two hours following $20 \mu \mathrm{L}$ SC injection of (A) PBS between the dorsal and right lateral vein, (B) $10 \%$ indian ink in PBS between the dorsal and right lateral veins and (C) 10\% indian ink in PBS between the dorsal and left lateral veins of the tail of C57BL/6 mice. Red arrows show LN. This experiment is summarised in (D), indicating the number of mice in which drainage was observed to each LN. 14 days post-SC vaccination with $200 \mu \mathrm{L} 25 \%$ TIV in PBS or PBS alone at the tail base, inguinal or axillary LNs were analysed by flow cytometry. For PBS group analysis, cells from one inguinal and one axillary LN were pooled. Frequency and total number of (E) B cells, (F) GC $B$ cells, $(G) \mathrm{IgG1}^{+} \mathrm{GC}$ B cells and $(\mathrm{H}) \mathrm{IgG1}{ }^{+} \mathrm{PC}$. Data shown is $\mathrm{n}=4-5$ per group. Statistical analysis of (E-H) was performed using One-Way ANOVA with Tukey's post-test showing mean \pm SEM. ${ }^{*} \mathrm{p} \leq 0.05,{ }^{* *} \mathrm{p} \leq 0.01,{ }^{* * *} \mathrm{p} \leq 0.001,{ }^{* * * *} \mathrm{p} \leq 0.0001$. 


\section{A) B cells}
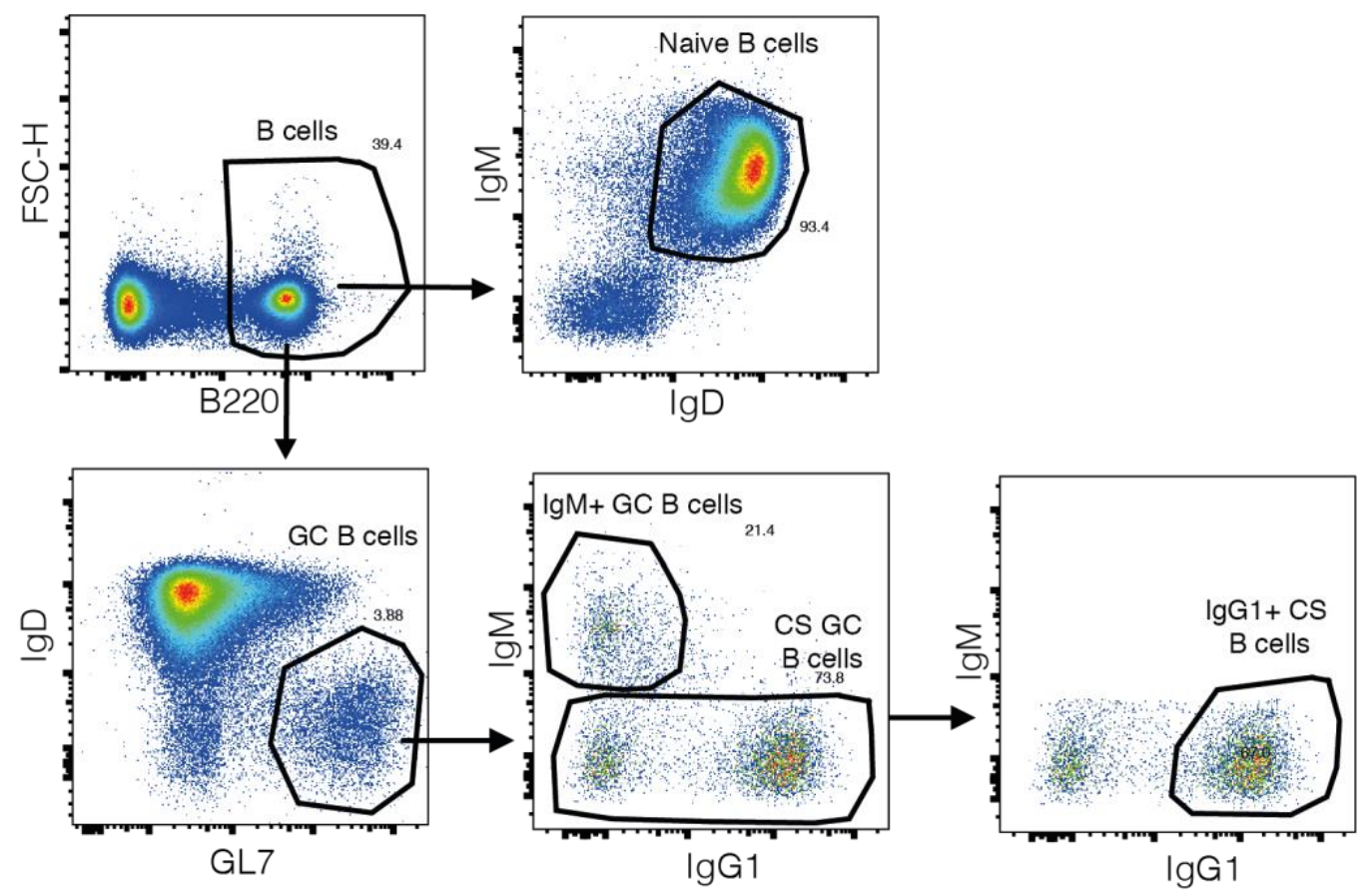

B) Plasma cells

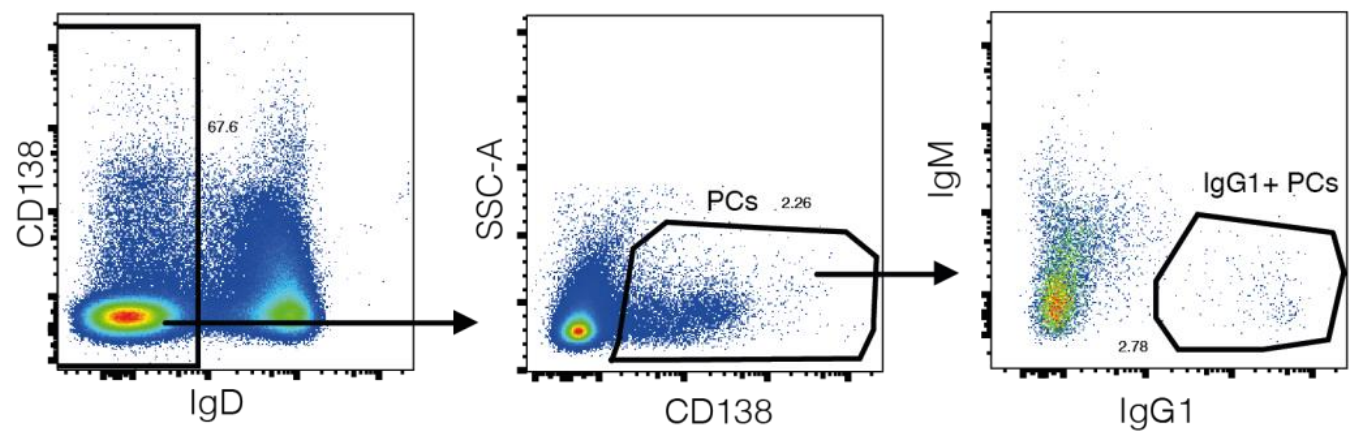

Figure 3.3: Representative flow cytometry gating strategy for $B$ cell and PC populations in the LNs.Gating strategy used to identify (A) B cell populations in the LNs including B cells, naïve B cells, GC B cells, IgM+ GC B cells, CS GC B cells and IgG1+ GC B cells and (B) PC populations in the LNs including PCs and IgG1+ PCs. Pre-gated on live, single cells of interest and arrows indicate parental gate. 


\subsubsection{Antibiotic treatment impairs B cell differentiation}

To determine whether GC development is impaired in ABX-treated, mice were treated with ABX (Figure 3.4A) and the frequencies of B cells (Figure 3.3A) and PCs (Figure 3.3B) in the dLN at 14 days post-TIV were assessed by flow cytometry.

In this experiment, the cellularity of the dLNs did not increase following vaccination, however, $\mathrm{ABX}$ treatment resulted in a significant reduction in the overall cellularity of the dLN of vaccinated mice compared to $\mathrm{H}_{2} \mathrm{O}$ controls (Figure 3.4B). Despite this, total B cells were not significantly reduced in frequency or number in ABX-treated mice (Figure 3.4C). While the frequency of B cells differentiating into GC B cells was not significantly reduced in ABX mice, the overall number of GC B cells were reduced (Figure 3.4D). The frequency and number of GC B cells of the IgM isotype that had not undergone CSR were comparable between $\mathrm{ABX}$ and control vaccinated mice (Figure 3.4E). However, the frequency of GC B cells that had downregulated IgM, thereby undergoing CSR, was reduced in ABX mice and this translated to a significant reduction in the numbers of CS GC B cells (Figure 3.4F). Of cells that had undergone CSR, there were comparable frequencies of the IgG1 isotype following ABX treatment, however the overall number of IgG1 ${ }^{+}$GC B cells was diminished (Figure 3.4G). Strikingly, the frequency and number of PCs in ABX-treated mice was significantly reduced, to levels below PBS vaccinated mice (Figure 3.4H). A comparable frequency of PCs were of the IgG1 isotype but total number of IgG1-secreting PCs was reduced in ABX-treated compared to $\mathrm{H}_{2} \mathrm{O}$ control mice (Figure 3.4I).

Collectively, this analysis shows that CS GC B cells and PC frequencies were reduced following $\mathrm{ABX}$, and this resulted in the number of $\mathrm{IgG1}{ }^{+}$PCs being 
significantly reduced in number. This suggests that altering gut microbial signals impacts multiple stages of B cell development following vaccination with TIV, leading to fewer antibody-secreting PCs and the observed reduction in serum IgG1 antibody. 
(A)

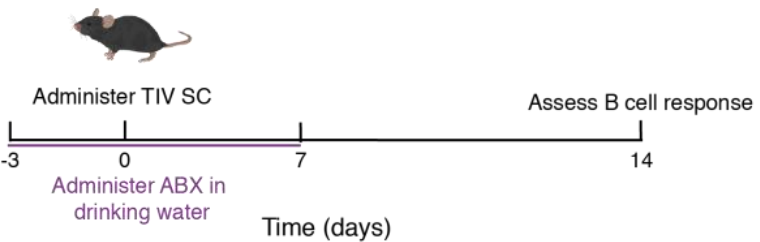

(B)

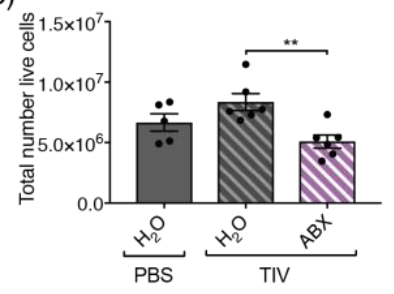

(D)

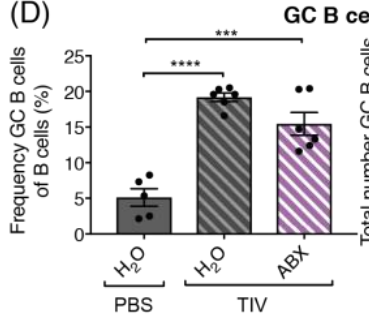

(F) cells

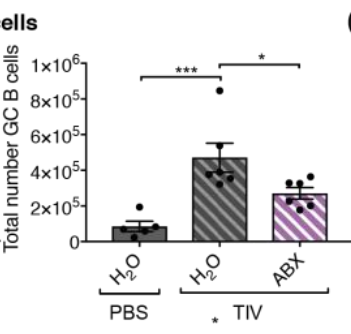

(C)

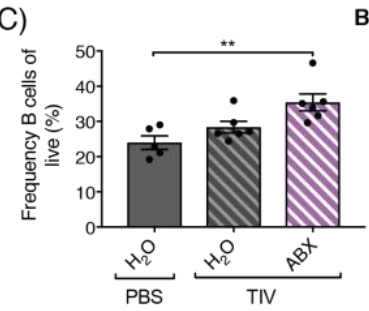

B cells

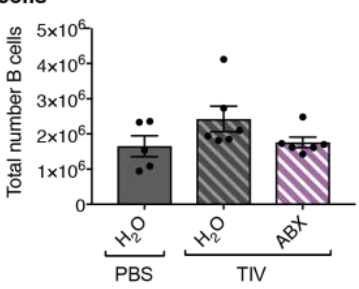

(E)

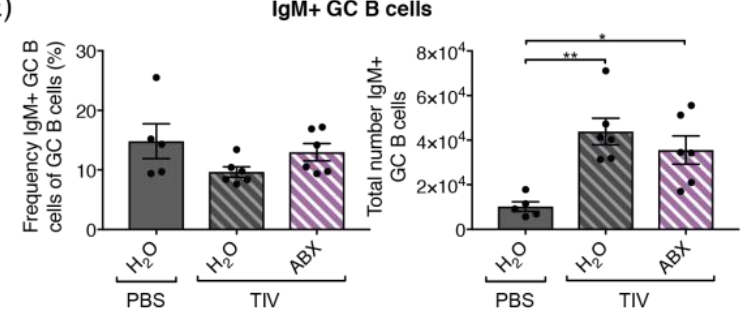

(G)
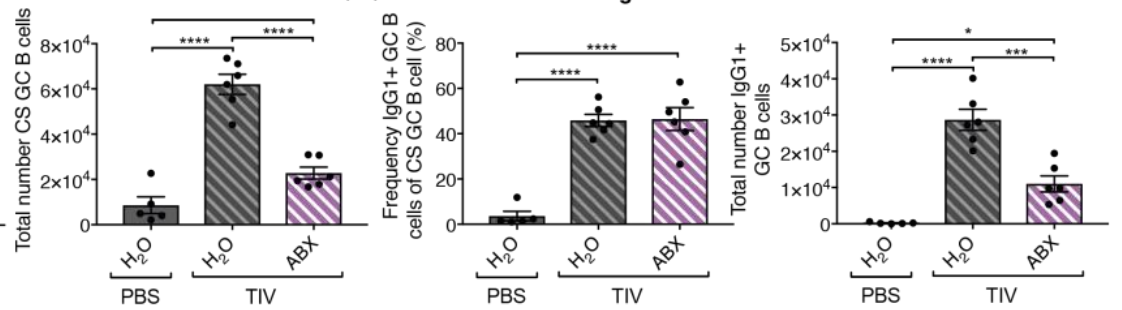

(l)

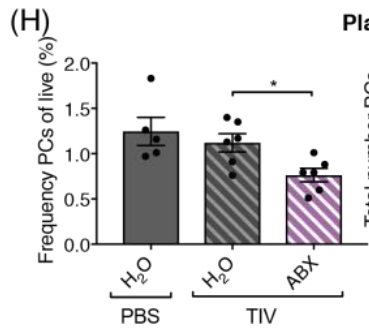

Plasma cells
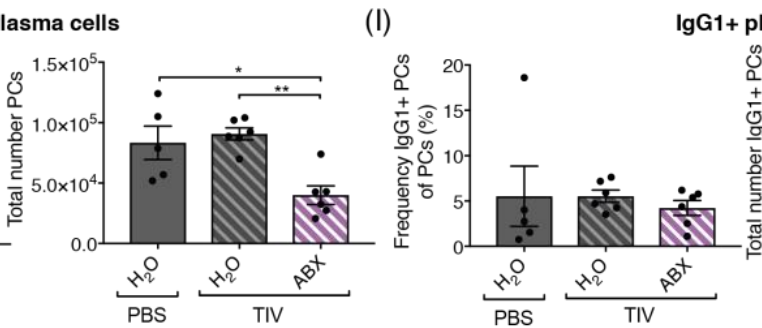

lgG1+ plasma cells

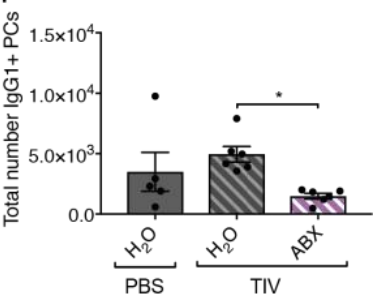

Figure 3.4: Antibiotic treatment impairs multiple stages of B cell differentiation following TIV vaccination. (A) Experimental outline for short-term administration of oral antibiotics and TIV vaccination. $\mathrm{C} 57 \mathrm{BL} / 6$ mice received $\mathrm{ABX}$ or $\mathrm{H}_{2} \mathrm{O}$ ad libitum for three days prior to vaccination until seven days following vaccination. Mice were vaccinated with $200 \mu \mathrm{L} 25 \%$ TIV in PBS or PBS alone SC at the tail base. At 14 days following vaccination, proportion and total number of (E) B cells, (F) GC B cells, (G) IgG1+ GC B cells, (H) PCs and (I) IgG1+ PCs in the inguinal or axillary LNs of mice were determined by flow cytometry analysis. Data shown is $n=5-6$ per group and is representative of three independent experiments. Statistical analysis of flow cytometry data was performed using One-Way ANOVA with Tukey's post-test showing mean \pm SEM. ${ }^{*} \mathrm{p} \leq 0.05,{ }^{* *} \mathrm{p} \leq 0.01,{ }^{* * *} \mathrm{p} \leq 0.001$. 


\subsubsection{Antibiotic treatment reduces follicular helper cell population}

Given the importance of $\mathrm{T}_{\mathrm{FH}}$ derived signals in development and activity of GC $B$ cells, it was hypothesised that $A B X$ treatment may be leading to defective $T_{F H}$ cell help to B cells. Therefore, the frequency of TFH cells in the dLN of ABX-treated mice was assessed by flow cytometry at 14 days post-vaccination (Figures 3.5 and 3.6A).

As was observed in the previous experiment, vaccination did not increase cellularity of the dLN of $\mathrm{H}_{2} \mathrm{O}$ controls compared to naïve mice, however the dLN of $\mathrm{ABX}$-treated mice were significantly reduced in cellularity (Figure 3.6B). The frequency of $\mathrm{CD}^{+} \mathrm{T}$ cells of live cells in the dLN was not altered by vaccination and there was no significant difference in the frequency or number of $\mathrm{CD}^{+} \mathrm{T}$ cells observed between ABX-treated and untreated vaccinated mice (Figure 3.6C). Following vaccination, there was a significant increase in the frequency and number of $\mathrm{T}_{\mathrm{FH}}$ phenotype $\mathrm{CD}^{+} \mathrm{T}$ cells (Figure 3.6D). There was a trend to lower frequencies of $\mathrm{T}_{\mathrm{FH}}$ cells in $\mathrm{ABX}$-treated mice compared to untreated controls, however the number of $\mathrm{T}_{\mathrm{FH}}$ was significantly reduced (Figure 3.6D).

These data demonstrate that depletion of the microbiota with $\mathrm{ABX}$ reduces the number of $\mathrm{T}_{\mathrm{FH}}$ cells in the dLN of TIV vaccinated mice, indistinguishable from baseline numbers, which may be a contributing factor to defects observed in B cell differentiation following vaccination. 


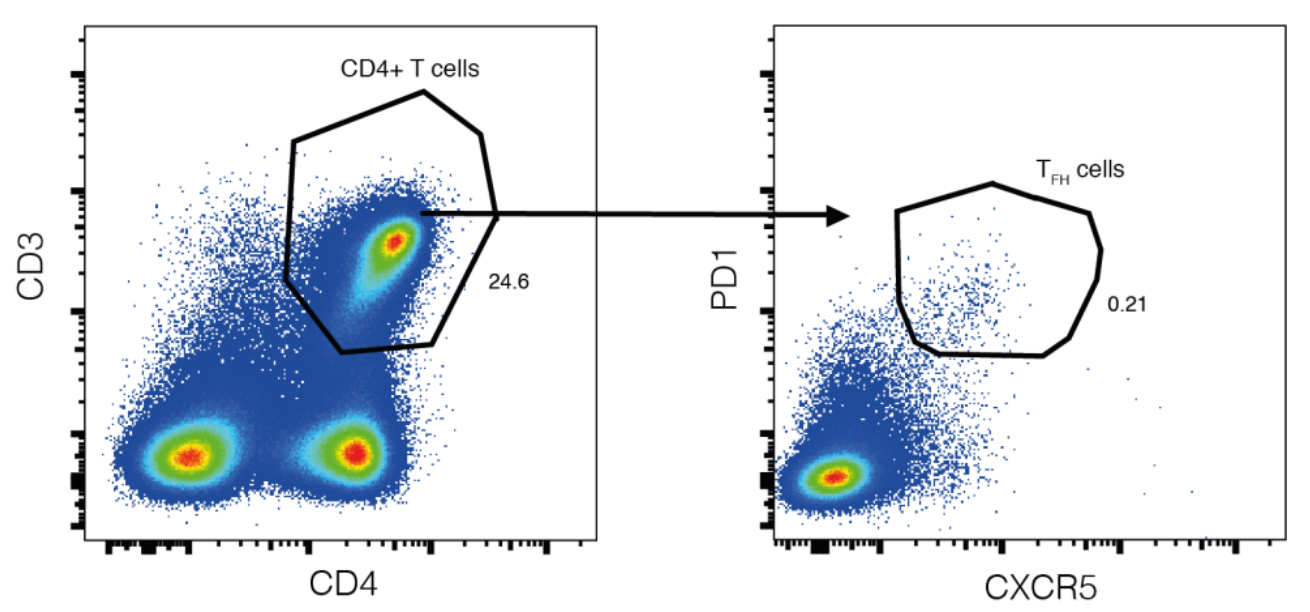

Figure 3.5: Representative flow cytometry gating strategy for $\mathrm{T}_{\mathrm{FH}}$ cells in the LNs. Gating strategy used to identify CD4+ $\mathrm{T}$ cells $\left(\mathrm{CD}^{+}, \mathrm{CD} 4{ }^{+}\right)$and $\mathrm{T}_{\mathrm{FH}}$ cells $\left(\mathrm{CD}^{+}, \mathrm{CD}^{+}, \mathrm{CXCR}^{+}, \mathrm{PD}^{+}\right)$. Gated on live, single cells of interest and arrows indicate parental gate. 
(A)
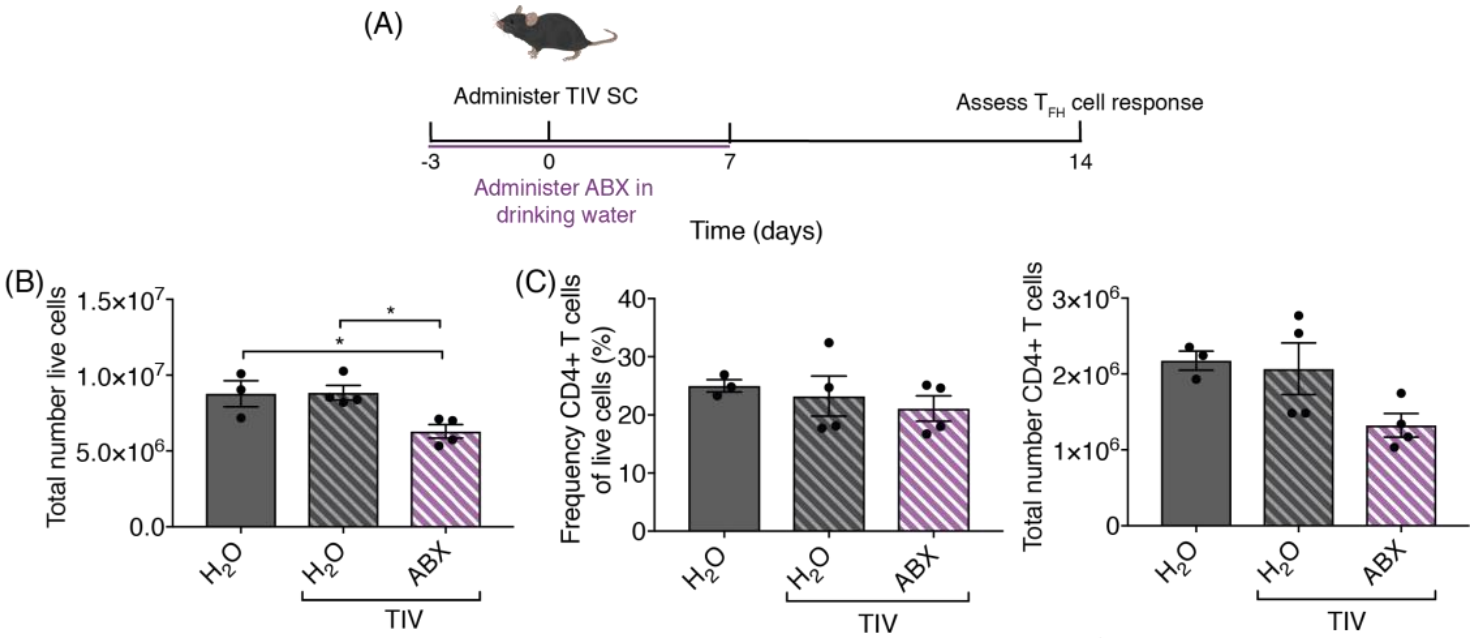

(D)
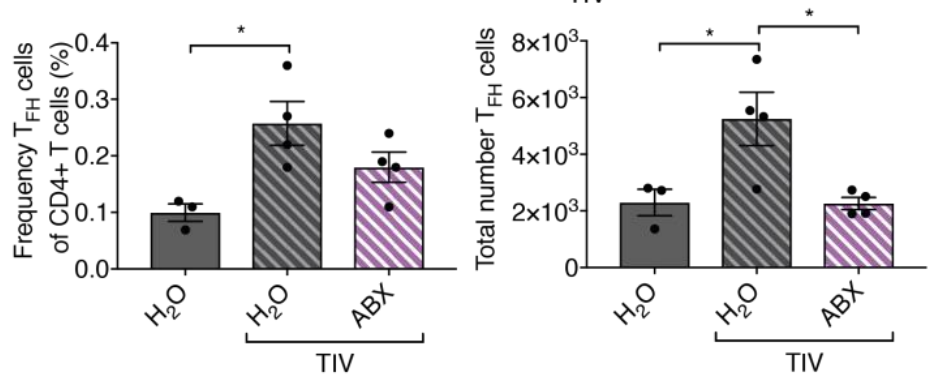

Figure 3.6: Antibiotic treatment leads to a reduction in $\mathrm{T}_{\mathrm{FH}}$ cells in the $\mathrm{dLN}$ following vaccination. (A) Experimental outline for short-term administration of oral antibiotics and TIV vaccination. $\mathrm{C} 57 \mathrm{BL} / 6$ mice received $\mathrm{ABX}$ or $\mathrm{H}_{2} \mathrm{O}$ ad libitum for three days prior to initial vaccination until seven days following vaccination. Mice were vaccinated SC with $200 \mu \mathrm{L} 25 \%$ TIV in PBS or PBS alone at the tail base. At 14 days following vaccination, (B) total number of live cells in the dLNs, proportion and total number of (C) CD4 $4^{+} \mathrm{T}$ cells and (D) TrH cells were determined by flow cytometry analysis. Data shown is $n=3-4$ per group. Statistical analysis of flow cytometry data was performed using One-Way ANOVA with Tukey's post-test showing mean \pm SEM. ${ }^{*} \mathrm{p} \leq 0.05$. 


\subsubsection{AF488 labelled TIV vaccine fluorescently labels cells non-specifically in vivo}

The data presented thus far demonstrates that modifying the gut microbiota composition with oral $\mathrm{ABX}$ throughout the period of TIV vaccination leads to impairments in GC B cell development at multiple stages and a subsequent reduction in vaccine-specific antibody production. As DCs and $\mathrm{M} \varphi$ play central roles in both $\mathrm{T}$ and $\mathrm{B}$ cell activation and differentiation in the $\mathrm{dLN}^{62}$, microbiota alterations may be impacting the frequency or activation status of myeloid cell subsets, affecting their ability to transport and present antigen, resulting in defects in B and TrH cell activity.

TIV vaccine antigen was labelled with AF488 fluorescent dye in order to track myeloid subsets that take up vaccine antigen and migrate to the dLN. This technique is routinely utilised at MIMR to track antigen uptake by phagocytic cells ${ }^{216}$. Prior to administration, the protein content of AF488-labelled vaccine was equated to that administered in unlabeled TIV vaccination using the bicinchoninic acid (BCA) protein quantification assay. Initially, it had to be confirmed that phagocytic cells could be identified as antigen-positive by flow cytometry in the dLN and it was necessary to ensure labelling the vaccine did not alter the vaccine efficacy. To investigate this, mice were vaccinated with

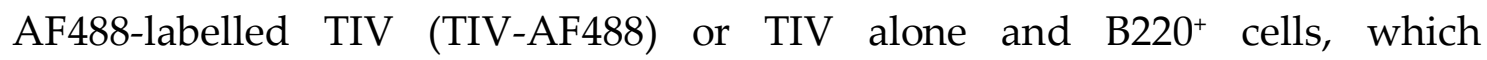
encompasses B cells and pDCs ${ }^{217}$, LNresDCs, MigDCs, M $\varphi$ and T cells were assessed for antigen uptake by flow cytometry analysis 24 or 42 hours later (Figures 4.7 and 4.8A). TIV-AF488 ${ }^{+}$cells were detected at both 24 and 42 hours following vaccination (Figure 3.8B and C). Of cells that were TIV-AF488', significant proportions were B220+ cells, LNresDCs, MigDCs and $\mathrm{M} \varphi$, which are phagocytic and are expected to take up the vaccine. However, 10-20\% of TIV-AF488 ${ }^{+}$cells at 24 and 42 hours post-vaccination (H.P.V.) were T cells (Figure 
3.8D), and not expected to acquire antigen. This data suggests that TIV-AF488 is binding to or being taken up by cells irrespective of their ability to phagocytose large particles. Furthermore, the total number of $\mathrm{B} 220^{+}$cells, MigDCs and $\mathrm{M} \varphi$ were significantly lower following TIV-AF488 compared to TIV vaccination alone (Figure 3.8E), suggesting the immunogenicity of TIV-AF488 is not equal to TIV or that it is somehow leading to loss of cellularity in the LN. Therefore, it will not serve as a reliable way to track TIV antigen uptake in vivo.

The AF488 protein labelling kit used specified purification over a silica column with no subsequent washes. I hypothesised that part of the signal observed in cells other than APCs could be due to non-specific binding of unbound AF488 dye, therefore the vaccine was washed prior to administration. A more extensive range of cell subsets were assessed for antigen uptake 24 H.P.V. by flow cytometry (Figure 3.7 and Figure 3.8F). Importantly, total numbers of all subsets of cells analysed were not significantly different between mice vaccinated with TIV-AF488 and TIV alone (Figure 3.8J), suggesting that washing rectified the issue of differential immunogenicity. However, only a small proportion of live cells could be identified as being TIV-AF488 ${ }^{+}$, and was not statistically greater than levels as compared to TIV alone (Figure 3.8G and H). Furthermore, even after extensive washing, a large proportion of $\mathrm{AF} 488^{+}$cells were $\mathrm{T}$ cells (Figure 3.8I). This demonstrates that even with extensive washing the TIV-AF488 labels non-phagocytic cells and therefore will not be suitable to track TIV vaccine uptake in vivo. 
(A)
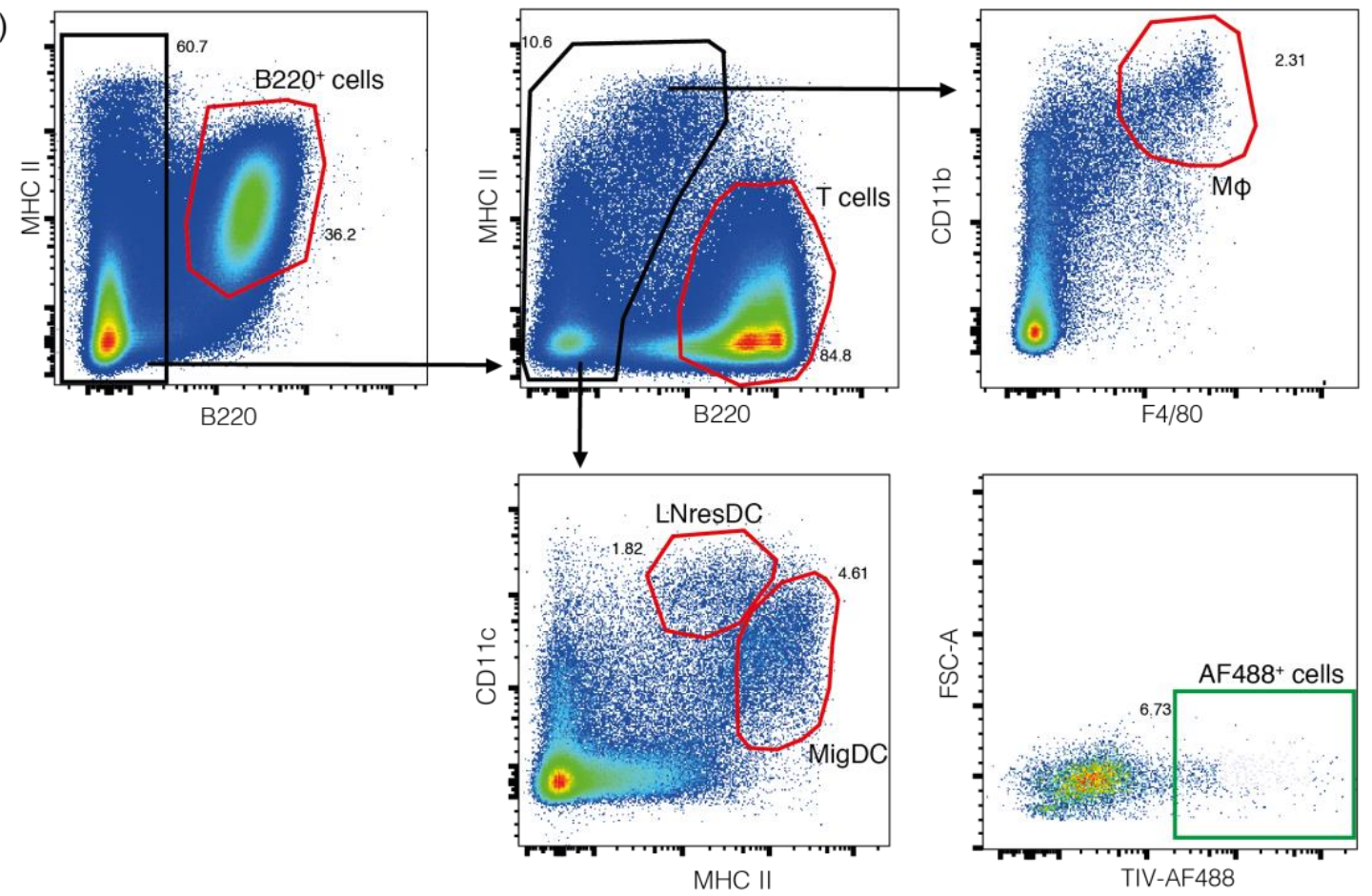

(B)
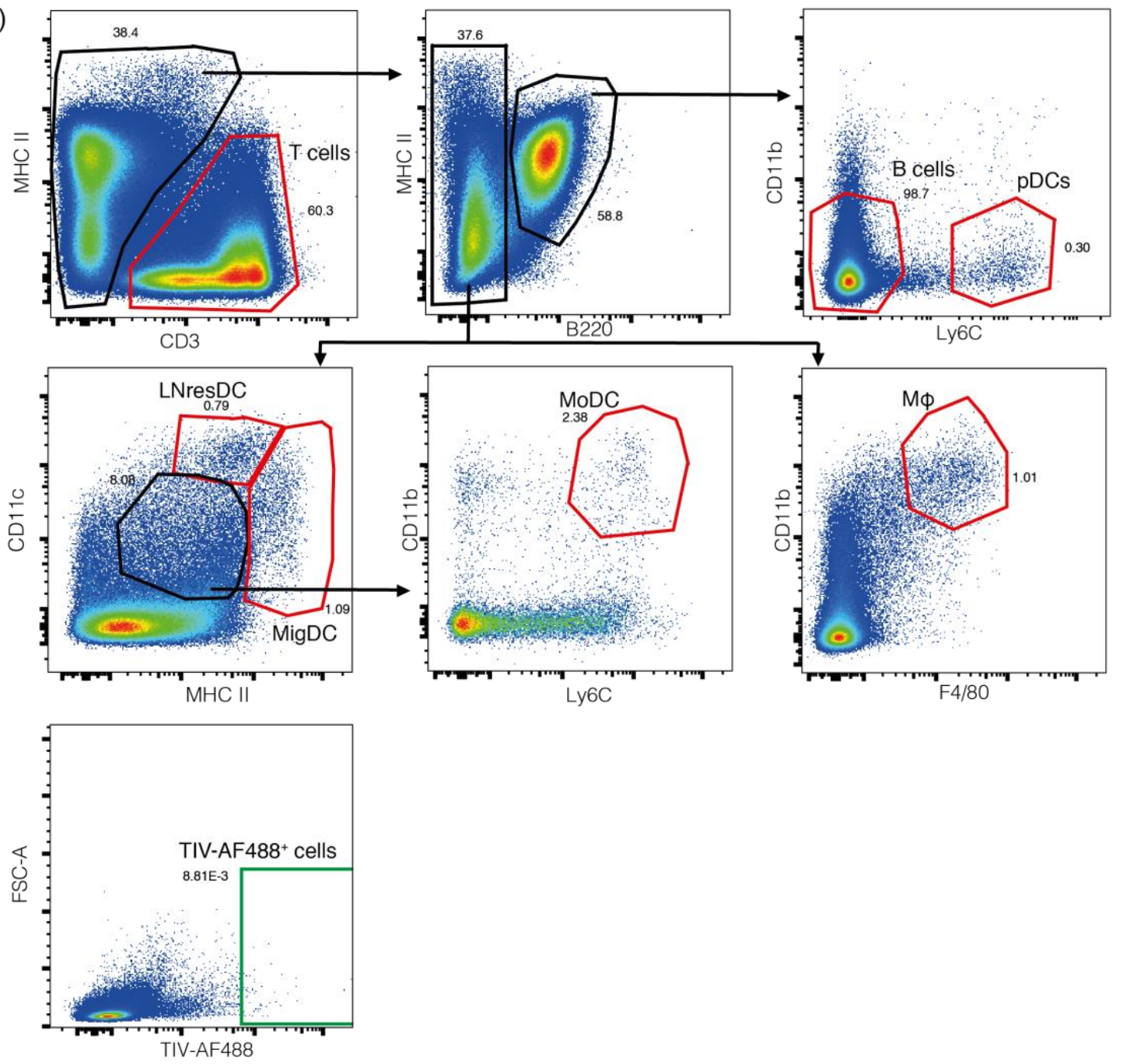
Figure 3.7: Representative flow cytometry gating strategy for vaccine-labelled cells in the dLN. Gating strategies used in analysis of cell subsets after administration of TIV-AF488 purified without (A) and with (B) subsequent washing B220+ cells, T cells (CD3+), LNresDCs (CD3-, B220-,

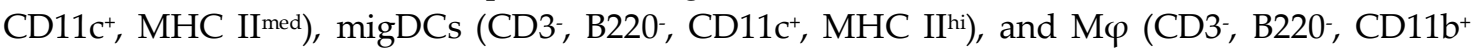
$\mathrm{F} 4 / 80^{+}$). From each cell subset (red gates) the frequency of TIV-AF488 cells were determined (green gate). Pre-gated on live, single cells of interest. Arrows indicate parental gate. 
(A)

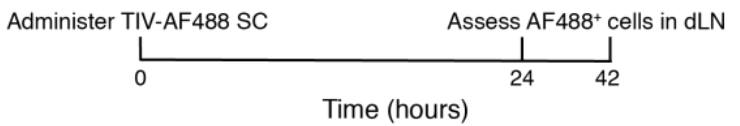

(B)

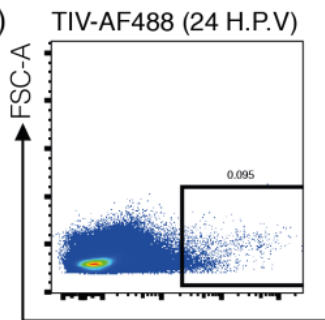

(D)

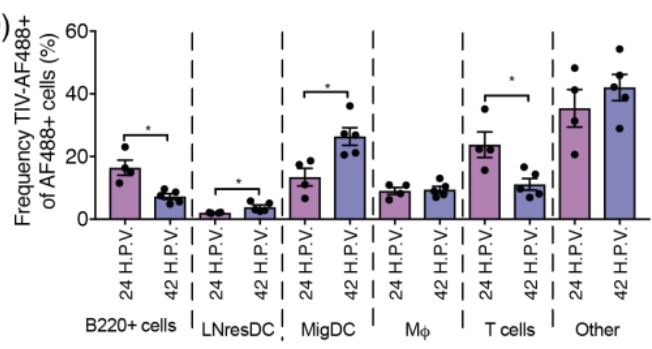

(F)
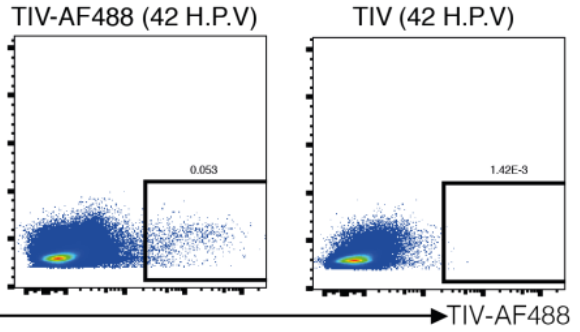

(C)

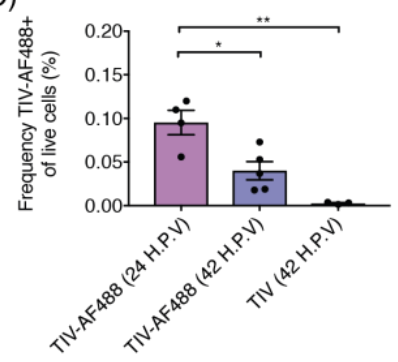

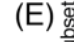

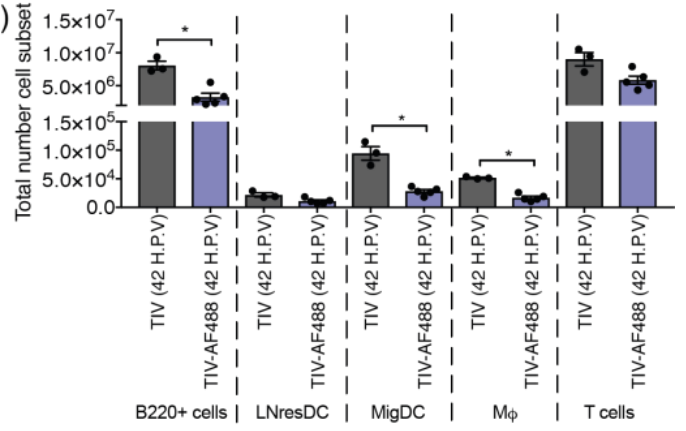

(G)

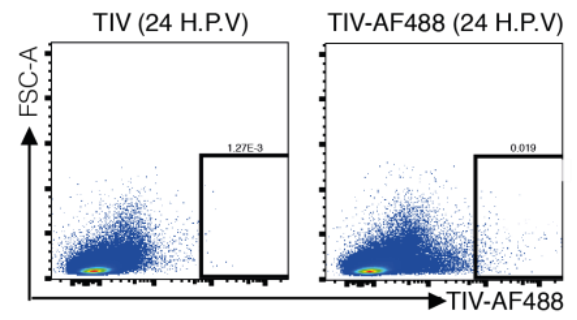

(I) Frequency cell subset of $\mathrm{AF} 488^{+}$cells (24 H.P.V)

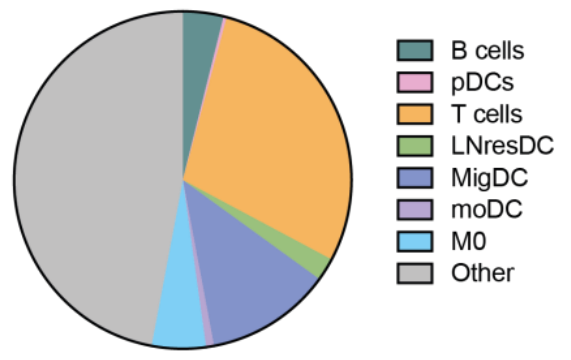

(H)
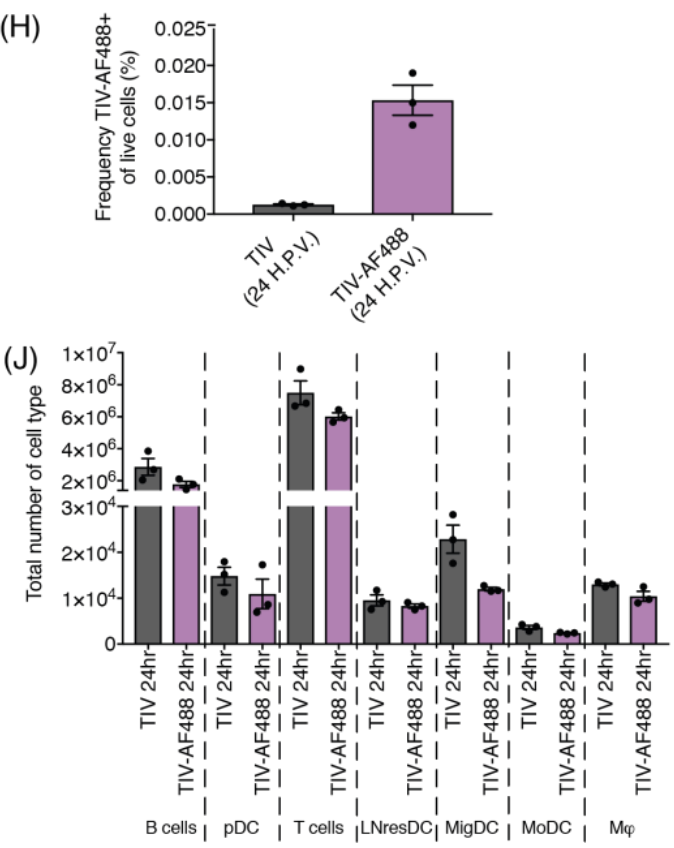
Figure 3.8: Fluorescently labelled TIV vaccine is acquired by non-APC populations. (A) Experimental outline for vaccination with AF488 labelled TIV vaccine (TIV-AF488). C57BL/6 mice were administered TIV-AF488 or unlabeled TIV and numbers and frequencies of AF488 $\mathrm{B} 220^{+}$cells, T cells, migDCs and M $\varphi$ were assessed in the dLN at 24 or 42 hours post-vaccination (H.P.V.) by flow cytometry analysis. (B) Plots showing TIV-AF488 uptake among live cells. (C) Frequency of TIV-AF488 $8^{+}$cells among live cells. (D) Proportion of cell subsets that are TIV-AF488 ${ }^{+}$ among total TIV-AF488 ${ }^{+}$cells. (E) Total numbers of cell subsets. Graphs show mean \pm SEM. Data shown is n=3-6 per group. Statistical significance of $(\mathbf{C})$ was determined by One-way ANOVA with Tukey's post-test and for (D), (E), (H) and (J) was determined by Mann-Whitney test. ${ }^{*} \mathrm{p} \leq 0.05,{ }^{* *} \mathrm{p} \leq 0.01$. 


\subsubsection{Antibiotic treatment impacts number and activation status of myeloid cells at steady-state}

Unfortunately, the AF488-labelled TIV could not be used to track myeloid cells capability to transport TIV to the dLN due to non-specific labelling. To assess impact of $\mathrm{ABX}$ treatment on myeloid responses, instead changes in overall myeloid populations in the dLN in ABX-treated mice were analysed. Mice were administered $\mathrm{ABX}$ and myeloid populations assessed at steady-state to assess myeloid population status at time of vaccine antigen encounter (Figure 3.9 and 4.10A).

Three days of ABX treatment led to significant reduction in the cellularity of the dLN (Figure 3.10B). Of these live cells, there were no significant changes in the frequency of pDCs or LNresDCs (Figure 3.10C). MigDCs represented a higher proportion of the live cells in the LN in ABX-treated mice, whereas the frequency of MoDCs and M $\varphi$ were both significantly reduced in these mice (Figure 3.10C). There were comparable numbers of LNresDCs and MigDCs between mice administered water and $A B X$, whereas $A B X$ mice had significantly fewer MoDCs and $\mathrm{M} \varphi$, and a trend to fewer pDCs (Figure 3.10D). The expression of the costimulatory molecule, CD86, was reduced in pDCs, LNresDCs and MigDCs, however without statistical significance.

Collectively, these data demonstrate that $\mathrm{ABX}$ treatment in the three days leading up to vaccination impacts myeloid populations in the vaccine-draining $\mathrm{LN}$, reducing overall frequencies and expression of costimulatory molecules. 


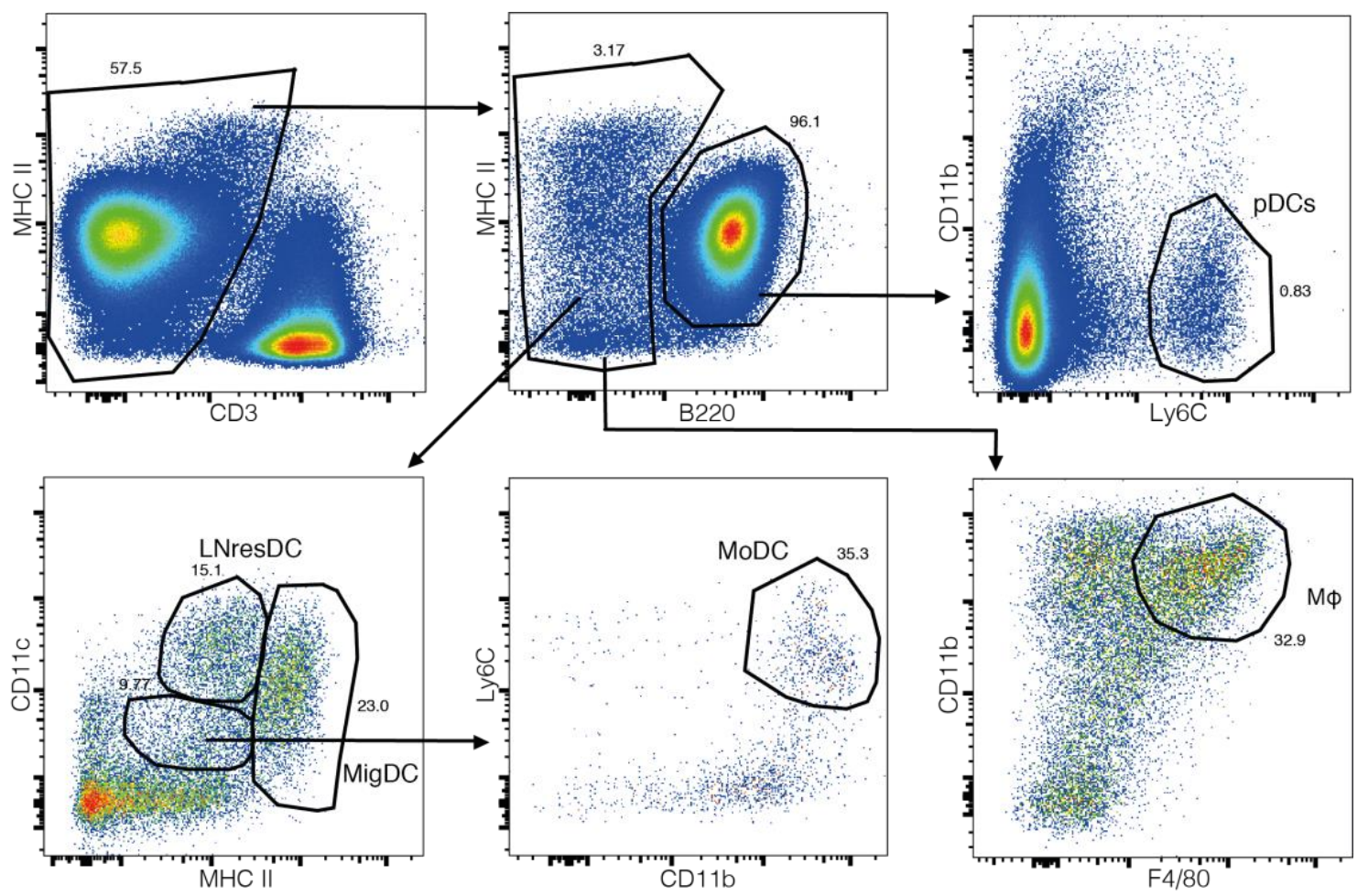

Figure 3.9: Representative flow cytometry gating strategy for myeloid cells in the LNs. Gating strategy used to identify pDCs (CD3-, B220+, CD11b-, Ly6C+) LNresDCs (CD3-, B220-, CD11 ${ }^{+}$ MHC II $\left.{ }^{\mathrm{med}}\right)$, migDCs (CD3-, B220-, CD11 $\mathrm{c}^{+}$, MHC II $\left.{ }^{\mathrm{hi}}\right)$, moDCs (CD3-, B220-, CD11 $\mathrm{c}^{\mathrm{med}}$, MHC II ${ }^{\mathrm{med}}$

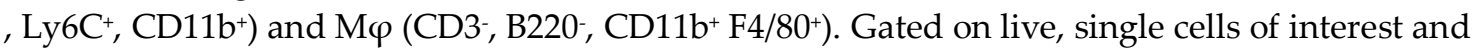
arrows indicate parental gate. 
(A)

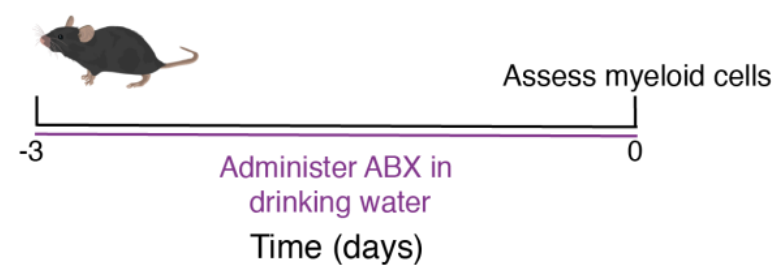

(B)

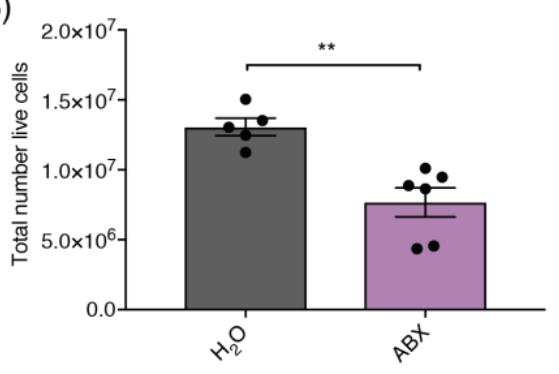

(D)

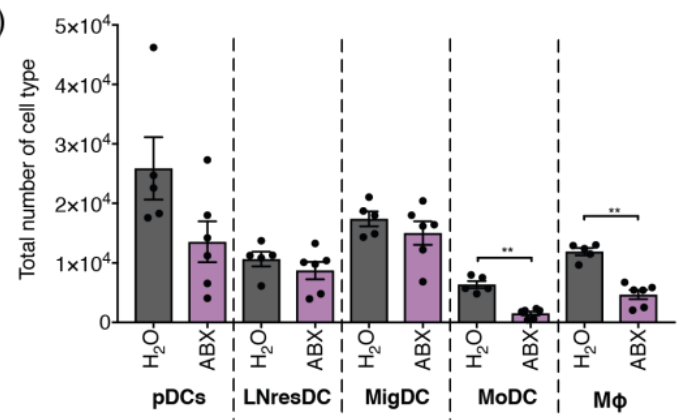

(C)

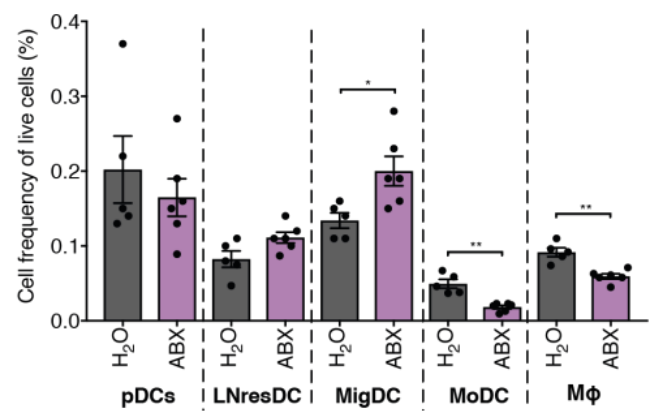

(E)

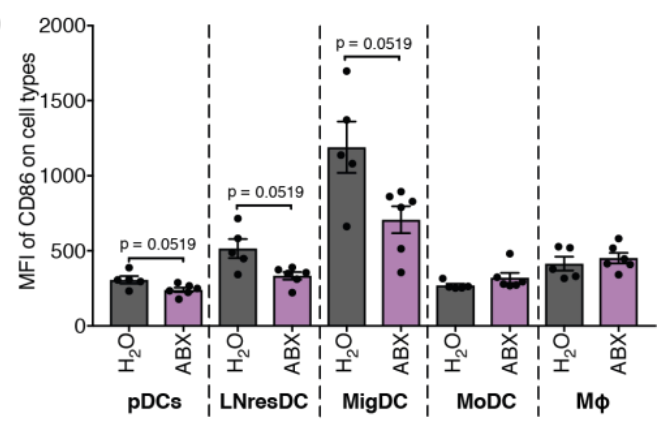

Figure 3.10: Antibiotic treatment decreases myeloid cell numbers and activation state. (A) Experimental outline for short-term administration of oral antibiotics. C57BL/6 mice were administered $\mathrm{ABX}$ or $\mathrm{H}_{2} \mathrm{O}$ ad libitum for three days. Following $\mathrm{ABX}$ treatment, (B) the total number of live cells in the dLNs, (C) proportions of cell subsets within live cells, (D) total number of cell subsets and (E) CD86 MFI were determined by flow cytometry analysis for pDCs, LNresDCs, migDCs, moDCs and M $\varphi$. Data shown is $n=5-6$ per group and representative of two independent experiments. Statistical analysis was performed using Mann-Whitney test showing mean \pm SEM. ${ }^{*} \mathrm{p} \leq 0.05,{ }^{* *} \mathrm{p} \leq 0.01$. 


\subsubsection{Antibiotic treatment impacts number and activation status of myeloid cells following vaccination}

Next, to determine if similar impairments may be seen following vaccination, mice were administered $\mathrm{ABX}$ and sacrificed on day three following TIV vaccination for flow cytometry analysis of myeloid cell populations (Figure 3.9 and 3.11A).

A striking reduction in LN cellularity was observed in mice treated with $A B X$ (Figure 3.11B) and significantly reduced frequency of pDCs, LNresDCs, MoDCs and $\mathrm{M} \varphi$ was observed, however the frequency of migDCs were not significantly reduced (Figure 3.11C). Reduced overall cellularity coupled with stark reductions in frequency of myeloid cells translated to severely reduced numbers of all myeloid populations analysed (Figure 3.11D). Furthermore, all myeloid populations analysed, excluding MoDCs, had diminished expression of costimulatory molecule CD86 (Figure 3.11E).

Collectively, these data suggest that altering microbiota-derived signals by oral $\mathrm{ABX}$ treatment is detrimental to the frequency and activation status of myeloid populations under homeostatic conditions and following vaccination. 
(A)

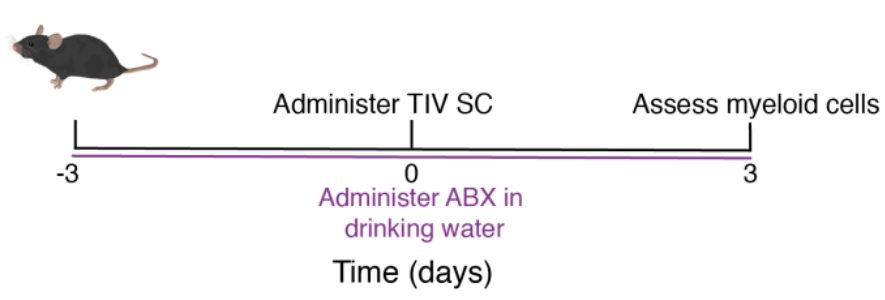

(B)
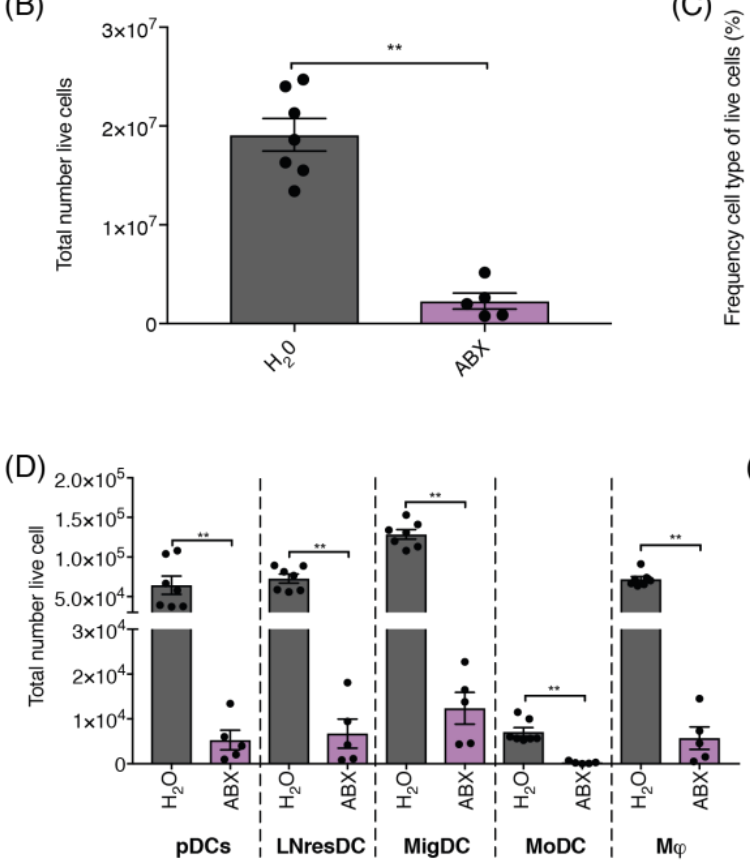

(C)

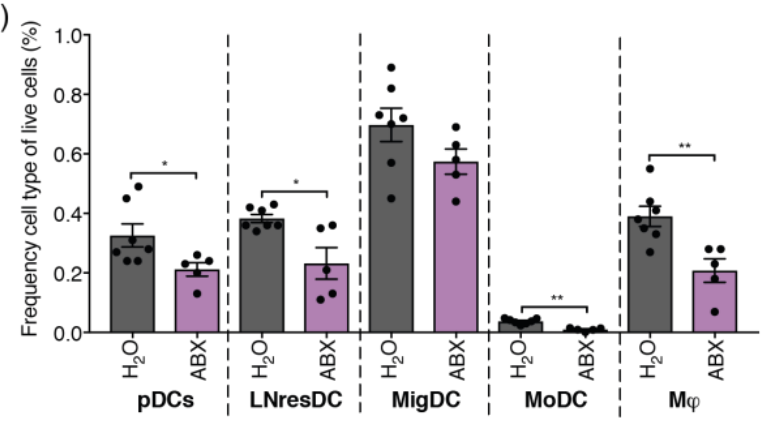

(E)

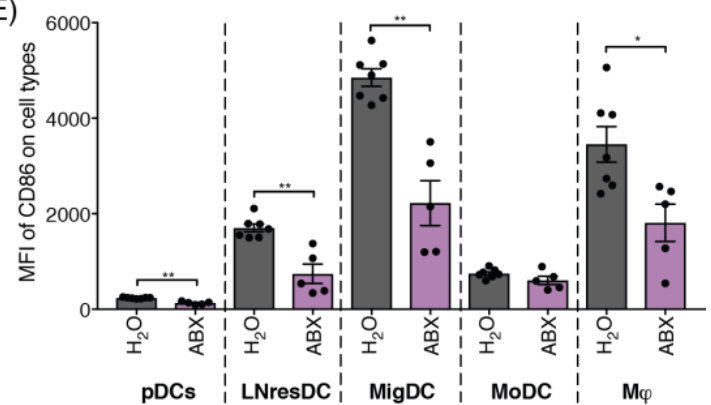

Figure 3.11: Antibiotic treatment severely impacts myeloid cell frequencies and activation status following TIV vaccination. (A) Experimental outline for short-term administration of oral antibiotics and TIV vaccination. C57BL/6 mice were administered $\mathrm{ABX}$ or $\mathrm{H}_{2} \mathrm{O}$ ad libitum for three days prior to vaccination until sacrificed three days post-vaccination. Three days following vaccination (B) the total number of live cells in the dLNs, (C) proportions of cell subsets within live cells, (D) total number of cell subsets and (E) CD86 MFI on pDCs, LNresDCs, migDCs, moDCs and $\mathrm{M} \varphi$ was analysed by flow cytometry analysis. Data shown is $n=5-7$ per group and representative of two independent experiments. Statistical analysis was performed using MannWhitney test showing mean \pm SEM. ${ }^{*} \mathrm{p} \leq 0.05,{ }^{* *} \mathrm{p} \leq 0.01$. 


\subsection{Discussion}

Previous investigations have shown that microbiota-derived flagellin has an adjuvant effect in TIV vaccination, providing a critical signal to $\mathrm{M} \varphi$ and $\mathrm{B}$ cells and is required to induce robust antibody responses ${ }^{72}$. The aim of this chapter was to further assess the impact of the intestinal microbiota on the humoral immune response to TIV vaccination in mice. Broad-spectrum ABX were administered to alter gut microbial communities in order to investigate the impact of the gut microbiota on the humoral immune response to TIV vaccination. A ten-day course of oral $A B X$ was sufficient to alter gut microbial communities and severely impair vaccine-specific antibody production. Concurrently, ABX treatment impaired development of the dLN B cell response at multiple stages in the dLN following TIV vaccination. $T_{\mathrm{FH}}$ populations were also reduced in number in ABX-treated mice. TIV vaccine antigen was labelled to allow for tracking of antigen uptake by myeloid populations, However, this technique was not able to be optimised. Instead, total myeloid cell populations in the dLN were assessed. Multiple populations were found to be significantly reduced in number, as well as exhibiting a less activated phenotype, at both steady-state and post-vaccination in ABX-treated mice compared to untreated controls.

A ten-day course of broad spectrum $A B X$ recapitulated the detrimental effect on antibody production observed with a six-week regime without the confounding factor of dehydration, and therefore this regime was employed. It was confirmed that $\mathrm{ABX}$ treatment significantly altered microbial phyla composition. Ideally, this analysis of bacterial phyla in the faeces would have been repeated in a further experiment but unfortunately the extracted DNA was of poor quality. Faecal samples from mice after both three and ten days of ABX treatment had 
very low A260/A230 ratios by spectrophotometric analysis, indicating a high ratio of organic contaminants to DNA. This is likely due to a reduction in total quantity of faecal bacterial DNA following ABX treatment. Although it was not determined whether bacterial load was reduced by additional methods, other investigators observed a greater than two log reduction in the faecal bacterial load with a similar ABX regime ${ }^{71}$. These results suggest bacterial load was substantially reduced which, in turn, would restrict host circulating levels of microbiota-derived immunostimulatory PAMPs and bacterial metabolites and is likely a key factor in the effect of ABX treatment on capability to mount anti-TIV humoral response.

At 14 days post-vaccination, B cells in the dLN were analysed to determine whether $A B X$ impaired any stage in the generation of the $B$ cell response. $A$ major finding was the reduction in the frequency of GC B cells that had undergone CSR in ABX-treated mice. Additionally, the frequency of cells differentiating into PCs was also reduced. Of note, defects were not universal across all B cell populations, as the number and frequency of total B cells and non-class-switched B cells were comparable in vaccinated mice following ABX treatment. The effect of $\mathrm{ABX}$ on multiple stages of $\mathrm{B}$ cell development culminated in significantly fewer IgG1 ${ }^{+}$PCs being produced, correlating with reduced serum IgG1 antibody titres.

B cell development is a collaborative effort, relying on concerted action between many different cells. Based on the findings in B cells, other cells crucial to GC B cell and plasma cell development were subsequently analysed. In the GC, TFH cells provide important signals to aid B cell activation, proliferation, differentiation and survival ${ }^{73,74}$. Signals include $\mathrm{T}_{\mathrm{FH}}$ cell CD40L binding to CD40 expressed by B cells, which is a critical signal for multiple stages of GC B cell activation, differentiation and cytokine secretion such as IL-21 which potently 
promotes GC B cell CSR and PC differentiation ${ }^{75}$. Whilst the frequency of CD4 ${ }^{+}$ $\mathrm{T}$ cells of the $\mathrm{T}_{\mathrm{FH}}$ phenotype were comparable between $\mathrm{ABX}$ and water-administered groups, the total number of $\mathrm{T}_{\mathrm{FH}}$ cells following vaccination in ABX-treated mice was significantly reduced. This may contribute to the impaired B cell responsiveness observed in ABX-treated mice, as GC B cell competition for $T_{F H}$ cell help is already a response-limiting factor ${ }^{76}$. However, as GC B cells in the dLN are also significantly reduced in number, the ratio of GC B cells to TFH cells may not be different, and in this case competition for $\mathrm{T}_{\mathrm{FH}}$ cell help would also remain constant. Further investigation is required to determine if the reduced number of $T_{F H}$ cells is having a causative effect in defective B cell development.

Impaired development of the $\mathrm{B}$ cell and $\mathrm{T}_{\mathrm{FH}}$ cell response was observed in ABX-treated mice, both of which require signals from myeloid populations for priming and differentiation. In order to determine if myeloid subsets that take up the vaccine antigen and migrate to the dLN were impaired following $A B X$ treatment, TIV vaccine antigen was labelled with AF488 fluorescent dye. Unexpectedly, a proportion of $\mathrm{T}$ cells stained positively for AF488. Antigen uptake should be restricted to phagocytic cells and as T cells are non-phagocytic, this indicated cells were non-specifically acquiring antigen. This may be due to labelled HA protein in the vaccine binding the surface of cells via sialic acid residues, which are ubiquitously expressed on the surface of all cells ${ }^{77}$, including T cells ${ }^{218}$. This is supported by studies utilising a labelled HA probe to identify antigen-specific B cells following influenza infection, finding it was necessary to cleave sialic acid resides by pre-treating cells with NA prior to staining in order to prevent non-specific binding ${ }^{219}$. Furthermore, only a small proportion of myeloid cells were antigen positive following vaccination and the inability to distinguish $\mathrm{AF}_{48} 8^{+}$myeloid populations was exacerbated by the high level of 
cellular autofluorescence in the AF488 detector. Following vaccination with TIV-AF488, an increased number of events could be observed amongst the background autofluorescence - these are likely cells that have taken up low levels of antigen - resulting in the true frequency of $\mathrm{AF} 488^{+}$cells being underrepresented. The AF488 detector has a bandpass filter with a range that optimally picks up cellular autofluorescence (505nm-525nm $)^{220}$. Therefore, in future investigations, protein should be labelled with a fluorescent probe that does not fluoresce at a similar wavelength to autofluorescent cells. Due to the low frequency of antigen-positive events, the frequency of antigen positive myeloid cells in these mice is likely to be challenging to quantify. For these reasons, it was decided the labelled vaccine could not be reliably used to track vaccine uptake.

Tissue-resident myeloid populations are the first cells that encounter antigen following vaccination. It would be interesting to determine what effect $A B X$ had on these cells prior to vaccination, which would likely affect their ability to take up vaccine antigen, migrate to the dLN and prime adaptive immune responses. However, due to time constraints this protocol could not be developed. Instead, steady-state LN-resident myeloid cells were analysed as these are also likely to play a key role in initial priming immunity and $B$ cell responses in TIV vaccination. Small soluble antigens freely drain from the site of SC vaccination to be acquired by LNresDCs, independently of transport by migDCs, as demonstrated by the observation that DCs acquire antigen in the dLN by three hours after SC injection ${ }^{36,38}$, while it takes around 12 hours for migDCs to arrive in the $\mathrm{LN}$ from the tissue ${ }^{39}$. Following three days of ABX treatment and prior to vaccination, cellularity of the dLN was significantly reduced. Interestingly, ABX treatment had differential effects on myeloid cell subsets, significantly reducing the number and frequency of MoDCs and $M \varphi$, however, not impacting their 
expression of costimulatory marker CD86. Conversely, the number and frequency of other DC populations remained unaffected, yet they consistently expressed lower levels of CD86. It should be noted myeloid populations have different developmental origins: the pDCs and classical DCs (cDCs), which encompass migDCs and LNresDCs, develop from a common BM-derived DC precursor $^{221,222}$. In contrast, moDCs differentiate from circulating monocytes that migrate from the blood in response to microbial and inflammatory stimuli ${ }^{223}$, while $\mathrm{M} \varphi$ can be also be derived either from circulating monocytes ${ }^{224}$ or embryonic progenitors that are self-renewed in the periphery ${ }^{225,226}$. In the absence of certain microbial signals with $\mathrm{ABX}$ treatment, it is feasible that replenishment of MoDCs or $\mathrm{M} \varphi$ in the LN by monocyte differentiation is reduced. In addition, DC and M $\varphi$ subsets exhibit distinct expression patterns of PRRs ${ }^{227}$, therefore responding to different microbial stimuli and consequently would be differentially affected by $\mathrm{ABX}$-induced dysbiosis, potentially leading to a reduced activation phenotype.

The effect of $\mathrm{ABX}$ on activity of myeloid populations was further demonstrated at three days following vaccination. Further reductions were observed in dLN cellularity, with all $\mathrm{M} \varphi$ and DC subsets drastically reduced in number. Expression of CD86 was reduced on all myeloid subsets with the exception of MoDCs, in contrast to what was observed at steady-state. Due to a general reduction in all myeloid cell subsets, it may be that a lack of microbiota signals is altering cellular trafficking to the peripheral LN to replenish or sustain myeloid cells here. Indeed, the microbiota controls pDC trafficking by sustaining constitutive levels of CCL2, which is necessary for pDC egress from the BM and their distribution in the blood and peripheral tissues ${ }^{228}$. Additionally, consistent with the role of gut microbiota signalling in maintenance of myeloid progenitor cells of $\mathrm{BM}$ and yolk-sac origin ${ }^{159,229}$, it is also conceivable that defects in 
myelopoiesis may lead to the general reduction in myeloid cell numbers observed both at baseline and post-vaccination. However, previous investigations show defects in myelopoiesis have been restricted to longer durations of $\mathrm{ABX}$ treatment (four to five weeks) and it is known that DCs in LNs are replaced every ten to 14 days ${ }^{230}$, so it remains to be determined if a defect in haematopoesis could lead to reduced numbers of peripheral myeloid populations in just three to six days.

Collectively, these data demonstrate that defects in B cell development could be due to altered signalling of microbial components either directly on B cells or indirectly on cells important to B cell development, such as T cells or myeloid cell subsets. The microbiota may be directly acting on $\mathrm{T}$ cells via TLRs ${ }^{231,232}$. Recently, T cell-intrinsic TLR signalling was shown to promote $\mathrm{T}_{\mathrm{FH}}$ development in the gut and subsequent GC formation and IgA production ${ }^{233}$. Furthermore, the significant reduction in the number of myeloid cells and their activation status in the dLN at steady-state and following vaccination in ABX treatment likely impacts $\mathrm{T}$ cell activation, given that DCs supply cytokines and present antigen required for $\mathrm{T}$ cell priming and differentiation to $\mathrm{T}_{\mathrm{FH}}$ cells $\mathrm{s}^{46,47}$. Fewer myeloid cells with reduced costimulatory capacity may have contributed to the lower number of $\mathrm{T}_{\mathrm{FH}}$ observed following $\mathrm{ABX}$ treatment, thereby indirectly impacting B cell responses. DCs and $\mathrm{M} \varphi$ also present antigen to B cells in the LNs for their initial activation, so may have impacted B cells in this way. Alternately, ABX treatment may have impacted B cells directly. This is supported by previous studies showing that signalling of microbial components through TLRs on B cells is necessary to prompt effective B cell differentiation and antibody production ${ }^{69,158,234}$ and microbially-produced SCFAs directly provide B cells with substrates for metabolism and by inhibiting HDACs 
enhance expression of genes required for PC differentiation that, in turn, boosts antibody production ${ }^{175}$.

\subsection{Conclusion}

In this chapter, it has been demonstrated that short-term administration of broad-spectrum ABX alters the composition of the gut microbiota and severely reduces antibody response to influenza vaccination. This impairment of humoral immunity correlated with significant impairments in cellular compartments that are crucial to the humoral immune response. ABX treatment resulted in reduced frequency and activation of a number of myeloid cell subsets in the dLN, both

prior to and post-vaccination. In addition, $\mathrm{T}_{\mathrm{FH}}$ cell number were significantly reduced in the $\mathrm{dLN}$ following vaccination and this was concurrent with reduced frequencies of CS GC B cells and PCs.

As differentiation and function of $\mathrm{T}_{\mathrm{FH}}$ and $\mathrm{B}$ cells are interdependent, these findings do not reveal if the defects induced by ABX treatment are intrinsic to $\mathrm{T}$ or B cells. Further investigation as to the involvement of microbial signals in the development of $\mathrm{T}$ and $\mathrm{B}$ cell responses to TIV vaccination are required to determine this. Additionally, it is clear that $A B X$ treatment has significant effects on myeloid cells. Given their role in antigen transport and presentation to both T and B cell within the dLN, reduced number and activatory potential of myeloid cells following antibiotic treatment likely contributes to defects in these cellular responses, ultimately leading to poor antibody responses to vaccination.

This data suggests that the gut microbiota may be acting as an adjuvant in the immune response to TIV vaccination and presents the potential for beneficial modulation of gut microbial communities to enhance vaccine responsiveness. 
4 The influence of functional foods on immune responses to influenza vaccination 


\subsection{Introduction}

Recent alterations to human lifestyles have had significant effects on the structure and function of our gut microbiota and abnormal microbiome structures (i.e. dysbiosis) are associated with the steep rise in prevalence of many immunological diseases, such as inflammatory bowel diseases, diabetes, asthma and susceptibility to infection ${ }^{235}$. There is mounting evidence that dysbiosis has causative role in these diseases ${ }^{185}$, as was shown in the previous chapter altering the microbiota with $\mathrm{ABX}$ has a detrimental impact on a number of immune cell populations at a systemic site and reduces the ability to mount protective antibody responses against influenza vaccination. Therefore, it is key that therapies are developed for the beneficial modulation of gut microbial communities to promote specific health outcomes. Diet has rapid and profound effects on microbiota community structure and function ${ }^{190,192}$, and presents an exciting opportunity to improve health outcomes for dysbiosis-related disease in a safe and affordable manner.

In conjunction with the High-Value Nutrition Science Challenge, this thesis seeks to validate the beneficial effects of New Zealand food products to build immune defence against influenza. If successful, this study will lead to clinical trials that will investigate the benefit of high-value nutritional products in improving host-microbiota interactions and enhancing immune responses following vaccination. Four nutritional interventions were selected to be trialed in this thesis for their ability to influence the immune response to influenza vaccination in a pre-clinical mouse model. Candidates selected were the probiotic, Bifidobacterium animalis and potential prebiotics, Sungold kiwifruit, propolis with $\gamma$-cyclodextrin formulation and fibre-modified diets. Kiwifruit consumption has been linked to modulation of immune function, shown to 
boosts baseline and vaccine-specific serum antibody responses in mice ${ }^{236-238}$, and there is some evidence of protection against upper respiratory tract infection in humans ${ }^{239}$. Kiwifruit is also rich in MACs, therefore is a purported prebiotic ${ }^{240}$. B. animalis is a probiotic bacterium and human intake has been shown to alter gut microbial communities, increasing butyrogenic bacteria and reducing pathobionts ${ }^{241}$, as well as significantly enhancing antibody production to influenza vaccination in healthy adults ${ }^{205}$. Propolis is a bee product and a potential prebiotic, it is rich in polyphenolic compounds ${ }^{242}$, many of which are substrates for bacterial fermentation to immunomodulatory compounds ${ }^{184,243}$ and propolis administration to rats has been shown to alter composition of the gut microbiota ${ }^{244}$. Increased dietary fibre intake has also been shown to enhance production of $\operatorname{Ig} G$ both at steady-state and following bacterial infection by fueling the production of microbiota-produced SCFAs ${ }^{175}$. Therefore, these foods have potential to alter the gut microbiota and improve influenza vaccine responses.

In the feeding experiments, mice are maintained on AIN93 rodent diet or this as the base for all dietary modifications. This was used for nutritional intervention studies because AIN93 is made from refined macronutrient sources and nutrient levels are more closely controlled compared to the standard cereal-based meatfree mouse diet. It also is lower in fibre and higher in fat, closer to the 'western' diet of the population we are modelling ${ }^{191}$.

In this chapter, mouse models of both human parenteral and mucosal vaccines are established. As discussed previously, the human parenteral influenza vaccination, TIV, induces a IgG antibody response, dominated by the IgG1 isotype, in the serum of recipients without inducing respiratory tract $\operatorname{Ig} \mathrm{A}^{107,108}$. FluMist, the intranasal live-attenuated influenza vaccine, induces a strong IgA antibody response in the respiratory tract, as well as serum $\operatorname{Ig}^{110-112}$. However, 
neither vaccine induces complete protection against infection, with many individuals eliciting suboptimal antibody responses ${ }^{115}$. In this section, as well as quantifying antibody production to vaccination, we wanted to assess protection against influenza infection following administration of functional foods. This secondary measure will ensure that if functional foods improve protection against infection, but do not increase antibody titres, alterations to other immune parameters can be detected. Mice are resistant to human influenza strains ${ }^{245}$ and we do not have the laboratory facilities to challenge mice with human strains present in the human TIV vaccine. Therefore, it was decided that HKx31 influenza virus, a mouse-adapted influenza A strain, would be used to manufacture a murine influenza vaccine. Transient weight loss and viral load in the lung will be measured following HKx31 infection as a measure of viral control $^{246}$.

\subsection{Aims}

For the murine influenza vaccine models to fulfil the requirements as a model for human TIV vaccination, it must induce the appropriate isotype of virus-specific antibody response, serum IgG by both the parenteral and mucosal vaccination route and respiratory tract $\operatorname{IgA}$ by mucosal vaccination. The virus-specific antibodies must be easily detectable by virus-specific ELISA. Antibody responses must also be suboptimal, below the maximal response in order for the response to be able to be improved through nutritional intervention. 
Specific aims:

1) To establish mouse models of parenteral and mucosal influenza vaccination that model low-responding human populations, inducing antibody production and protection against subsequent infection that are suboptimal.

2) To assess the capacity of oral administered nutritional interventions with microbiota-altering potential to improve suboptimal immune responses in the influenza vaccination model. 


\subsection{Results}

\subsubsection{Formalin-inactivated influenza A vaccine does not induce strong antibody responses when administered SC or IN}

To establish vaccination models, a whole inactivated virus vaccine was produced. HKx31 influenza A virus was killed by formalin inactivation according to methods described by Jonges and colleagues, who demonstrated complete viral inactivation ${ }^{213}$. Mice were administered increasing doses of the vaccine or PBS as a control, SC or IN once a week for three weeks. Multiple doses were administered in order to ensure induction of robust antibody responses according to previous investigations ${ }^{247,248}$. This also better models human vaccination, as pre-existing immunity to vaccine strains is common, due to previous vaccination or infection ${ }^{249}$. Mice were sacrificed one week following the final vaccination and serum, BAL and nasal lavage were collected to assess serum HKx31-specific total IgG and IgG1 antibody and HKx31-specific IgA antibody in the BAL and nasal lavage by ELISA assay (Figure 4.1A).

We have observed that 1 haemagglutination unit (HAU) - a unit of viral measurement - of live virus administered IN to naïve mice induces approximately 10\% weight loss three days following infection (Section 4.3.3). Administration of inactivated virus did not induce weight-loss compared to PBS administered mice, regardless of the dose or route of administration (Figure 4.1B and C). This further confirms complete viral killing by this method. As was expected, SC vaccination did not elicit detectable levels of virus-specific IgA in the nasal lavage (Figure 4.1D) or BAL (Figure 4.1E). Mice vaccinated SC at any viral dose did not elicit high levels of virus-specific total IgG (Figure 4.1F) or IgG1 (Figure 4.1G) antibody. At doses of 10 or $100 \mathrm{HAU}$, vaccines elicited detectable virus-specific IgG, however these did not reach statistically significant 
levels (Figure 4.1F). Low titres of IgA were detected in the nasal lavage (Figure $4.1 \mathrm{H}$ ) and BAL (Figure 4.1I) of some mice IN vaccinated at the highest dose of 50 HAU. However, no virus-specific total IgG (Figure 4.1J) or IgG1 (Figure 4.1K) were detected in the serum at any dose.

This data demonstrates that neither vaccine route elicited consistent virus-specific IgG antibody in the serum or IgA in the respiratory tract detectable by ELISA at the doses tested. Therefore, SC nor IN administration of the formalin-inactivated influenza vaccine at the doses tested will not serve as effective models of either the parenteral or mucosal human influenza vaccines. 
(A)

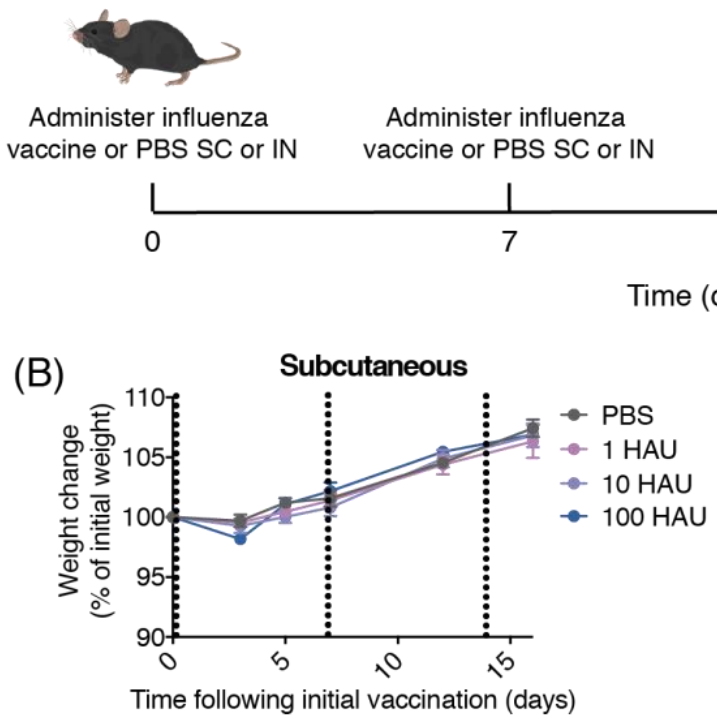
$\begin{array}{cc}\text { Administer influenza } & \text { Assess virus-specific } \\ \text { vaccine or PBS SC or IN } & \text { antibody production }\end{array}$

ime following initial vaccination (days)

Subcutaneous

(D)

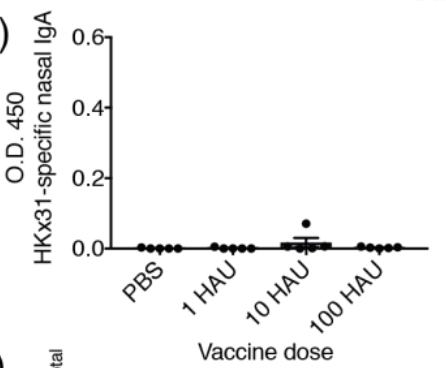

(F)

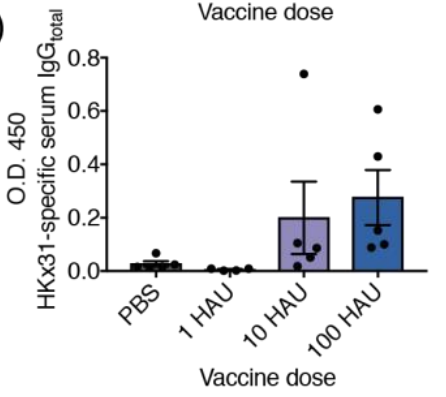

(C)

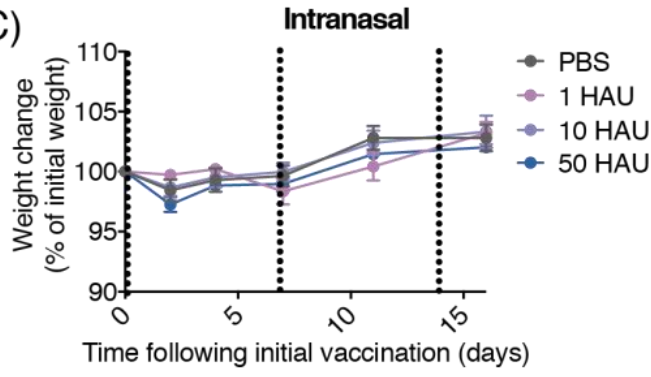

(E)

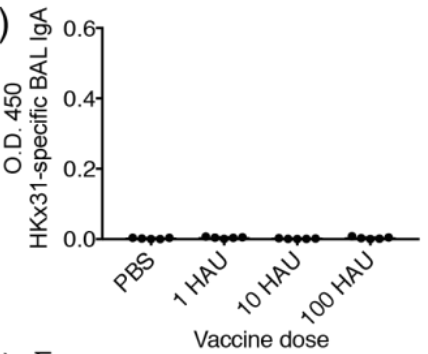

(G)

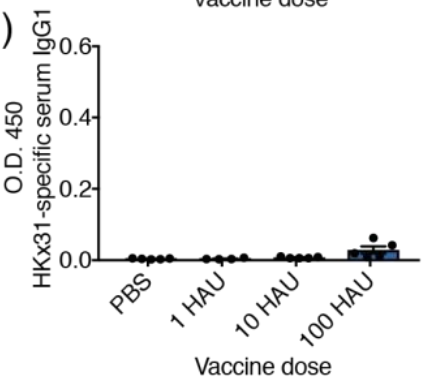

Intranasal

(H)

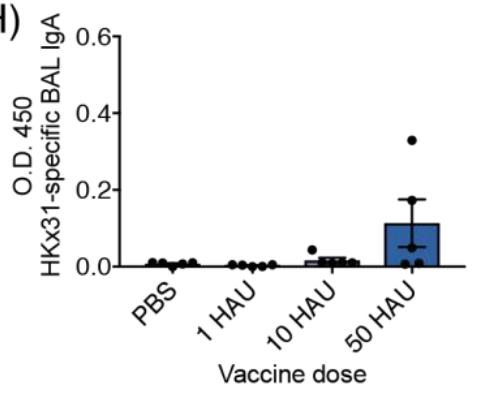

(J)

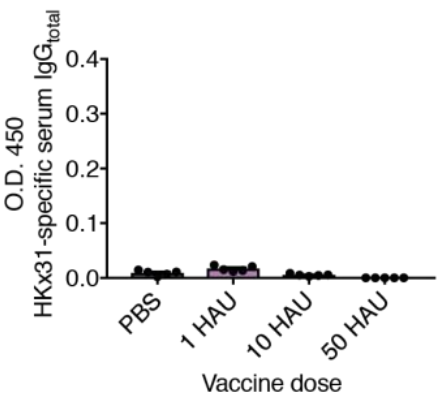

(I)

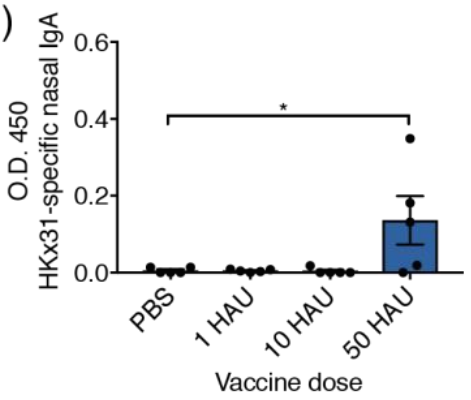

(K)

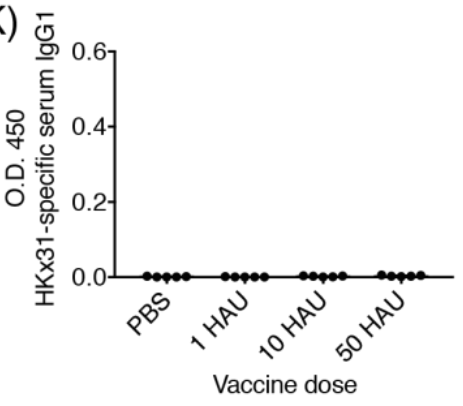


Figure 4.1: Formalin-inactivated influenza $A$ vaccine does not induce sufficient antibody production when administered SC or IN. (A) Experimental outline for administration of formalin-inactivated HKx31 vaccine. C57BL/6 mice were administered either $200 \mu \mathrm{L}$ SC or $50 \mu \mathrm{L}$ IN of vaccine or PBS once a week for three weeks. Seven days following the final vaccination, mice were culled and serum, nasal lavage and BAL were collected for measurement of HKx31-specific antibodies by ELISA. Weight change from initial weight of mice vaccinated with PBS or increasing doses of vaccine via (B) SC or (C) IN route. Horizontal dotted lines indicate the day of vaccination. HKx31-specific IgA in the (D) nasal lavage and (E) BAL seven days following final SC vaccination. HKx31-specific serum (F) total IgG and (G) IgG1 seven days following final SC vaccination. HKx31-specific IgA in the (H) nasal lavage and (I) BAL seven days following final IN vaccination. HKx31-specific serum (J) total IgG or (K) IgG1 seven days following final IN vaccination. Data shown is $n=5$ per group. Statistical analysis of antibody titres was performed using One-Way ANOVA with Tukey's post-test showing mean \pm SEM. * $p \leq 0.05$. 


\subsubsection{UV-inactivated influenza A virus induces robust serum and mucosal antibody responses}

Formalin inactivation utilised in the previous chapter required a dialysis step that likely resulted in loss of viral material, which may account for low antibody titres. To address this, UV inactivation was chosen as an alternative method as it allows for collection of all inactivated viral material and is a faster process. HKx31 virus was UV-inactivated according to methods described by Zou and colleagues, who demonstrated complete viral killing ${ }^{214}$. Mice were administered increasing doses of UV-inactivated HKx31 influenza A vaccine either SC or IN, three times on days zero, seven and 14 of the experiment. Mice were sacrificed seven days later and serum, BAL and nasal lavage were collected. HKx31-specific total IgG and IgG1 antibody in the serum and HKx31-specific IgA antibody in the BAL and nasal lavage were assessed by ELISA assay (Figure $4.2 \mathrm{~A})$.

Administration of inactivated virus did not induce weight-loss compared to PBS controls when administered SC (Figure 4.2B) or IN (Figure 4.2C), again confirming complete viral killing by UV inactivation. As expected, SC vaccination did not elicit detectable virus-specific IgA in the nasal lavage (Figure 4.2D) or BAL (Figure 4.2E). Robust serum total IgG (Figure 4.2F) and IgG1 isotype (Figure $4.2 \mathrm{G}$ ) antibodies were induced by SC vaccination at both doses tested, with lower levels of antibodies at the $200 \mathrm{HAU}$ vaccine dose compared to $500 \mathrm{HAU}$, although these did not reach statistical significance. IN vaccination elicited strong virus-specific IgA antibody production in the nasal lavage (Figure 4.2H) and BAL (Figure 4.2I) at the doses tested. Virus-specific BAL IgA and serum IgG1 were significantly lower at the $200 \mathrm{HAU}$ dose than $500 \mathrm{HAU}$, while nasal IgA and serum total IgG trended to lower levels. 
This data demonstrates that the UV-inactivated 200 HAU vaccine dose, administered SC or IN, can serve as appropriate models for suboptimal parenteral and mucosal vaccination, respectively. Vaccinations induced appropriate antibody responses that were easily detectable by ELISA assay. Serum IgG, including of the IgG1 isotype, was elicited by both vaccine routes, while only IN vaccination induced respiratory tract IgA. Importantly, the chosen dosage does not induce a maximal antibody response, and therefore has the potential to be improved with nutritional interventions. 
(A)
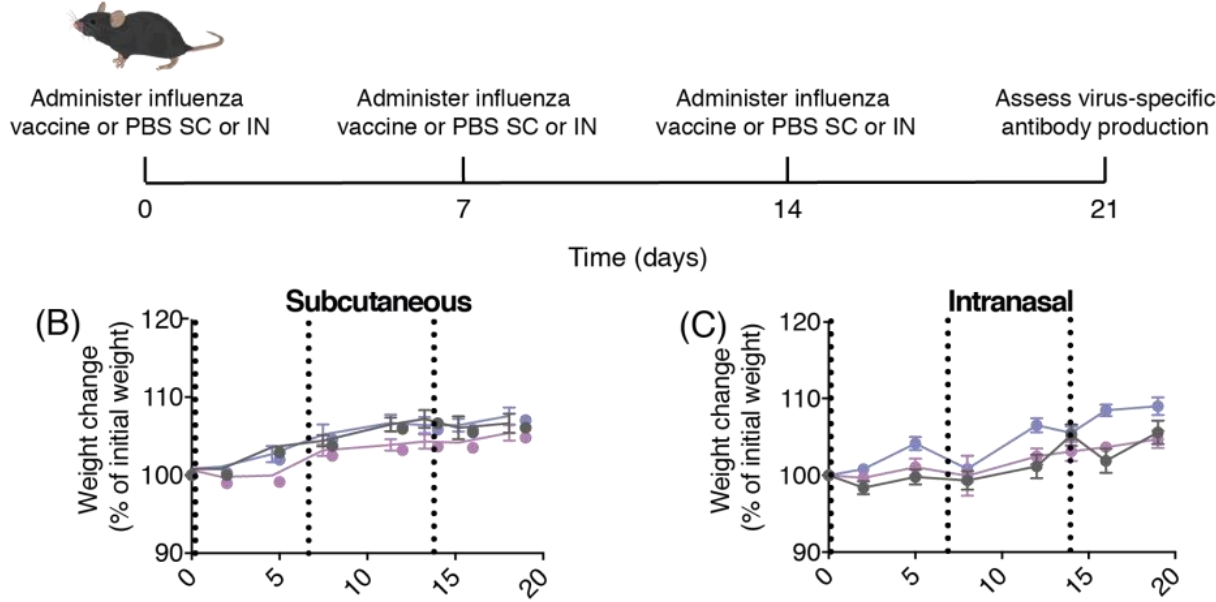

Time (days)

Time following inital vaccination (days)

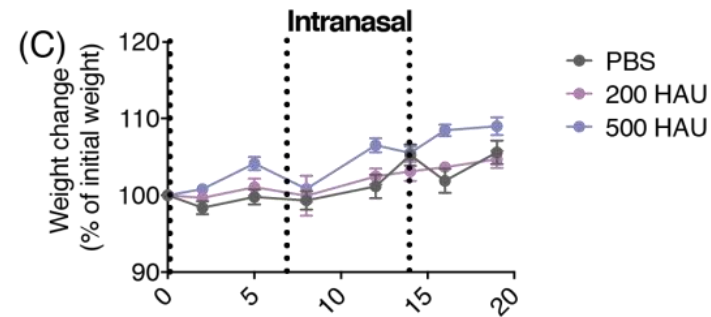

Subcutaneous

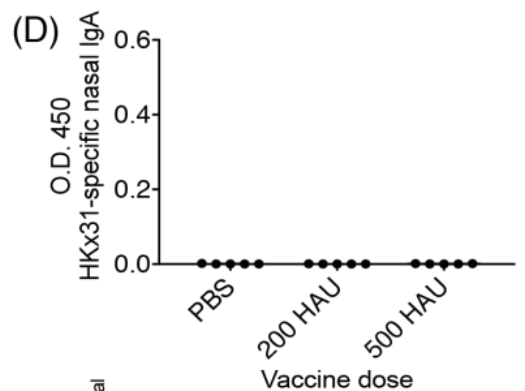

Time following initial vaccination (days)

(F)

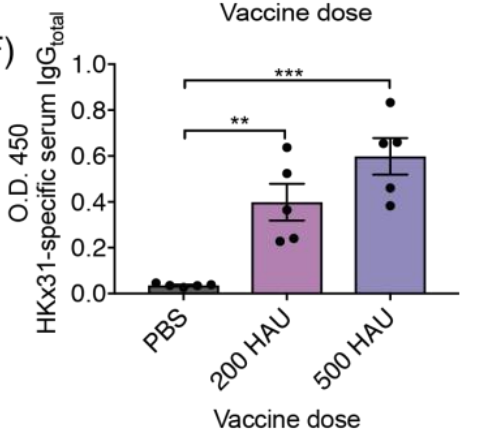

(E)

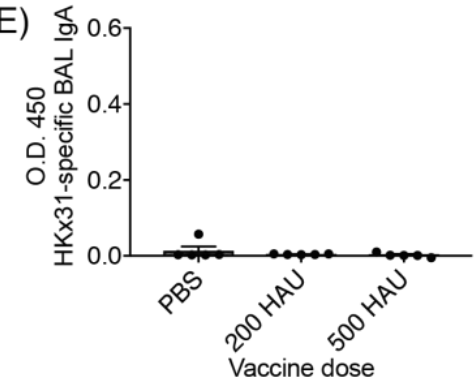

(G)

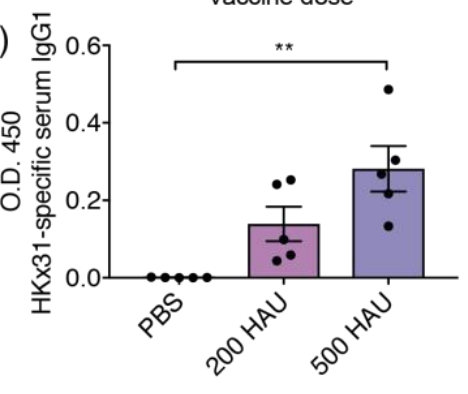

Vaccine dose

Intranasal
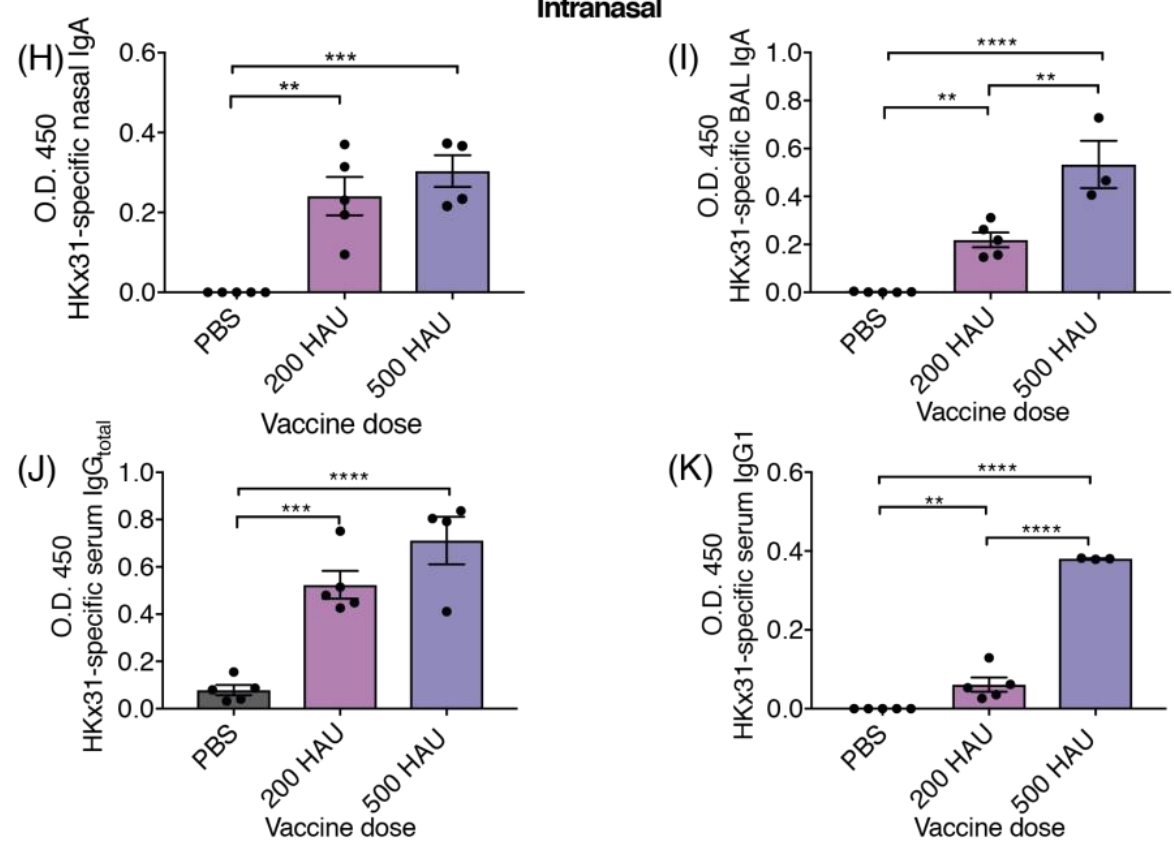
Figure 4.2: UV-inactivated influenza A virus induces strong serum and mucosal antibody responses. (A) Experimental outline for administration of UV-inactivated HKx31 vaccine. C57BL/6 mice were administered either $200 \mu \mathrm{L}$ SC or $50 \mu \mathrm{L}$ IN of vaccine or PBS once a week for three weeks. Seven days following the final vaccination, mice were culled and serum, nasal lavage and BAL were collected for measurement of HKx31-specific antibodies by ELISA. Weight change from initial weight throughout experiment for mice vaccinated via (B) SC or (C) IN route. Horizontal dotted lines indicate day of vaccination. HKx31-specific IgA in the (D) nasal lavage and (E) BAL seven days following final SC vaccination. HKx31-specific serum (F) total IgG and (G) IgG1 seven days following final SC vaccination. HKx31-specific IgA in the $(\mathbf{H})$ nasal lavage and (I) BAL seven days following final IN vaccination. HKx31-specific serum (J) total IgG or (K) IgG1 seven days following final IN vaccination. Data shown is $n=3-5$ per group. Statistical analysis of antibody titres was performed using One-Way ANOVA with Tukey's post-test. All data is displayed as mean \pm SEM. ${ }^{* *} \mathrm{p} \leq 0.01,{ }^{* * *} \mathrm{p} \leq 0.001,{ }^{* * * *} \mathrm{p} \leq 0.0001$. 


\subsubsection{Administration of inactivated influenza vaccines protect against infection}

Next, the protection against subsequent infection with live HKx31 influenza virus induced by each vaccine was determined. To assess this, mice were administered increasing doses of UV-inactivated $\mathrm{HK} \times 31$ influenza A vaccine either SC or IN, once a week for three weeks. Seven days following the final vaccination, mice were infected with $1 \mathrm{HAU}$ of live $\mathrm{HK} \times 31$ influenza virus. The ability to control infection was assessed by infection-induced weight loss and the quantity of viral RNA in the lung relative to that of mice administered PBS alone at three days post-infection (Figure 4.3A).

Although not statistically significant, SC vaccination of 200 HAU protected three of the four mice from infection-induced weight loss. In contrast, those administered lower doses lost approximately $10 \%$ of their body weight, comparable to weight loss observed in PBS control mice (Figure 4.3B). Despite no significant protection from weight loss, significantly less viral RNA was measured in the lungs of vaccinated mice at all doses tested compared to PBS control mice, however viral RNA was readily detectable by qPCR (Figure 4.3C), indicating incomplete protection. IN vaccination induced a far more potent protective effect at the same dose, with vaccine doses of 50 and 200 HAU inducing significant protection from the infection-induced weight loss observed in the PBS group, only one mouse exhibited weight loss at the lowest dose of 5 HAU (Figure 4.3D). Strikingly, IN vaccination resulted in viral RNA levels that were undetectable by qPCR at three days post-infection, except for one mouse administered the lowest dose (Figure 4.3E), the same individual that lost $10 \%$ of body weight following infection (Figure 4.3D). 
This experiment demonstrates that both SC and IN administration of UV-inactivated influenza vaccine at $200 \mathrm{HAU}$ elicits robust protection against subsequent viral infection. SC administration of 200 HAU elicited both suboptimal infection-induced weight loss and protection from infection. However, a much stronger protective response was elicited by IN vaccination, where $200 \mathrm{HAU}$ dosage elicited complete protection against weight loss and no detectable viral RNA in the lung. 
(A)

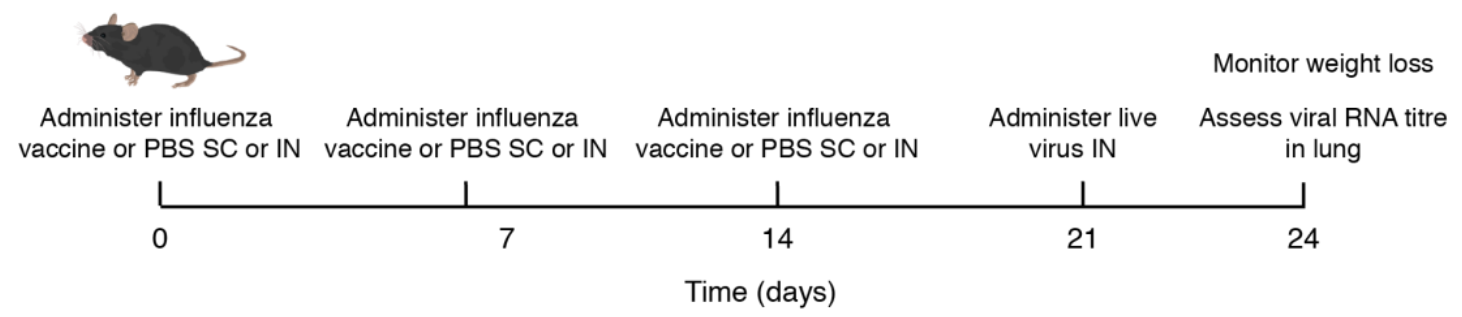

Subcutaneous

(B)

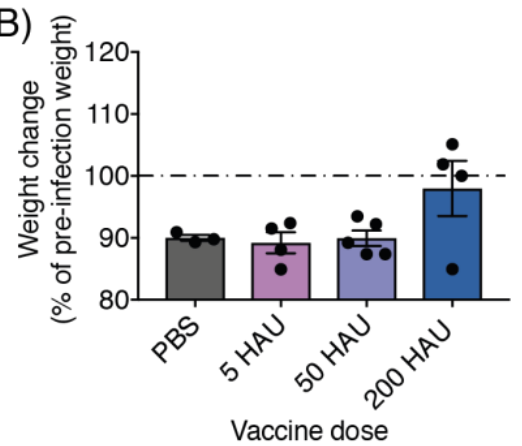

Intranasal

(D)

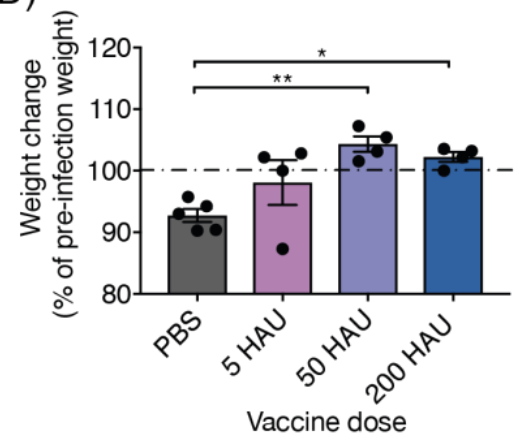

(C) $\sum_{\square}$

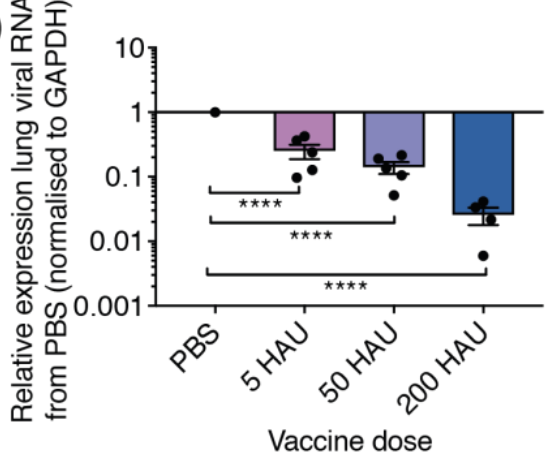

(E)

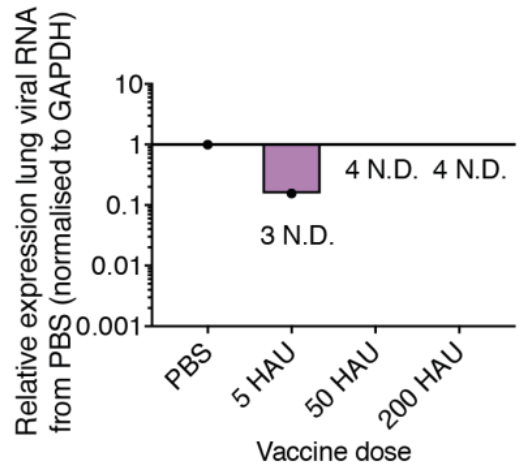

Figure 4.3: Parenteral and mucosal influenza vaccines induce protection against infection. (A) Experimental outline for administration of UV-inactivated HKx31 vaccine followed by viral challenge. C57BL/6 mice were administered either $200 \mu \mathrm{L}$ SC or $50 \mu \mathrm{L}$ IN of vaccine or PBS once a week for three weeks. Seven days following the final vaccination, mice infected with $1 \mathrm{HAU}$ of live HKx31 virus in $50 \mu \mathrm{L}$ IN. Three days following infection, mice were sacrificed and lungs were collected for quantitation of viral RNA by qPCR. Weight change was monitored following infection. (B) Weight change three days following infection for mice vaccinated SC. (C) Expression of viral RNA in the right lobe of the lung at three days following infection of mice vaccinated SC. Viral RNA levels are normalised to GAPDH and calculated relative to PBS. (D) Weight change three days following infection for mice vaccinated IN. (E) Expression of viral RNA in the right lobe of the lung at three days following infection of mice vaccinated IN. Viral RNA levels are normalised to GAPDH and calculated relative to PBS. N.D.=non-detectable. Data shown is $n=3-5$ per group. Statistical analysis was performed using One-Way ANOVA with Tukey's post-test showing mean \pm SEM. ${ }^{*} \mathrm{p} \leq 0.05,{ }^{* * *} \mathrm{p} \leq 0.001,{ }^{* * * *} \mathrm{p} \leq 0.0001$. 


\subsubsection{Oral administration of kiwifruit does not improve antibody responses to influenza vaccination or protection from infection}

SC or IN administration of UV-inactivated HKx31 influenza vaccine at $200 \mathrm{HAU}$ dose induces robust antibody production and protection against infection, however, only SC administration elicits suboptimal responses. While IN administration at the same dose elicits suboptimal antibody responses, protection against infection is complete and no virus can be detected in the lung even at very low doses. Therefore, it would be impossible to determine an IN dose that would generate antibody readily detectable by ELISA assay, while still eliciting suboptimal response against infection. Furthermore, to assess mucosal antibody titres, mice must be sacrificed, therefore it is not possible to perform viral challenge simultaneously in the same mice. Based on this, it was decided to go forward only using SC administration of UV-inactivated virus at a $200 \mathrm{HAU}$ dose to model the human parenteral vaccination in subsequent nutritional intervention studies.

To investigate the efficacy of kiwifruit to improve immune responses to influenza vaccination, mice were administered kiwifruit or control solution intragastrically (IG) once a day, for five days each week, from two weeks prior to vaccination and throughout the rest of the experiment whilst being maintained on AIN93 rodent diet. Mice were administered 200 HAU of UV-inactivated HKx31 influenza A vaccine SC once a week for three weeks. Seven days following the final vaccination, serum was collected for analysis of virus-specific antibody and mice were infected with 1 HAU of live HKx31 influenza virus. The ability to control infection was assessed three days post-infection by weight loss and the quantity of viral RNA in the lung, relative to that of mice administered PBS and control feed (Figure 4.4A). 
Both influenza vaccine and PBS mice that received kiwifruit did not gain as much weight as their respective control groups (Figure 4.4B), and ate less food each week (Figure 4.4C). In response to influenza vaccination, mice fed kiwifruit had lower levels of virus-specific total IgG (Figure 4.4D) and IgG1 (Figure 4.4E) in the serum than control mice. Vaccination reduced viral RNA load in the lung three days following infection, however kiwifruit feeding did not improve viral control, with both the PBS and influenza vaccine groups exhibiting similar viral load between feeding regimes (Figure $4.4 \mathrm{~F}$ ). While vaccination protected mice against infection-induced weight loss, there was no difference in weight loss in the PBS groups, and influenza vaccinated mice receiving kiwifruit lost significantly more weight than control fed mice comparable to non-vaccinated mice (Figure $4.4 \mathrm{G}$ ).

Collectively, these data demonstrate that influenza vaccinated mice fed with kiwifruit elicited lower levels of virus-specific antibody and, while they did not have significantly more viral RNA in the lung following infection, they were more susceptible to infection-induced weight loss. This coincided with reduced food intake in kiwifruit fed groups and subsequent failure to gain as much weight as control mice. 
(A)

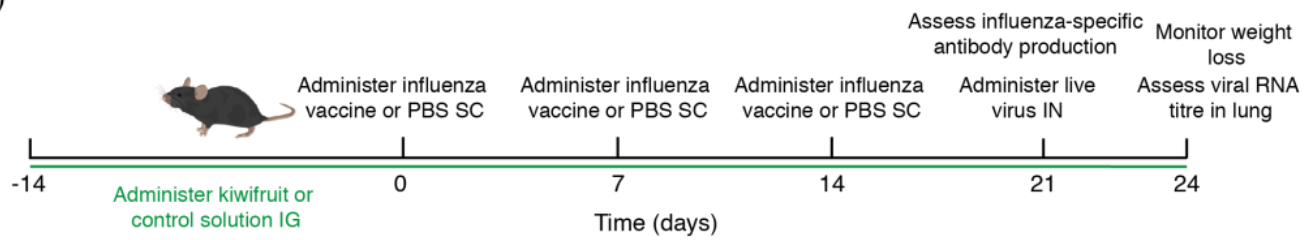

(B)


(C)

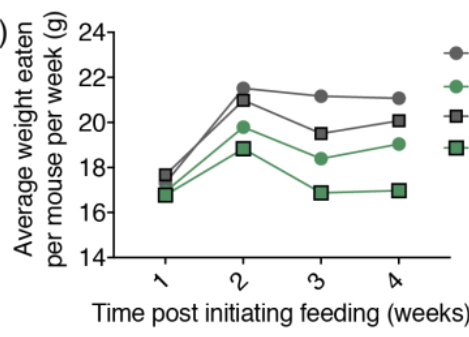

(E)

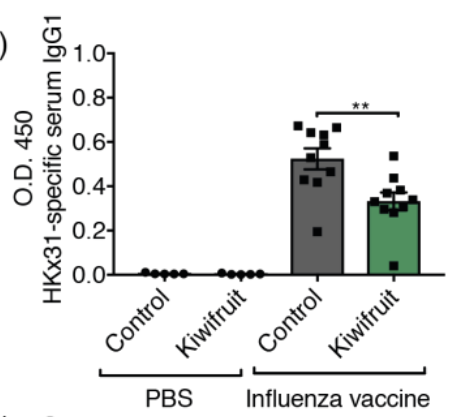

(G)

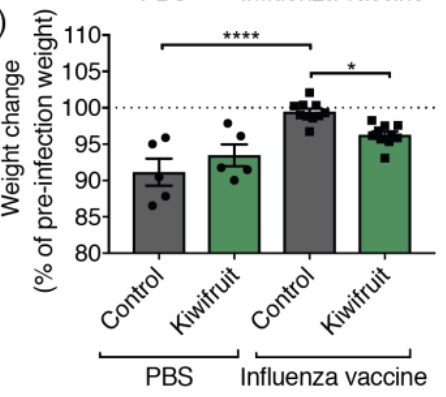

Figure 4.4: Administration of kiwifruit is detrimental to antibody responses to influenza vaccination and protection from infection. (A) Experimental outline for administration of kiwifruit and UV-inactivated HKx31 vaccine followed by viral challenge. C57BL/6 mice were vaccinated SC with $200 \mathrm{HAU}$ of UV-inactivated influenza virus in $200 \mu \mathrm{L}$ or PBS once a week for three weeks. Mice were administered 300 to $500 \mu \mathrm{L}$ of kiwifruit IG five days per week from 14 days prior to initial vaccination and for the entirety of the experiment whilst being maintained on AIN93 rodent diet. Seven days following the final vaccination, mice were infected with $1 \mathrm{HAU}$ of live HKx31 virus in $50 \mu \mathrm{L}$ IN. Three days following infection, weight loss was measured, mice were sacrificed and lungs were collected for quantitation of viral RNA by qPCR. (B) Weight change from initial weight of mice throughout the experiment. (C) Average weight of rodent diet eaten per mouse per week throughout experiment. HKx31-specific serum (D) total IgG and (E) IgG1 seven days following final vaccination. (F) Expression of viral RNA in the right lobe of the lung at three days following infection. Viral RNA levels are normalised to GAPDH and calculated relative to PBS. (G) Weight change three days following infection. Data shown is $\mathrm{n}=5-10$ per group. Statistical analysis was performed using One-Way ANOVA with Tukey's post-test showing mean \pm SEM. ${ }^{*} \mathrm{p} \leq 0.05,{ }^{* *} \mathrm{p} \leq 0.01,{ }^{* * * *} \mathrm{p} \leq 0.0001$. 


\subsubsection{Oral administration of probiotic $B$. animalis does not enhance antibody responses to influenza vaccination or protect from infection}

Next, the impact of oral administration of the probiotic bacteria, B. animalis, to improve immune responses to influenza vaccination and subsequent infection was assessed. To test this, mice were administered B. animalis or control solution IG once a day, five days each week, from two weeks prior to vaccination and throughout the rest of the experiment whilst being maintained on AIN93 rodent diet. Mice were administered $200 \mathrm{HAU}$ of UV-inactivated HKx31 influenza A vaccine SC once a week for three weeks. Seven days following the final vaccination, serum was collected for analysis of virus-specific antibody then mice were infected with $1 \mathrm{HAU}$ live $\mathrm{HK} \times 31$ influenza virus. The ability to control infection three days post-infection was assessed by infection-induced weight loss and the quantity of viral RNA in the lung, relative to that of mice administered PBS and control solution (Figure 4.5A).

Weight gain was similar between B. animalis and control fed groups (Figure 4.5B) and there were no significant differences in food intake between all groups (Figure 4.5C). Following influenza vaccination, B. animalis and control fed mice exhibited similar levels of virus-specific total IgG (Figure 4.5D) and IgG1 (Figure 4.5E). Following infection, B. animalis feeding did not improve control infection compared to those fed control solution, both in mice administered the influenza vaccine or PBS. Vaccination reduced viral load in the lung (Figure 4.5F) and protected mice against infection-induced weight loss (Figure 4.5G). B. animalis treated mice had comparable levels of viral RNA in the lung (Figure 4.5F) and exhibited similar weight change following infection (Figure 4.5G). 
These data demonstrate that oral administration of B. animalis does not improve antibody production to an inactivated influenza virus vaccine or control of subsequent infection. 
(A)

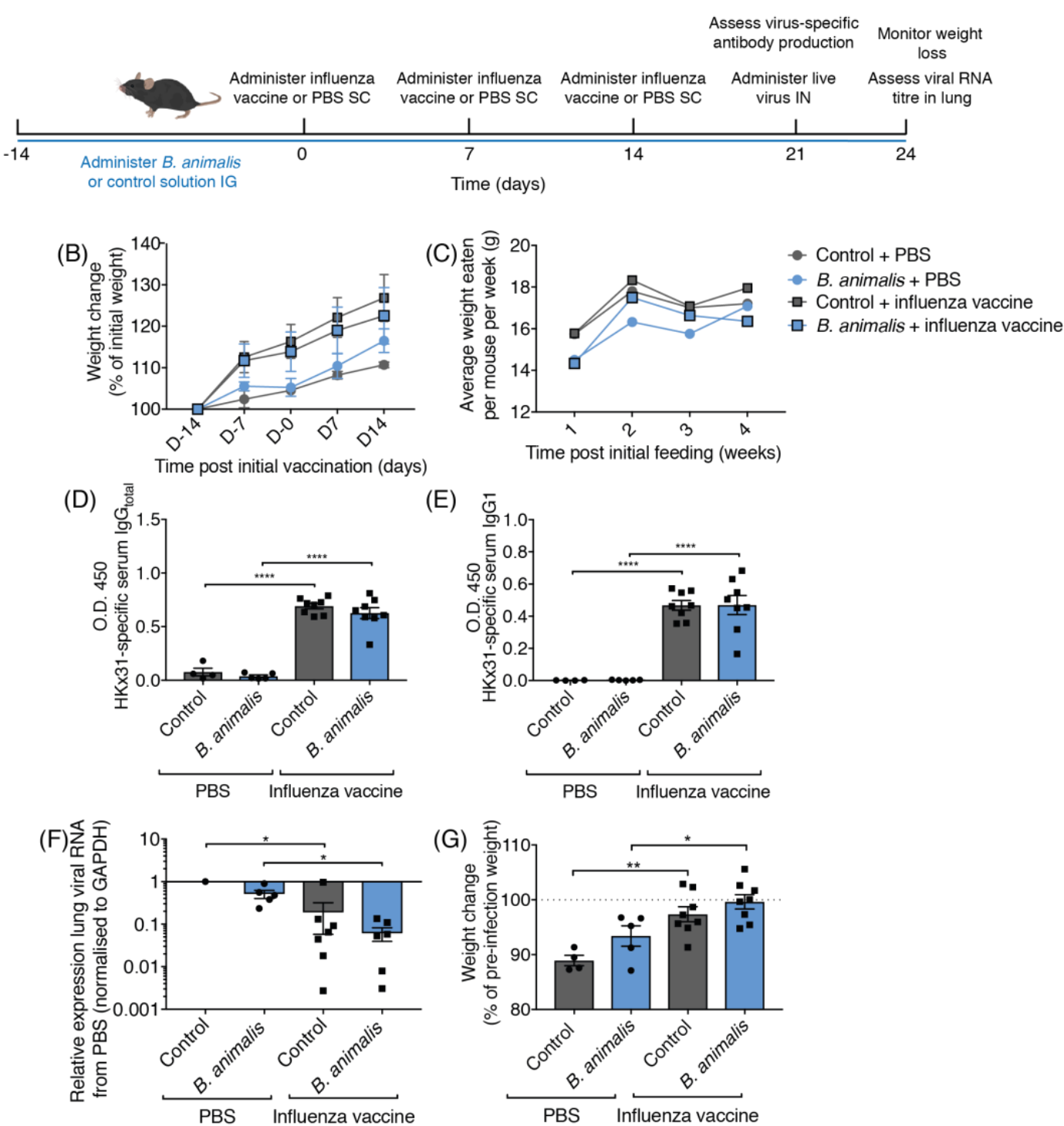

Figure 4.5: Oral administration of $B$. animalis elicits has no effect on vaccine-induced antibody production or protection against infection. (A) Experimental outline for administration of $B$. animalis and UV-inactivated $\mathrm{HK} \times 31$ vaccine followed by viral challenge. C57BL/6 mice were vaccinated SC with $200 \mathrm{HAU}$ of UV-inactivated influenza virus in $200 \mu \mathrm{L}$ or PBS once a week for three weeks. Mice were administered $1 \times 10^{9} \mathrm{CFU}$ of $B$. animalis in $200 \mu \mathrm{L}$ IG five days per week from 14 days prior to initial vaccination and for the entirety of the experiment whilst being maintained on AIN93 rodent diet. Seven days following the final vaccination, mice were infected with $1 \mathrm{HAU}$ of live HKx31 virus in $50 \mu \mathrm{L}$ IN. Three days following infection, weight loss was measured and mice were sacrificed and lungs were collected for quantitation of viral RNA by qPCR. (B) Weight change from initial weight of mice throughout the experiment. (C) Average weight of rodent diet eaten per mouse per week throughout experiment. HKx31-specific serum (D) total IgG and (E) IgG1 seven days following final vaccination. (F) Expression of viral RNA in the right lobe of the lung at three days following infection. Viral RNA levels are normalised to GAPDH and calculated relative to PBS. (G) Weight change three days following infection. Data shown is $n=5-10$ per group. Statistical analysis was performed using One-Way ANOVA with Tukey's post-test showing mean \pm SEM. ${ }^{*} \mathrm{p} \leq 0.05,{ }^{* *} \mathrm{p} \leq 0.01,{ }^{* * * *} \mathrm{p} \leq 0.0001$. 


\subsubsection{Oral administration of propolis formulation improves protection against infection without altering antibody responses}

Next, the effect of formulated propolis on immune responses to influenza vaccination and infection was investigated. To test this, mice were administered AIN93G rodent diet supplemented with $8 \%$ propolis formulated with cyclodextrin powder (propolis formulation) or AIN93G alone ad libitum from two weeks prior to vaccination and throughout the rest of the experiment. Mice were administered $200 \mathrm{HAU}$ of UV-inactivated HKx31 influenza A vaccine once a week for three weeks. Seven days following the final vaccination, serum was collected for analysis of virus-specific antibody then mice were infected with 1 HAU live HKx31 influenza virus. Infection-induced weight loss and the quantity of viral RNA in the lung, relative to that of mice administered PBS and control feed, was assessed at three days post-infection (Figure 4.6A).

Influenza vaccinated mice administered the propolis formulation diet tended to gain less weight than those fed the control diet (Figure 4.6B), however apart from the first week of the experiment, mice consumed a similar amount of diet (Figure 4.6C). Both IgG and IgG1 antibody titres were comparable between mice fed the propolis formulation and control diets (Figures 5.6D and 5.6E). Vaccination reduced viral load in the lung (Figure 4.6F), however did not elicit protection against infection-induced weight loss compared to PBS controls (Figure 4.6G). Despite no differences in virus-specific antibody, there was a reduction in viral load in influenza vaccinated mice consuming the propolis formulation compared to those fed the control diet, with half of the individuals exhibiting no detectable viral RNA in the lung three days post infection (Figure 4.6G). Mice consuming propolis formulation and administered PBS also tended to have reduced viral load in the lung (Figure 4.6F). However, weight loss following 
infection was comparable between propolis formulation and control groups (Figure 4.6G).

Collectively, this data suggests that oral administration of the propolis formulation may improve protective immunity against influenza infection following vaccination. However, this was not due to improving the production of virus-specific IgG antibodies elicited by the vaccination. 
(A)

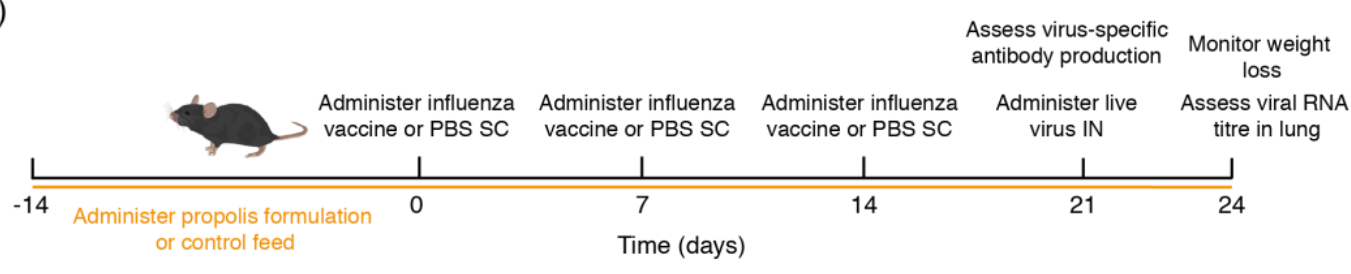

(B)

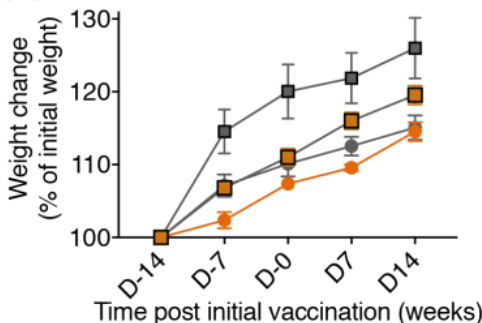

(D) 퓸

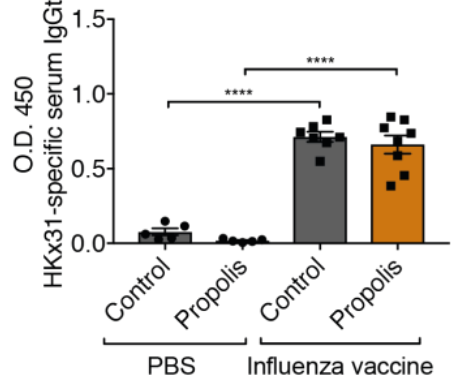

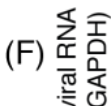

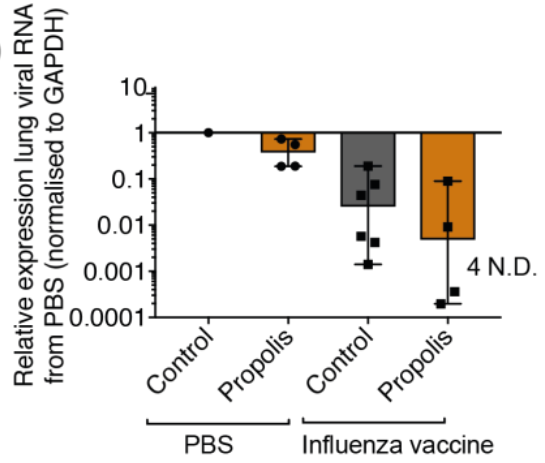

(C)

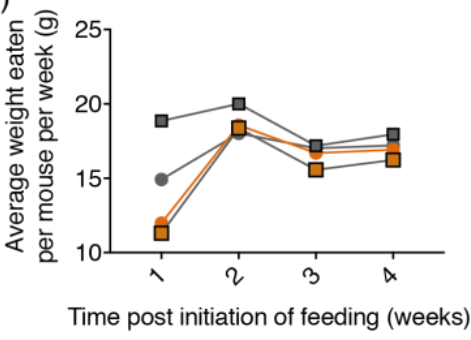

(E)

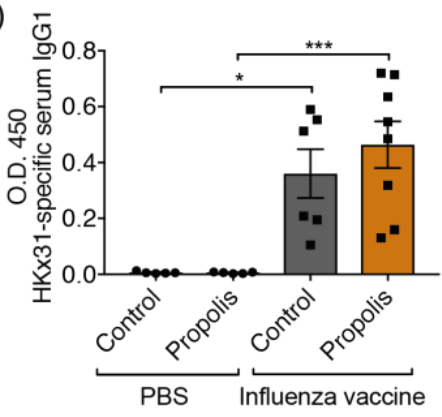

(G)

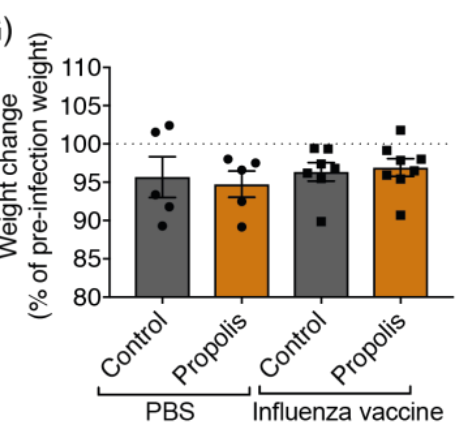

- Control + PBS

- Propolis + PBS

- Control + influenza vaccine

$\square$ Propolis + influenza vaccine 
Figure 4.6: Propolis formulation intake elicits some protection against influenza infection without influencing vaccine-induced antibody production. (A) Experimental outline for administration of propolis formulation and UV-inactivated $\mathrm{HK} \times 31$ vaccine followed by viral challenge. C57BL/6 mice were vaccinated SC with $200 \mathrm{HAU}$ of UV-inactivated influenza virus in $200 \mu \mathrm{L}$ or PBS once a week for three weeks. Mice were administered modified AIN93G rodent diet with $8 \%$ propolis cyclodextrin powder (propolis formulation) ad libitum from 14 days prior to initial vaccination and for the entirety of the experiment. Seven days following the final vaccination, mice were infected with $1 \mathrm{HAU}$ of live $\mathrm{HK} \times 31$ virus in $50 \mu \mathrm{L}$ IN. Three days following infection, weight loss was measured, mice were sacrificed and lungs were collected for quantification of viral RNA by qPCR. (B) Weight change from initial weight of mice throughout the experiment. (C) Average weight of rodent diet eaten per mouse per week throughout experiment. HKx31-specific serum (D) total IgG and (E) IgG1 seven days following final vaccination. (F) Expression of viral RNA in the right lobe of the lung at three days following infection. Viral RNA levels are normalised to GAPDH and calculated relative to PBS. N.D.=non-detectable. (G) Weight change three days following infection. Data shown is $n=5-10$ per group. Statistical analysis was performed using One-Way ANOVA with Tukey's post-test showing mean \pm SEM. ${ }^{*} \mathrm{p} \leq 0.05,{ }^{* * *} \mathrm{p} \leq 0.001,{ }^{* * * *} \mathrm{p} \leq 0.0001$. 


\subsubsection{Dietary fibre intake does not alter antibody responses to vaccination or prevent infection-induced weight loss}

Next, alterations to fibre content of the murine diet were assessed for the influence on immune responses to influenza vaccination and infection. To test this, mice were administered either zero-fibre, zero-starch rodent diet $(\mathrm{ZF})$ or a high-fibre equivalent diet formulated with $7.5 \%$ pectin and $7.5 \%$ inulin (HF) ad libitum from weaning (three weeks prior to vaccination) and throughout the rest of the experiment. Mice were administered $200 \mathrm{HAU}$ of UV-inactivated HKx31 influenza A vaccine once a week for three weeks. Seven days following the final vaccination, serum was collected for analysis of virus-specific antibody then mice were infected with $1 \mathrm{HAU}$ live HKx31 influenza virus. The ability to control infection was assessed by infection-induced weight loss and the quantity of viral RNA in the lung, relative to that of mice administered PBS and control feed, was assessed at three days post-infection (Figure 4.7A). Due to time constraints, the viral RNA load in the lung following infection could not be included in this thesis.

Weight gain throughout the experiment was comparable between mice fed ZF and HF diets (Figure 4.7B). Groups fed the ZF diet appeared to consume more food in the earlier weeks of the experiment, however as time went on food intake became equal between feeding groups (Figure 4.7C). Following vaccination, both ZF and HF fed mice mounted strong vaccine-specific IgG and IgG1 antibody responses, however fibre content of the diet had no impact on antibody responses to vaccination (Figure 4.7D and E). Moreover, following influenza infection, there was no difference in infection-induced weight loss between feeding groups (Figure 4.7F). 
These data demonstrate that content of dietary fibre, specifically pectin and inulin, has no impact on antibody responses to influenza vaccination or protection against weight loss following infection. 
(A)

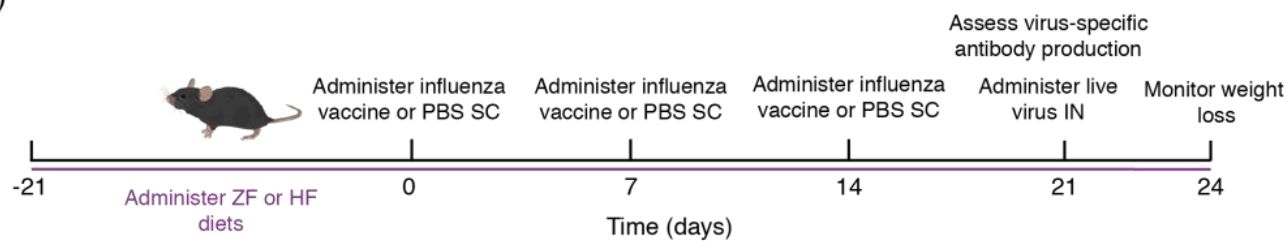

(B)

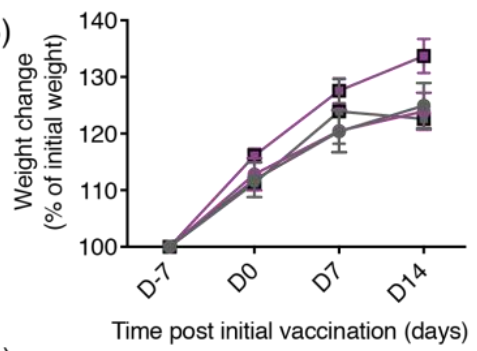

(D) तु

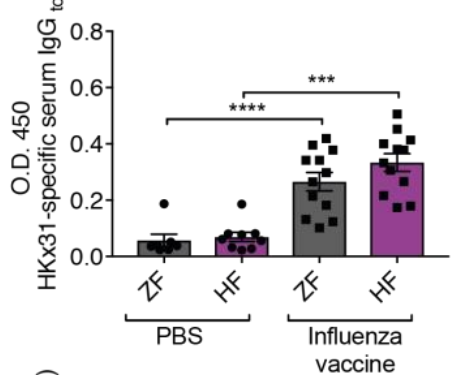

(F)

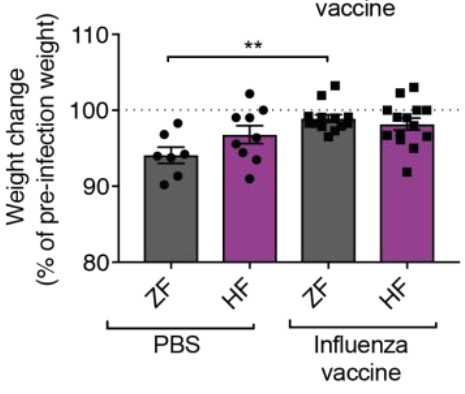

(C)

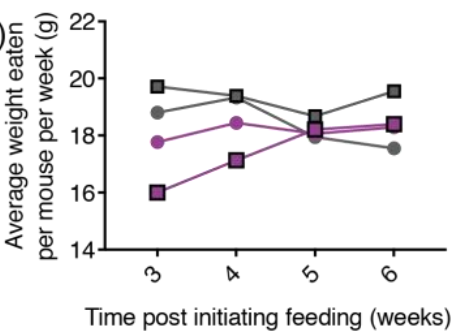

(E)

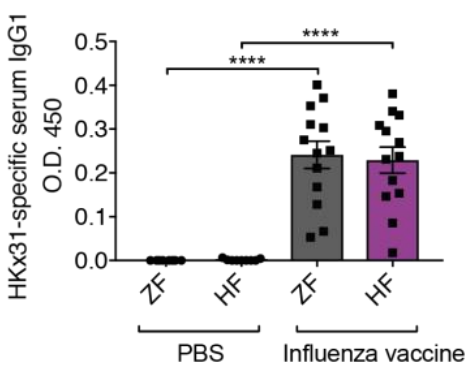

- ZF+ PBS

$-\mathrm{HF}+\mathrm{PBS}$

- ZF + influenza vaccine

$\square \mathrm{HF}+$ influenza vaccine

Figure 4.7: Fibre intake does not influence antibody production to vaccination or protection against infection-induced weight loss. (A) Experimental outline for administration of fibremodified diets and UV-inactivated HKx31 vaccine followed by viral challenge. C57BL/6 mice were vaccinated SC with $200 \mathrm{HAU}$ of UV-inactivated influenza virus in $200 \mu \mathrm{L}$ or PBS once a week for three weeks. Mice were administered zero-fibre, zero-starch SF15-086 rodent (ZF) diet or modified SF15-086 with 7.5\% pectin, 7.5\% inulin (HF) diet ad libitum from weaning 21 days prior to initial vaccination and for the entirety of the experiment. Seven days following the final vaccination, mice were infected with $1 \mathrm{HAU}$ of live $\mathrm{HKx} 31$ virus in $50 \mu \mathrm{L}$ IN. Three days following infection, weight loss was measured, mice were sacrificed. (B) Weight change from initial weight of mice throughout the experiment. (C) Average weight of rodent diet eaten per mouse per week throughout experiment. HKx31-specific serum (D) total IgG and (E) IgG1 seven days following final vaccination. (F) Weight change three days following infection. Data shown is $n=5-10$ per group. Statistical analysis was performed using One-Way ANOVA with Tukey's post-test showing mean \pm SEM. ${ }^{* *} \mathrm{p} \leq 0.01,{ }^{* * *} \mathrm{p} \leq 0.001,{ }^{* * * *} \mathrm{p} \leq 0.0001$. 


\subsection{Discussion}

The aim of this chapter was to establish mouse models of parenteral and mucosal influenza vaccination capable of inducing suboptimal antibody production and protection against subsequent infection, in order to model low-responding human populations. This model was used to determine if administration of potential microbiota-altering nutritional interventions improved immune responses to the vaccine. UV-inactivated live influenza virus induced potent antibody production and protection against infection when administered via parenteral and mucosal routes. However, mucosal vaccination gave full protection against infection, even at very low doses, meaning there was no opportunity to improve protection with nutritional interventions. Therefore, only the parenteral vaccine was taken forward for further studies. Administration of kiwifruit, $B$. animalis or a high fibre diet induced no discernible improvements in immune responses to vaccination or subsequent infection based on the parameters measured. In contrast, while there was no difference in vaccine-induced antibody production and weight change following infection upon administration of the propolis formulation, mice receiving this nutritional intervention tended to be better protected against infection, with a trend towards lower viral RNA loads in the lung.

When establishing the inactivated viral vaccine models, mice administered formalin-inactivated virus produced negligible antibody in response to vaccination. It has been observed that formalin inactivation may modify antigenic structures of vaccine proteins ${ }^{250}$, a potential contribution to observed low antibody titres. However, the dosages tested were also lower than those used for the UV-inactivated vaccine and this likely contributed to poor antibody 
responses to the vaccine and there was insufficient time to perform another dose titration.

It should be noted that the virus-specific total IgG ELISA may not be an optimal assay. Naïve control mice are expected to have no virus-specific IgG in their serum, yet for these mice O.D. values are consistently above zero for the IgG ELISA, yet not for the IgG1 isotype ELISA. The difference between these assays is the secondary antibody, the IgG secondary antibody is likely binding non-specifically to other components of the serum bound to the plate. To rectify this issue in further investigations, O.D. values could be normalised to untreated samples. However, the same trends between groups would be expected. Alternatively, the ELISA could be optimised with different blocking and sample-dilution reagents to minimise background signal.

Previous studies have shown that kiwifruit administration was able to improve antibody responses to peptide vaccination in mice ${ }^{236}$. Surprisingly, in this model, mice administered kiwifruit exhibited a significant reduction in virus-specific antibodies and lost more weight following infection than controls, while influenza RNA load was unchanged. It should be noted that those fed kiwifruit also tended to eat less of their regular food and gain less weight throughout the experiment, likely because they were administered a large volume of kiwifruit daily. Decreased body weight and possible adverse effects from kiwifruit administration may have led to lower antibody responses. Further investigation with a lower dosage is required to determine the efficacy of kiwifruit in altering antibody responses to influenza vaccination.

Contrary to improvements in vaccine-induced antibody titres observed in a large human cohort administered B. animalis ${ }^{205}$, neither vaccine-specific antibody production nor protection against infection were improved in mice administered 
B. animalis. Probiotics have been shown to successfully engraft in the microbiota of only certain individuals ${ }^{251}$, suggesting that the microbiota of these mice may not have been permissive to colonisation by this strain. This could also be reflective of inherent differences in the physiology and microbiota between humans and mice ${ }^{252}$.

It has been previously shown that consumption of a diet high in inulin and pectin fibre leads to significantly increased levels of circulating SCFAs compared to mice fed a zero-fibre diet and this boosted IgG production at baseline and following bacterial infection ${ }^{175}$. Despite utilising the same dietary regime as this published investigation, no discernible difference in antibody production or protection against infection-induced weight loss between mice fed high or zero fibre diets was observed. However, the difference between mounting an antibody response to bacterial infection compared to influenza vaccination may account for this discrepancy. Additionally, differences in baseline microbiota composition between these investigations could have played a role, as it has been observed that the gut microbiota of mice significantly differs between research institutes, which can significantly impact outcomes ${ }^{253}$. Also, contrary to the investigation by Kim and colleagues, it has been observed that SCFAs can inhibit B cell CSR, SHM and PC differentiation and SCFAs from fermentation of a high fibre diet can decrease IgG titres in the blood ${ }^{254}$. In addition, SCFAs differentially affect immune responses depending on the context. Under steady-state conditions, SCFAs act directly on peripheral $\mathrm{T}$ cells to promote differentiation to $\mathrm{IL}^{-10^{+}}$regulatory cells, whereas inflammatory conditions prompt $\mathrm{T}$ cell polarization to a $\mathrm{T}_{\mathrm{H}} 1$ and $\mathrm{T}_{\mathrm{H}} 17$ effector phenotype ${ }^{180}$. Analogously, it is possible SCFAs may promote the development of $\mathrm{T}$ follicular regulatory ( $\left.\mathrm{T}_{\mathrm{FR}}\right)$ cells, which can develop from TREG cells in the $\mathrm{LN}^{255}$ and limit the magnitude of the GC response $^{256}$. However, the impact of SCFAs on $\mathrm{T}_{\mathrm{FH}}$ cell development has not yet 
been investigated. Thus, the immunomodulatory influences of SCFAs are complex and further investigation is required to elucidate their impact on antibody production. Further analysis of the viral RNA load following infection in this experiment is required and may reveal protection against infection with high fibre feeding.

In mice fed the propolis formulation, a clear trend towards reduced viral RNA was observed following infection, despite no improvements in virus-specific antibody production. Statistical analysis could not be performed as viral RNA was non-detectable in a number of mice. $\gamma$-cyclodextrin is used for microencapsulation of the propolis to enhance bioavailability and constitutes $75 \%$ of the propolis formulation. Cyclodextrins are oligosaccharides that are potential prebiotics and, as $\gamma$-cyclodextrin has been shown to alter faecal microbial populations ${ }^{257}$, it may be influencing the microbiota in this study. To determine if beneficial influence of the propolis formulation was due to the action of $\gamma$-cyclodextrin, future investigations should also analyse the effect of $\gamma$-cyclodextrin alone. This investigation does not demonstrate that the beneficial effect of propolis formulation on influenza vaccine responses is mediated through modulation of the microbiota community structure or its function. To prove a causative role of changes to the microbiota in improved immunity, it would be necessary to transfer the gut microbiota from propolis formulation treated mice to untreated mice and observe a transfer of the phenotype.

These experiments also demonstrate that serum IgG may not be a good correlate of protection against infection in this model. TIV vaccination induces virus-specific IgG in humans and this is a direct correlate of protection against infection $^{99}$. In contrast, mice administered propolis formulation exhibited increased protection from infection following vaccination despite no increase in vaccine-induced antibody, whilst mice administered kiwifruit had reduced 
antibody production compared to controls yet protection against infection was comparable. Vaccination may induce other effector functions and immune cell populations to protect against infection, such as $\mathrm{CD}^{+} \mathrm{T}$ cells. Indeed, it has been demonstrated that whole inactivated influenza vaccines are able to induce CD8 ${ }^{+}$ T cell responses in mice ${ }^{258,259}$, although TIV lacks the ability to induce robust CD8 ${ }^{+}$ T cell responses in humans. Despite the fact that correlates of protection against infection could be broader than those investigated currently, the measurement of virus-specific IgG allowed assessment of the efficacy of nutritional interventions to improve antibody responses. Influenza vaccine efficacy is currently measured by seroconversion, therefore a change in the definition of a correlate of protection to influenza vaccination would be required if health claims were to be made on data showing alteration of any other immune parameter.

\subsection{Conclusions}

In this chapter, a mouse model of human parenteral TIV vaccination and subsequent infection was established in which both antibody responses and protection against infection were suboptimal. Using this model, no appreciable improvement in antibody production to vaccination or protection against subsequent infection were observed with administration of kiwifruit, B. animalis or alterations to the fibre content of the murine diet. Interestingly, administration of a propolis formulation tended to improve protection against infection of vaccinated mice, with some mice exhibiting no detectable viral RNA in the lung, despite no enhancement in vaccine-induced IgG antibody.

Whilst administration of the propolis formulation improved immunity to influenza this investigation has not confirmed this was a consequence of changes 
in the composition or function of the gut microbiota communities and further evaluation of the involvement of the microbiota in this phenotype are warranted. 

5 General discussion 


\subsection{Summary of findings}

The aim of this thesis was to examine the role of the microbiota in the humoral immune response to influenza vaccination and further investigate if microbiota-targeted nutritional interventions could enhance immune responses in a murine model.

Administration of broad-spectrum $\mathrm{ABX}$ was shown to alter the composition of the gut microbiota and lead to severely reduced antibody responses to influenza vaccination. Concurrently, $A B X$ treatment led to significant impairments in many cell compartments crucial to the humoral immune response. The frequency and activation of a number of myeloid cell subsets in the dLN were reduced, both prior to and post-vaccination. In addition, there were significantly fewer $\mathrm{T}_{\mathrm{FH}}$ cells in the dLN following vaccination in ABX-treated mice and generation of the GC B cell response was impaired at multiple stages. Collectively, this data demonstrates that altering microbial signals with $A B X$ treatment leads to abnormalities in multiple cell compartments that are instrumental to the humoral immune response and, consequently, vaccine-induced antibody production.

Having established the importance of intact microbial signals in production of a robust antibody response to influenza vaccination, a murine model of a suboptimal response to human parenteral TIV vaccination was established to trial the benefit of potential microbiota-altering nutritional interventions to enhance immune responses in this model. Administration of kiwifruit, $B$. animalis or alterations to the fibre content of the murine diet did not improve immune responses to influenza vaccination or infection. Promisingly, an appreciable improvement was observed in viral control following 
administration of a propolis formulation in vaccinated mice, despite antibody production to vaccination remaining unchanged. 


\subsection{The microbiota influences multiple cell populations in the influenza vaccine response}

Whilst there is substantial evidence that the gut microbiota promotes systemic inflammatory responses in a number of contexts, the mechanism by which gut commensal-derived signals control innate and adaptive immunity at sites beyond the gut, such as in antibody responses to influenza vaccination, are incompletely understood.

In this investigation, administration of $A B X$ to deplete the microbiota resulted in severe impairments in antibody production to TIV vaccination, in line with numerous reports showing robust immune responses to infection $157,212,229,260,261$ and vaccination ${ }^{127,158}$ are dependent on gut microbial signals. A major question that remains largely unexplained in this study and throughout the field is the mechanism by which the gut microbiota influences immune processes at gut-distal sites. One major theory that has been proposed is that, while it is unlikely that intact bacteria enter systemic circulation ${ }^{262}$, microbial components or metabolites from the gut lumen may translocate across the mucosa into the circulation and effect distal immune cells. This has been directly demonstrated by Clarke and colleagues, who showed that peptidoglycan from the murine microbiota is able to enter the circulation, reaching the $\mathrm{BM}$ and enhancing the killing capacity of neutrophils ${ }^{260}$. Furthermore, this theory is supported by the observation that immune hyporesponsiveness in GF or ABX-treated mice can be rescued by infusion of heat-treated serum ${ }^{159}$ or PRR ligands ${ }^{156,158}$. In addition, gut microbiota-derived metabolites such as SCFAs are absorbed into the blood and contribute to many crucial immune processes, including haematopoesis ${ }^{159,163}$ and directing T cell differentiation ${ }^{180,181,263}$. An alternate theory is that immune cells are influenced by microbial products in the gut and migrate to the periphery where they prompt an augmented immune response. This is supported by 
findings that in a model of autoimmune arthritis, the gut mucosa adherent microbes, segmented filamentous bacteria (SFB), promote differentiation of autoreactive $T_{F H}$ in the Peyer's patches of the intestine, which are able to egress to systemic sites, such as the spleen and foot dLN, and are essential for the production of auto-reactive antibodies and the arthritic phenotype ${ }^{264}$. Indeed, evidence suggests that auto-reactive T cells specific for pancreatic islet cells in a model of type-1 diabetes originate in the gut and express gut-homing receptors ${ }^{265,266}$. It has also been proposed that DCs and $\mathrm{M} \varphi$ acquire microbial antigen from the gut and transport it to peripheral lymph nodes where it could influence immune cells ${ }^{267}$, although evidence for this hypothesis is lacking. Oh and colleagues confirmed that vaccine-specific B cells were not present in the gut or associated lymphoid tissues following influenza vaccination ${ }^{158}$ and it has yet to be demonstrated if microbially-influenced immune cells from the gut migrate to the periphery to augment antibody production to influenza vaccination.

Notably, whilst this investigation focused on the gut-resident microbiota, it is likely that $\mathrm{ABX}$ treatment is altering microbial communities at other sites. Tissue-resident commensal microbes have been shown to influence immunity in proximal tissues. For example, skin microbes control differentiation of local $\mathrm{T}$ cells to protect against cutaneous parasitic infection ${ }^{268}$. Therefore, it is possible in this model that $\mathrm{ABX}$ are also modulating microbes at other sites, such as the skin, to influence the antibody response. To ensure this was a gut microbiota-specific effect, neomycin could be administered in isolation - an antibiotic not readily absorbed through the intestine, therefore only eliminating gut microbes ${ }^{157,269}$.

Development of class-switched, high affinity antibody-secreting B cells to infection and vaccination is a 'multi-player' process in lymphoid organs. TFH cells require concerted interactions with myeloid and B cells for their development, while signals from both myeloid cells and $\mathrm{CD}^{+} \mathrm{T}$ cells are required for $\mathrm{B}$ cell 
expansion and differentiation. The data presented in this thesis suggests that reducing signals from the commensal microbiota with antibiotics leads to a reduction in the homeostatic frequency and activation status of many myeloid populations at gut-distal sites. This results in reduced quantity and quality of cells able to immediately respond upon vaccination, reflected in severely compromised myeloid populations three days following TIV vaccination. This is in agreement with a number of previous investigations showing the requirement for an intact microbiota in maintaining systemic immune homeostasis, maintaining steady-state haematopoesis ${ }^{159,163}$, and the constitutive production of chemokines to promote egress of myeloid cell precursors from the BM to sustain immune cell populations throughout the body ${ }^{228}$. Furthermore, deprivation of microbial signals impairs homeostatic DC distribution and activation, as well as their ability to migrate to the dLN following influenza infection ${ }^{157}$ and $\mathrm{M} \varphi$ from ABX-treated mice have major defects in key antiviral transcriptional programmes and are less responsive to influenza virus infection ${ }^{212}$. Together with previous investigations, the data presented suggests that homeostatic signalling from an intact microbiota calibrates myeloid cells to respond effectively to vaccination and this likely is contributing to optimal humoral immunity to TIV.

In this investigation, $\mathrm{ABX}$ treatment also led to a reduction in the number of $\mathrm{T}_{\mathrm{FH}}$ cells in the dLN following vaccination. Development of $T_{F H}$ cells is dependent on signals from myeloid cells and B cells. These findings do not determine if TFH cell development following TIV directly relies on microbiota signals or if reduced TFH cell numbers are due to a lack of signals from myeloid or B cell populations. Given that microbiota-derived molecules are able to directly drive $\mathrm{T}$ cell polarisation $^{180}$ and that gut-primed T cells are able to migrate to systemic sites ${ }^{264-}$ ${ }^{266}$, it is conceivable that defects may be T cell intrinsic. Nevertheless, because such profound changes are seen to affect dLN myeloid population number and 
expression of costimulatory molecules, it is also highly likely that $\mathrm{T}$ cells are receiving reduced antigen-presentation, co-stimulation and cytokine signals from DCs and $\mathrm{M} \varphi$, and this may account for reduced $\mathrm{T}_{\mathrm{FH}}$ cells following vaccination.

B cell differentiation following vaccination was impaired at multiple stages in ABX-treated mice, with reductions in GC B cells, CS B cells and PCs and alterations to the microbiota may be directly signalling on B cells to account for this. Microbial components can influence B cells directly in a number of ways. B cells express TLR-1, 2, 4, 6, 7 and $9^{270}$ and ligation of TLRs by microbial products increases B cell survival, as well as antigen presentation and antibody production $^{69,234}$. At steady-state, binding of lipopolysaccharide (LPS) from the microbiota to B cells directly induces CSR to $\operatorname{IgA}$ in the gut ${ }^{271}$. Bacterial metabolites, such as SCFAs also enhance B cell development and antibody production. Antibody production is an energy demanding process and SCFAs have been shown to be directly utilised by peripheral B cells to produce acetyl CoA for fatty acid synthesis, metabolically fueling antibody production, as well as simultaneously inhibiting HDACs for expression of genes enhancing $\operatorname{CSR}^{175}$. Therefore, the detrimental effects of $\mathrm{ABX}$ treatment on $\mathrm{B}$ cell development following vaccination may be due to alterations to TLR signalling on B cells directly, or deprivation of certain microbial metabolites, such as SCFAs. Thus, whilst $\mathrm{ABX}$ treatment impacted multiple immune cell populations both at baseline and following vaccination, ultimately leading to defective $B$ cell development and impaired antibody production, further investigation is required to elucidate the particular immune cell compartment alterations that are causative of this phenotype. 


\subsection{Enhancing influenza vaccine efficacy with functional foods}

Antibody responses induced in individuals administered the influenza vaccine can vary up to 100 -fold ${ }^{115}$, with particularly poor responses mounted in young children and the elderly ${ }^{83}$, for reasons that have not been entirely elucidated. As discussed earlier and demonstrated in this thesis, there is emerging evidence of the pervasive influence of the gut microbiota on influenza vaccine responses ${ }^{115,158}$. The microbiota of infants ${ }^{125}$ and the elderly ${ }^{124}$ are divergent from those of the general population and highly sensitive to change, which possibly contributes to the aberrant immune responses to influenza vaccination in these groups. Effective protection from influenza is particularly important in these populations and microbiota-targeted dietary interventions may be a safe and effective manner in which to improve vaccine responses in these vulnerable groups.

No improvement in antibody production to influenza vaccination nor protection from subsequent infection was observed following administration of kiwifruit, B. animalis or by altering the fibre content of the murine diet. The response to specific microbiota interventions may depend on the baseline microbiota composition. For example, following oral administration of the probiotic, $B$. longum AH1206, long-term incorporation of the bacteria into the host microbiota only occurred in individuals already harboring a lower abundance of the $B$. longum species ${ }^{251}$. Furthermore, it has been found that when a low diversity microbiota from individuals consuming a typical 'western' diet was transplanted to GF mice, mice were only able to recover microbiota diversity if they were both cohoused with mice transplanted with microbiota from humans consuming a nutritious diet and simultaneously consuming the nutritious $\operatorname{diet}^{272}$. This illustrates that dietary interventions or probiotic therapies may not be 
effective therapies alone, certain species may have to be introduced with probiotics in conjunction with dietary interventions in order to beneficially modulate the microbiota and enhance immune responses to influenza vaccination. Of note, mice fed nutritional interventions were maintained on a lower fibre, higher fat and refined 'western' diet, which is known to reduce microbiota diversity ${ }^{273}$, and the baseline microbiota composition whilst on this diet may not have favoured beneficial changes in the microbiota. Furthermore, it would be interesting to test if these nutritional interventions were able to mediate the negative effects of $\mathrm{ABX}$ on antibody responses to influenza vaccination.

\subsection{The future of microbiota-targeted therapies}

Despite significant efforts to determine effective microbiota-targeted therapies to improve outcomes in immune-related diseases, effective therapies remain elusive for enhancing immune responses in human populations.

A major issue that remains is the high variability of gut microbiota composition that exists between individuals ${ }^{128}$, and, in turn, the disparate responsiveness of microbial communities to microbiota-targeted interventions ${ }^{251,274}$. The difficulty in identifying true probiotic or prebiotic candidates to improve disease outcomes may lie in effects being masked in trials by non-responders. Therefore, it is clear that population stratification based on structural or functional features of the microbiota will be necessary in order to predict the response to nutritional interventions and personalise microbiota-targeted therapies for enhancing immunity. Demonstrating the validity of this approach, Zeevi et al. observed high variability in glycaemic responses between individuals following intake of the same meals ${ }^{275}$. They showed that by integrating data on an individual's gut microbiota, genetics and environmental factors into a machine-learning algorithm, they were able to more accurately predict dietary recommendations 
to lower postprandial glycaemic responses than a clinical dietician ${ }^{275}$. Approaches of this kind, focussed on the microbiome of the individual, may allow the design of personalised diets to augment gut microbiota functions to achieve optimal immune responses.

Another key issue is the lack of evidence of specific bacteria or metabolites that correspond to desired immune responses in human cohorts. Efforts to determine microbiota signatures that are associated with health and disease have up until recently been focussed on phylogenetic classification of the microbiota and have had limited success in translation to the clinic. Phylogenetic classification may be an irrelevant way to classify bacteria based on their immunomodulatory properties. A recent investigation analysed the effect of GF mice mono-colonised with different bacterial species on innate and adaptive immune responses, showing that there was no relationship between bacterial phylogeny and influences on the host immune system ${ }^{276}$. Integration of the range of microbiome analysis technologies, including metagenomics, metatranscriptomics and metabolomics will allow a more complete picture of both structure and function of microbial communities. This will allow classification of gut microbiota features in states of health and disease in order for rational design of effective microbiota-targeted therapies.

Continued efforts to understand what compositional and functional characteristics are associated with desired immune outcomes, as well as stratification of individuals, will lead to the development of microbiota-targeted therapies to improve disease outcomes, including the enhancement of vaccine responses. 


\subsection{Future directions}

This report has focused on primary responses to influenza vaccination. It should be noted, however, that human recipients of the vaccine will often have had more than one influenza vaccination or been exposed naturally to multiple viral strains. Accordingly, although circulating viral strains vary seasonally, cross-reactive immunity can occur between similar strains and thus immune responses to vaccination may be secondary, or memory, responses ${ }^{121}$. Therefore, in order for this research to have greater translational impact in humans, future investigations should analyse secondary responses to vaccination to assess if the microbiota also influences recall of influenza-specific memory.

Deprivation of microbial signals with long-term ABX treatments has been shown to impact haematopoesis ${ }^{159,229}$. Flow cytometric analysis of BM precursors should be performed to determine if short-term $\mathrm{ABX}$ administration is having substantial effects on haematopoesis, leading to the broad reductions in the frequency of myeloid cell populations that was observed.

In this thesis, the $\mathrm{ABX}$ treatment significantly altered gut microbiota communities and, in turn, impacted humoral immunity to vaccination. It would be interesting to determine if this phenotype was due to $A B X$-induced alterations to PRR ligands from the microbiota, or due to changes in microbiota-produced metabolites, such as SCFAs. To do so, alongside ABX-administration, mice should be orally administered SCFAs or autoclaved caecal contents from SPF mice in order to restore microbiota-derived MAMPS, and determining whether either of these stimuli restores antibody responses.

Following vaccination, mice orally administered the propolis formulation exhibited a reduction in viral RNA load independent of any improvement in virus-specific antibodies. To determine immunological parameters that may 
have been altered in mice administered the propolis formulation, an array analysis of an extensive panel of cytokines should be performed on serum and lung homogenates following infection. This would give a broad indication of immune parameters stimulated by the propolis formulation that may be responsible for improved viral protection.

Furthermore, to ascertain if the improvements observed in anti-viral responses following propolis formulation administration were mediated by alterations to the microbiota, transferral of microbial communities from propolis formulation fed mice would need to transfer the phenotype. To test this, faecal transplantation experiments could be performed, where gut contents from propolis formulation fed mice are administered IG to control mice in order to transfer microbial communities. Alternately, as mice are coprophagic, propolis formulation fed mice could be cohoused with controls which would also result in transferal of microbial communities, albeit in a less controlled manner ${ }^{277}$. 


\subsection{Final conclusions}

The evidence outlined in this thesis demonstrates the involvement of the gut microbiota in robust antibody responses to influenza vaccination in mice and that immune responses may be able to be improved through nutritional interventions. Considering the emerging links between the commensal microbiota and a wide range of immune-related diseases, this area of research is vital and microbiota-targeted therapies are likely to be key in combatting a range of diseases in the future.

To summarise:

1) Short-term administration of broad-spectrum $A B X$ alters the composition of the gut microbiota and leads to severely reduced antibody responses to TIV vaccination.

2) $A B X$ significantly reduce the number and activation status of myeloid cell subsets in the dLN at steady-state and following vaccination. This is concurrent with a reduction in $\mathrm{T}_{\mathrm{FH}}$ cell numbers and a deficiency in B cells at multiple stages of differentiation following TIV vaccination

3) UV inactivation of a mouse-adaptive influenza virus serves as an appropriate model of a suboptimally-responding human population to parenteral TIV vaccination and infection.

4) Administration of kiwifruit, B. animalis or alterations to the fibre content of murine diet does not improve antibody production to vaccination or protection against subsequent infection. A propolis-cyclodextrin formulation leads to improved protection against infection in vaccinated mice following infection, without influencing vaccine-induced antibody production. 


\section{Supplemental figures}

Supplementary table 1: qPCR primers

\begin{tabular}{|c|c|c|c|c|}
\hline Target & Primer & Sequence $\left(5^{\prime}-3^{\prime}\right)$ & Source & Supplier \\
\hline Bacteroidetes & $\begin{array}{l}\text { Bac960F } \\
\text { Bac1100R }\end{array}$ & $\begin{array}{l}\text { GTTTAATTCGATGATACGCGAG } \\
\text { TTAAGCCGACACCTCACGG }\end{array}$ & 215 & Sigma \\
\hline Firmicutes & $\begin{array}{l}\text { Firm934F } \\
\text { Firm1060R }\end{array}$ & $\begin{array}{l}\text { GGAGCATGTGGTTTAATTCGAAGCA } \\
\text { AGCTGACGACAACCATGCAC }\end{array}$ & 215 & Sigma \\
\hline Actinobacteria & $\begin{array}{l}\text { Act664F } \\
\text { Act941R }\end{array}$ & $\begin{array}{c}\text { TGTAGCGGTGGAATGCGC } \\
\text { AATTAAGCCACATGCTCCGCT }\end{array}$ & 215 & Sigma \\
\hline Candidatus Saccharibacteria & $\begin{array}{l}\text { Sac1031F } \\
\text { Sac1218R }\end{array}$ & $\begin{array}{l}\text { AAGAGAACTGTGCCTTCGG } \\
\text { GCGTAAGGGAAATACTGACC }\end{array}$ & 215 & Sigma \\
\hline Deferribacteres & $\begin{array}{l}\text { Defer1115F } \\
\text { Defer1265R }\end{array}$ & $\begin{array}{l}\text { CTATTTCCAGTTGCTAACGG } \\
\text { GAGATGCTTCCСTCTGATTATG }\end{array}$ & 215 & Sigma \\
\hline
\end{tabular}




\begin{tabular}{|c|c|c|c|c|}
\hline Verrucomicrobia & $\begin{array}{l}\text { Ver1165F } \\
\text { Ver1263R }\end{array}$ & $\begin{array}{l}\text { TCATGTCAGTATGGCCCTTAT } \\
\text { CAGTTTTCAGGATTTCCTCCGCC }\end{array}$ & 215 & Sigma \\
\hline Tenericutes & $\begin{array}{l}\text { Ten662F } \\
\text { Ten862R }\end{array}$ & $\begin{array}{l}\text { ATGTGTAGCGGTAAAATGCGTAA } \\
\text { CATACTTGCGTACGTACTACT }\end{array}$ & 215 & Sigma \\
\hline$\beta$ proteobacteria & $\begin{array}{l}\text { Beta979F } \\
\text { Beta1130R }\end{array}$ & $\begin{array}{l}\text { AACGCGAAAAACCTTACCTACC } \\
\text { TGCCCTTTCGTAGCAACTAGTG }\end{array}$ & 215 & Sigma \\
\hline$\varepsilon$ proteobacteria & $\begin{array}{l}\text { Epsilon940F } \\
\text { Epsilon1129R }\end{array}$ & $\begin{array}{l}\text { TAGGCTTGACATTGATAGAATC } \\
\text { CTTACGAAGGCAGTCTCCTTA }\end{array}$ & 215 & Sigma \\
\hline$\gamma$ proteobacteria & $\begin{array}{l}\text { Gamma877F } \\
\text { Gamma1066R }\end{array}$ & $\begin{array}{l}\text { GCTAACGCATTAAGTACCCCG } \\
\text { GCCATGCAGCACCTGTCT }\end{array}$ & 215 & Sigma \\
\hline Universal $16 \mathrm{~S}$ & $\begin{array}{l}926 \mathrm{~F} \\
1062 \mathrm{R}\end{array}$ & $\begin{array}{l}\text { AAACTCAAATGAATTGACGG } \\
\text { CTCACAACACGAGCTGAC }\end{array}$ & 215 & Sigma \\
\hline
\end{tabular}


(A)

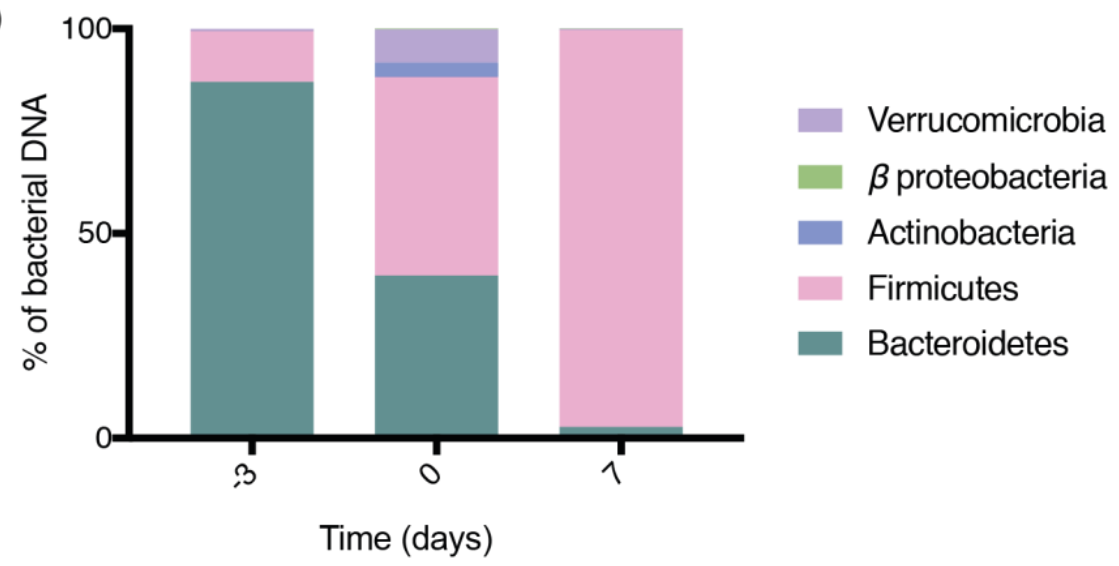

(B)

\begin{tabular}{|c|c|c|c|c|}
\hline Time (days) & Sample & $\begin{array}{c}\text { Nucleic acid } \\
(\mathbf{n g} / \boldsymbol{\mu} \mathbf{L})\end{array}$ & A260/A280 & A260/A230 \\
\hline D-3 & 1 & 424.237 & 2.013 & 2.101 \\
\hline & 2 & 211.518 & 1.97 & 1.47 \\
\hline & 3 & 239.099 & 1.952 & 1.792 \\
\hline & 4 & 327.263 & 1.969 & 1.686 \\
\hline D0 & 1 & 31.202 & 1.815 & 0.514 \\
\hline & 2 & 29.702 & 1.797 & 0.573 \\
\hline & 3 & 40.018 & 1.793 & 0.423 \\
\hline D7 & 1 & 67.329 & 1.842 & 0.742 \\
\hline & 2 & 23.867 & 1.976 & 0.367 \\
\hline & 3 & 64.815 & 1.94 & 0.411 \\
\hline & 4 & 57.212 & 1.81 & 0.454 \\
\hline
\end{tabular}

Supplementary figure 1: qPCR analysis of faecal DNA in antibiotic-treated mice. C57BL/6 mice received $\mathrm{ABX}$ ad libitum in the drinking water for three days prior to initial vaccination until seven days following vaccination. (A) Faecal-resident bacterial frequencies within a cage of mice throughout $\mathrm{ABX}$ treatment was determined by qPCR using $16 \mathrm{~S}$ rRNA gene-targeted groupspecific primers for Bacteroidetes, Firmicutes, Proteobacteria, Actinobacteria, Verrucomicrobia, Candidatus Saccharibacteria, Deferribacteres and Tenericutes, and proportions were determined against a universal $16 \mathrm{~S}$ sequence. Proportions of bacteria are presented as the group mean. (B) Nanodrop quality analysis of faecal DNA. Data shown is $n=3-5$ per group. 



\section{References}

1. Murphy K. Janeways Immunobiology. 8 ed. Garland Science; 2012.

2. Iwasaki A, Medzhitov R. Toll-like receptor control of the adaptive immune responses. Nat Immunol. 2004;5(10):987-995.

3. Janeway CA. Approaching the asymptote? Evolution and revolution in immunology. Cold Spring Harb Symp Quant Biol. 1989;54:1-13.

4. Schaefer L. Complexity of danger: the diverse nature of damageassociated molecular patterns. J Biol Chem. 2014;289(51):35237-35245.

5. Bassing $\mathrm{CH}$, Swat W, Alt FW. The mechanism and regulation of chromosomal V(D)J recombination. Cell. 2002;109:S45-55.

6. Lanzavecchia A, Sallusto F. From synapses to immunological memory: The role of sustained T cell stimulation. Curr Opin Immunol. 2000;12(1):92-98.

7. De Silva NS, Klein U. Dynamics of B cells in germinal centres. Nat Rev Immunol. 2015;15(3):137-148.

8. Trapani JA, Smyth MJ. Functional significance of the perforin/granzyme cell death pathway. Nat Rev Immunol. 2002;2(10):735-747.

9. Stout RD, Bottomly K. Antigen-specific activation of effector macrophages by IFN-gamma producing (TH1) T cell clones. Failure of IL-4-producing (TH2) T cell clones to activate effector function in macrophages. J Immunol. 1989;142(3):760-765.

10. Walker JA, McKenzie ANJ. TH2 cell development and function. Nat Rev Immunol. 2018;18(2):121-133.

11. Park H, Li Z, Yang XO, et al. A distinct lineage of CD4 T cells regulates tissue inflammation by producing interleukin 17. Nat Immunol. 2005;6(11):1133-1141.

12. Boehmer von H. Mechanisms of suppression by suppressor T cells. Nat Immunol. 2005;6(4):338-344.

13. Crotty S. Follicular helper CD4 T cells (TFH). Annu Rev Immunol. 2011;29(1):621-663.

14. Harris NL, Ronchese F. The role of B7 costimulation in T-cell immunity. 
Immunol Cell Biol. 1999;77(4):304-311.

15. Yoshida R, Nagira M, Kitaura M, Imagawa N, Imai T, Yoshie O.

Secondary lymphoid-tissue chemokine is a functional ligand for the CC chemokine receptor CCR7. J Biol Chem. 1998;273(12):7118-7122.

16. Walsh KP, Mills KHG. Dendritic cells and other innate determinants of T helper cell polarisation. Trends Immunol. 2013;34(11):521-530.

17. Davies DR, Chacko S. Antibody Structure. Acc Chem Res. 1993;26(8):421-427.

18. Teng G, Papavasiliou FN. Immunoglobulin Somatic Hypermutation. Annu Rev Genet. 2007;41(1):107-120.

19. Schroeder HW Jr., Cavacini L. Structure and function of immunoglobulins. J Allergy Clin Immunol. 2010;125(2):S41-S52.

20. Stavnezer J, Guikema JEJ, Schrader CE. Mechanism and Regulation of Class Switch Recombination. Annu Rev Immunol. 2008;26(1):261-292.

21. Abney ER, Cooper MD, Kearney JF, Lawton AR, Parkhouse RM. Sequential expression of immunoglobulin on developing mouse B lymphocytes: a systematic survey that suggests a model for the generation of immunoglobulin isotype diversity. J Immunol. 1978;120(6):2041-2049.

22. Heyman B, Pilström L, Shulman MJ. Complement activation is required for IgM-mediated enhancement of the antibody response. J Exp Med. 1988;167(6):1999-2004.

23. Ferrante A, Beard LJ, Feldman RG. IgG subclass distribution of antibodies to bacterial and viral antigens. Pediatr Infect Dis J. 1990;9(8):S16-24.

24. Bindon CI, Hale G, Brüggemann M, Waldmann H. Human monoclonal IgG isotypes differ in complement activating function at the level of C4 as well as C1q. J Exp Med. 1988;168(1):127-142.

25. Macpherson AJ, McCoy KD, Johansen F-E, Brandtzaeg P. The immune geography of $\operatorname{IgA}$ induction and function. Mucosal Immunol. 2008;1(1):11-22.

26. Wijburg OLC, Uren TK, Simpfendorfer K, Johansen F-E, Brandtzaeg P, Strugnell RA. Innate secretory antibodies protect against natural Salmonella typhimurium infection. J Exp Med. 2006;203(1):21-26. 
27. Renegar KB, Small PA, Boykins LG, Wright PF. Role of IgA versus IgG in the Control of Influenza Viral Infection in the Murine Respiratory Tract. 2004;173(3):1978-1986.

28. Galli SJ, Tsai M. IgE and mast cells in allergic disease. Nat Med. 2012;18(5):693-704.

29. Itano AA, Jenkins MK. Antigen presentation to naive CD4 T cells in the lymph node. Nat Immunol. 2003;4(8):733-739.

30. Pape KA, Catron DM, Itano AA, Jenkins MK. The Humoral Immune Response Is Initiated in Lymph Nodes by B Cells that Acquire Soluble Antigen Directly in the Follicles. Immunity. 2007;26(4):491-502.

31. Roozendaal R, Mempel TR, Pitcher LA, et al. Conduits Mediate Transport of Low-Molecular-Weight Antigen to Lymph Node Follicles. Immunity. 2009;30(2):264-276.

32. Phan TG, Grigorova I, Okada T, Cyster JG. Subcapsular encounter and complement-dependent transport of immune complexes by lymph node B cells. Nat Immunol. 2007;8(9):992-1000.

33. Gonzalez SF, Lukacs-Kornek V, Kuligowski MP, et al. Capture of influenza by medullary dendritic cells via SIGN-R1 is essential for humoral immunity in draining lymph nodes. Nat Immunol. 2010;11(5):427-434.

34. Allenspach EJ, Lemos MP, Porrett PM, Turka LA, Laufer TM. Migratory and lymphoid-resident dendritic cells cooperate to efficiently prime naive CD4 T cells. Immunity. 2008;29(5):795-806.

35. Gretz JE, Norbury CC, Anderson AO, Proudfoot AE, Shaw S. Lymphborne chemokines and other low molecular weight molecules reach high endothelial venules via specialized conduits while a functional barrier limits access to the lymphocyte microenvironments in lymph node cortex. J Exp Med. 2000;192(10):1425-1440.

36. Sixt M, Kanazawa N, Selg M, et al. The Conduit System Transports Soluble Antigens from the Afferent Lymph to Resident Dendritic Cells in the T Cell Area of the Lymph Node. Immunity. 2005;22(1):19-29.

37. Batista FD, Harwood NE. The who, how and where of antigen presentation to B cells. Nat Rev Immunol. 2009;9(1):15-27.

38. Andrian von UH, Mempel TR. Homing and cellular traffic in lymph 
nodes. Nat Rev Immunol. 2003;3(11):867-878.

39. Marchesi VT, Gowans JL. The migration of lymphocytes through the endothelium of venules in lymph nodes: An electron microscope study. Proc R Soc Lond B Biol Sci. 1964;159(975):283-290.

40. Miller MJ, Wei SH, Parker I, Cahalan MD. Two-photon imaging of lymphocyte motility and antigen response in intact lymph node. Science. 2002;296(5574):1869-1873.

41. Kerfoot SM, Yaari G, Patel JR, et al. Germinal center B cell and T follicular helper cell development initiates in the interfollicular zone. Immunity. 2011;34(6):947-960.

42. Okada T, Miller MJ, Parker I, et al. Antigen-engaged B cells undergo chemotaxis toward the $\mathrm{T}$ zone and form motile conjugates with helper T cells. PLoS Biol. 2005;3(6):150.

43. Foy TM, Laman JD, Ledbetter JA, Aruffo A, Claassen E, Noelle RJ. gp39-CD40 interactions are essential for germinal center formation and the development of B cell memory. J Exp Med. 1994;180(1):157-163.

44. Taylor JJ, Pape KA, Jenkins MK. A germinal center-independent pathway generates unswitched memory B cells early in the primary response. J Exp Med. 2012;209(3):597-606.

45. Ho F, Lortan JE, MacLennan IC, Khan M. Distinct short-lived and longlived antibody-producing cell populations. Eur J Immunol. 1986;16(10):1297-1301.

46. Goenka R, Barnett LG, Silver JS, et al. Cutting edge: dendritic cellrestricted antigen presentation initiates the follicular helper $\mathrm{T}$ cell program but cannot complete ultimate effector differentiation. J Immunol. 2011;187(3):1091-1095.

47. Linterman MA, Liston A, Vinuesa CG. T-follicular helper cell differentiation and the co-option of this pathway by non-helper cells. Immunol Rev. 2012;247(1):143-159.

48. Linterman MA, Denton AE, Divekar DP, et al. CD28 expression is required after $\mathrm{T}$ cell priming for helper $\mathrm{T}$ cell responses and protective immunity to infection. Elife. 2014;3.

49. Barnett LG, Simkins HMA, Barnett BE, et al. B cell antigen presentation in the initiation of follicular helper $T$ cell and germinal center 
differentiation. J Immunol. 2014;192(8):3607-3617.

50. Ettinger R, Sims GP, Fairhurst A-M, et al. IL-21 induces differentiation of human naive and memory $\mathrm{B}$ cells into antibody-secreting plasma cells. J Immunol. 2005;175(12):7867-7879.

51. Good KL, Bryant VL, Tangye SG. Kinetics of human B cell behavior and amplification of proliferative responses following stimulation with IL21. J Immunol. 2006;177(8):5236-5247.

52. Cervenak L, Magyar A, Boja R, László G. Differential expression of GL7 activation antigen on bone marrow $B$ cell subpopulations and peripheral B cells. Immunol Lett. 2001;78(2):89-96.

53. Muramatsu M, Kinoshita K, Fagarasan S, Yamada S, Shinkai Y, Honjo T. Class switch recombination and hypermutation require activationinduced cytidine deaminase (AID), a potential RNA editing enzyme. Cell. 2000;102(5):553-563.

54. Mandel TE, Phipps RP, Abbot AP, Tew JG. Long-term antigen retention by dendritic cells in the popliteal lymph node of immunized mice. Immunology. 1981;43(2):353-362.

55. Schwickert TA, Victora GD, Fooksman DR, et al. A dynamic T celllimited checkpoint regulates affinity-dependent B cell entry into the germinal center. J Exp Med. 2011;208(6):1243-1252.

56. Shulman Z, Gitlin AD, Weinstein JS, et al. Germinal centers: Dynamic signaling by $\mathrm{T}$ follicular helper cells during germinal center $\mathrm{B}$ cell selection. Science. 2014;345(6200):1058-1062.

57. Xu Z, Zan H, Pone EJ, Mai T, Casali P. Immunoglobulin class-switch DNA recombination: induction, targeting and beyond. Nat Rev Immunol. 2012;12(7):517-531.

58. Rothman P, Lutzker S, Cook W, Coffman R, Alt FW. Mitogen plus interleukin 4 induction of C epsilon transcripts in B lymphoid cells. J Exp Med. 1988;168(6):2385-2389.

59. Slifka MK, Antia R, Whitmire JK, Ahmed R. Humoral immunity due to long-lived plasma cells. Immunity. 1998;8(3):363-372.

60. Yu X, Tsibane T, McGraw PA, et al. Neutralizing antibodies derived from the B cells of 1918 influenza pandemic survivors. Nature. August 2008:1-7. 
61. Slifka MK, Matloubian M, Ahmed R. Bone marrow is a major site of long-term antibody production after acute viral infection. J Virol. 1995;69(3):1895-1902.

62. Ahmed R, Gray D. Immunological memory and protective immunity: understanding their relation. Science. 1996;272(5258):54-60.

63. Heesters BA, Myers RC, Carroll MC. Follicular dendritic cells: dynamic antigen libraries. Nat Rev Immunol. 2014;14(7):495-504.

64. Plotkin SA. Vaccination against the major infectious diseases. $C R$ Acad Sci III, Sci Vie. 1999;322(11):943-951.

65. Amanna IJ, Carlson NE, Slifka MK. Duration of humoral immunity to common viral and vaccine antigens. N Engl J Med. 2007;357(19):19031915.

66. Pulendran B, Ahmed R. Immunological mechanisms of vaccination. Nat Immunol. 2011;12(6):509-517.

67. Coffman RL, Sher A, Seder RA. Vaccine adjuvants: putting innate immunity to work. Immunity. 2010;33(4):492-503.

68. Reed SG, Orr MT, Fox CB. Key roles of adjuvants in modern vaccines. Nat Med. 2013;19(12):1597-1608.

69. Kasturi SP, Skountzou I, Albrecht RA, et al. Programming the magnitude and persistence of antibody responses with innate immunity. Nature. 2011;470(7335):543-547.

70. McCluskie MJ, Pryde DC, Gervais DP, et al. Enhancing immunogenicity of a 3'aminomethylnicotine-DT-conjugate anti-nicotine vaccine with CpG adjuvant in mice and non-human primates. Int Immunopharmacol. 2013;16(1):50-56.

71. Marrack P, McKee AS, Munks MW. Towards an understanding of the adjuvant action of aluminium. Nat Rev Immunol. 2009;9(4):287-293.

72. Hutchison S, Benson RA, Gibson VB, Pollock AH, Garside P, Brewer JM. Antigen depot is not required for alum adjuvanticity. FASEB J. 2012;26(3):1272-1279.

73. Calabro S, Tortoli M, Baudner BC, et al. Vaccine adjuvants alum and MF59 induce rapid recruitment of neutrophils and monocytes that participate in antigen transport to draining lymph nodes. Vaccine. 2011;29(9):1812-1823. 
74. Mosca F, Tritto E, Muzzi A, et al. Molecular and cellular signatures of human vaccine adjuvants. Proc Natl Acad Sci USA. 2008;105(30):1050110506.

75. Seubert A, Monaci E, Pizza M, O'Hagan DT, Wack A. The adjuvants aluminum hydroxide and MF59 induce monocyte and granulocyte chemoattractants and enhance monocyte differentiation toward dendritic cells. J Immunol. 2008;180(8):5402-5412.

76. Neill LAJO, Bowie AG. Sensing and signaling in antiviral innate immunity. Current Biology. 2010;20(7):R328-R333.

77. Mata-Haro V, Cekic C, Martin M, Chilton PM, Casella CR, Mitchell TC. The vaccine adjuvant monophosphoryl lipid A as a TRIF-biased agonist of TLR4. Science. 2007;316(5831):1628-1632.

78. Eisenbarth SC, Colegio OR, O'Connor W, Sutterwala FS, Flavell RA. Crucial role for the Nalp3 inflammasome in the immunostimulatory properties of aluminium adjuvants. Nature. 2008;453(7198):1122-1126.

79. World Health Organisation. Influenza (Seasonal). WHO. November 2016.

80. Simonsen L. The global impact of influenza on morbidity and mortality. In: Vol 17. 1999:S3-S10.

81. Huang S. Key findings - SHIVERS.

82. Taubenberger JK, Morens DM. The Pathology of Influenza Virus Infections. Annu Rev Pathol Mech Dis. 2008;3(1):499-522.

83. Boyd M, Clezy K, Lindley R, Pearce R. Pandemic influenza: Clinical issues. Med J Aust. 2006;185(10 Suppl.):S44-47.

84. Palese P, Young JF. Variation of influenza A, B, and C viruses. Science. 1982;215(4539):1468-1474.

85. Moriuchi H, Katsushima N, Nishimura H, Nakamura K, Numazaki Y. Community-acquired influenza $\mathrm{C}$ virus infection in children. J Pediatr. 1991;118(2):235-238.

86. Skehel JJ, Wiley DC. Receptor binding and membrane fusion in virus entry: the influenza hemagglutinin. Annu Rev Biochem. 2000;69(1):531569.

87. Potter CW. Influenza: Perspectives in Medical Virology. Vol 7. 1st ed. 
Elsevier Science; 2002.

88. Shtyrya YA, Mochalova LV, Bovin NV. Influenza virus neuraminidase: structure and function. Acta Naturae. 2009;1(2):26-32.

89. Wagner R, Wolff T, Herwig A, Pleschka S, Klenk HD. Interdependence of hemagglutinin glycosylation and neuraminidase as regulators of influenza virus growth: a study by reverse genetics. J Virol. 2000;74(14):6316-6323.

90. Mitnaul LJ, Matrosovich MN, Castrucci MR, et al. Balanced hemagglutinin and neuraminidase activities are critical for efficient replication of influenza A virus. J Virol. 2000;74(13):6015-6020.

91. Thompson CI, Barclay WS, Zambon MC, Pickles RJ. Infection of human airway epithelium by human and avian strains of influenza A virus. J Virol. 2006;80(16):8060-8068.

92. Wong S-S, Webby RJ. Traditional and new influenza vaccines. Clin Microbiol Rev. 2013;26(3):476-492.

93. Krammer F, Palese P. Advances in the development of influenza virus vaccines. Nat Rev Drug Discov. 2015;14(3):167-182.

94. Chiu C, Ellebedy AH, Wrammert J, Ahmed R. B cell responses to influenza infection and vaccination. Curr Top Microbiol Immunol. 2015;386:381-398.

95. Clements ML, Betts RF, Tierney EL, Murphy BR. Serum and nasal wash antibodies associated with resistance to experimental challenge with influenza A wild-type virus. J Clin Microbiol. 1986;24(1):157-160.

96. Mazanec MB, Coudret CL, Fletcher DR. Intracellular neutralization of influenza virus by immunoglobulin A anti- hemagglutinin monoclonal antibodies. J Virol. 1995;69(2):1339-1343.

97. Burlington DB, Clements ML, Meiklejohn G, Phelan M, Murphy BR. Hemagglutinin-specific antibody responses in immunoglobulin $G, A$, and $\mathrm{M}$ isotypes as measured by enzyme-linked immunosorbent assay after primary or secondary infection of humans with influenza A virus. Infect Immun. 1983;41(2):540-545.

98. Hirota $Y$, Kaji M, Ide S, et al. Antibody efficacy as a keen index to evaluate influenza vaccine effectiveness. Vaccine. 1997;15(9):962-967.

99. Hobson D, Curry RL, Beare AS, Ward-Gardner A. The role of serum 
haemagglutination-inhibiting antibody in protection against challenge infection with influenza A2 and B viruses. Epidemiol Infect. 1972;70(4):767-777.

100. McMichael AJ, Michie CA, Gotch FM, Smith GL, Moss B. Recognition of influenza A virus nucleoprotein by human cytotoxic T lymphocytes. J Gen Virol. 1986;67(4):719-726.

101. Grohskopf LA, Sokolow LZ, Broder KR, et al. Prevention and control of seasonal influenza with vaccines recommendations of the advisory committee on immunization practices - United States, 2016-17 influenza season. MMWR Recomm Rep. 2016;65(5):1-52.

102. World Health Organisation. Recommended composition of influenza virus vaccines for use in the southern hemisphere 2017 influenza season and development of candidate vaccine viruses for pandemic preparedness. September 2016.

103. Gerdil C. The annual production cycle for influenza vaccine. Vaccine. 2003;21(16):1776-1779.

104. Laver WG, Webster RG. Preparation and immunogenicity of a purified influenza virus haemagglutinin and neuraminidase subunit vaccine. Postgrad Med J. 1976;52(608):373-378.

105. La Montagne JR, Noble GR, Quinnan GV. Summary of clinical trials of inactivated influenza vaccine-1978. Clin Infect Dis. 1983.

106. Beyer WE, Palache AM, Osterhaus AD. Comparison of Serology and Reactogenicity between Influenza Subunit Vaccines and Whole Virus or Split Vaccines: A Review and Meta-Analysis of the Literature. Clin Drug Investig. 1998;15(1):1-12.

107. Brokstad KA, Cox RJ, Olofsson J, Jonsson R, Haaheim LR. Parenteral influenza vaccination induces a rapid systemic and local immune response. J Infect Dis. 1995;171(1):198-203.

108. El-Madhun AS, Cox RJ, Søreide A, Olofsson J, Haaheim LR. Systemic and mucosal immune responses in young children and adults after parenteral influenza vaccination. J Infect Dis. 1998;178(4):933-939.

109. Maassab HF, Bryant ML. The development of live attenuated coldadapted influenza virus vaccine for humans. Rev Med Virol. 1999;9(4):237-244. 
110. Belshe RB, Gruber WC, Mendelman PM, et al. Correlates of immune protection induced by live, attenuated, cold-adapted, trivalent, intranasal influenza virus vaccine. J Infect Dis. 2000;181(3):1133-1137.

111. Clements ML, Murphy BR. Development and persistence of local and systemic antibody responses in adults given live attenuated or inactivated influenza A virus vaccine. J Clin Microbiol. 1986;23(1):66-72.

112. Moldoveanu Z, Clements ML, Prince SJ, Murphy BR, Mestecky J. Human immune responses to influenza virus vaccines administered by systemic or mucosal routes. Vaccine. 1995;13(11):1006-1012.

113. Salomon R, Webster RG. The Influenza Virus Enigma. Cell. 2009;136(3):402-410.

114. Treanor JJ, Kotloff K, Betts RF, et al. Evaluation of trivalent, live, coldadapted (CAIV-T) and inactivated (TIV) influenza vaccines in prevention of virus infection and illness following challenge of adults with wild-type influenza A (H1N1), A (H3N2), and B viruses. Vaccine. 1999;18(9-10):899-906.

115. Nakaya HI, Wrammert J, Lee EK, et al. Systems biology of vaccination for seasonal influenza in humans. Nat Immunol. 2011;12(8):786-795.

116. Goodwin K, Viboud C, Simonsen L. Antibody response to influenza vaccination in the elderly: a quantitative review. Vaccine. 2006;24(8):1159-1169.

117. Nakaya HI, Hagan T, Duraisingham SS, et al. Systems Analysis of Immunity to Influenza Vaccination across Multiple Years and in Diverse Populations Reveals Shared Molecular Signatures. Immunity. 2015;43(6):1186-1198.

118. Khieu TQT, Pierse N, Telfar-Barnard LF, Huang QS, Baker MG. Estimating the contribution of influenza to hospitalisations in New Zealand from 1994 to 2008. Vaccine. 2015;33(33):4087-4092.

119. Brodin P, Jojic V, Gao T, et al. Variation in the human immune system is largely driven by non-heritable influences. Cell. 2015;160(1-2):37-47.

120. Carr EJ, Dooley J, Garcia-Perez JE, et al. The cellular composition of the human immune system is shaped by age and cohabitation. Nat Immunol. 2016;17(4):461-468.

121. Sasaki S, Jaimes MC, Holmes TH, et al. Comparison of the influenza 
virus-specific effector and memory B-cell responses to immunization of children and adults with live attenuated or inactivated influenza virus vaccines. J Virol. 2007;81(1):215-228.

122. Lax S, Smith DP, Hampton-Marcell J, et al. Longitudinal analysis of microbial interaction between humans and the indoor environment. Science. 2014;345(6200):1048-1052.

123. Song SJ, Lauber C, Costello EK, et al. Cohabiting family members share microbiota with one another and with their dogs. Elife. 2013;2:63786400 .

124. Mariat D, Firmesse O, Levenez F, et al. The Firmicutes/Bacteroidetes ratio of the human microbiota changes with age. BMC Microbiol. 2009;9(1):123-6.

125. Avershina E, Storrø O, Øien T, Johnsen R, Pope P, Rudi K. Major faecal microbiota shifts in composition and diversity with age in a geographically restricted cohort of mothers and their children. FEMS Microbiol Ecol. 2013;87(1):280-290.

126. Valdez Y, Brown EM, Finlay BB. Influence of the microbiota on vaccine effectiveness. Trends Immunol. 2014;35(11):526-537.

127. Harris VC, Armah G, Fuentes S, et al. Significant Correlation Between the Infant Gut Microbiome and Rotavirus Vaccine Response in Rural Ghana. J Infect Dis. 2017;215(1):34-41.

128. Human Microbiome Project Consortium. Structure, function and diversity of the healthy human microbiome. Nature. 2012;486(7402):207214.

129. Sender R, Fuchs S, Milo R. Revised Estimates for the Number of Human and Bacteria Cells in the Body. PLoS Biol. 2016;14(8):e1002533.

130. Ley RE, Bäckhed F, Turnbaugh P, Lozupone CA, Knight RD, Gordon JI. Obesity alters gut microbial ecology. Proc Natl Acad Sci USA. 2005;102(31):11070-11075.

131. Rooks MG, Garrett WS. Gut microbiota, metabolites and host immunity. Nat Rev Immunol. 2016;16(6):341-352.

132. LeBlanc JG, Milani C, de Giori GS, Sesma F, van Sinderen D, Ventura M. Bacteria as vitamin suppliers to their host: a gut microbiota perspective. Curr Opin Biotechnol. 2013;24(2):160-168. 
133. Kommineni S, Bretl DJ, Lam V, et al. Bacteriocin production augments niche competition by enterococci in the mammalian gastrointestinal tract. Nature. 2015;526(7575):719-722.

134. McKenney PT, Pamer EG. From Hype to Hope: The Gut Microbiota in Enteric Infectious Disease. Cell. 2015;163(6):1326-1332.

135. Johansson MEV, Jakobsson HE, Holmén-Larsson J, et al. Normalization of Host Intestinal Mucus Layers Requires Long-Term Microbial Colonization. Cell Host Microbe. 2015;18(5):582-592.

136. Kamada N, Seo S-U, Chen GY, Núñez G. Role of the gut microbiota in immunity and inflammatory disease. Nat Rev Immunol. 2013;13(5):321335.

137. Qin J, Li R, Raes J, et al. A human gut microbial gene catalogue established by metagenomic sequencing. Nature. 2010;464(7285):59-65.

138. Maynard CL, Elson CO, Hatton RD, Weaver CT. Reciprocal interactions of the intestinal microbiota and immune system. Nature. 2012;489(7415):231-241.

139. Suau A, Bonnet R, Sutren M, et al. Direct analysis of genes encoding $16 \mathrm{~S}$ rRNA from complex communities reveals many novel molecular species within the human gut. Appl Environ Microbiol. 1999;65(11):4799_ 4807.

140. Olsen GJ, Lane DJ, Giovannoni SJ, Pace NR, Stahl DA. Microbial ecology and evolution: a ribosomal RNA approach. Annu Rev Microbiol. 1986;40(1):337-365.

141. Jansson JK, Baker ES. A multi-omic future for microbiome studies. Nat Microbiol. 2016;1(5):16049.

142. Mowat AM, Agace WW. Regional specialization within the intestinal immune system. Nat Rev Immunol. 2014;14(10):667-685.

143. Deplancke B, Gaskins HR. Microbial modulation of innate defense: goblet cells and the intestinal mucus layer. Am J Clin Nutr. 2001;73(6):1131S-1141S.

144. Macpherson AJ, Uhr T. Compartmentalization of the mucosal immune responses to commensal intestinal bacteria. Ann $N$ Y Acad Sci. 2004;1029:36-43.

145. Atarashi K, Tanoue T, Oshima K, et al. Treg induction by a rationally 
selected mixture of Clostridia strains from the human microbiota.

Nature. 2013;500(7461):232-236.

146. Bisgaard H, Li N, Bonnelykke $\mathrm{K}$, et al. Reduced diversity of the intestinal microbiota during infancy is associated with increased risk of allergic disease at school age. J Allergy Clin Immunol. 2011;128(3):646652.

147. De Goffau MC, Luopajärvi K, Knip M, et al. Fecal microbiota composition differs between children with $\beta$-cell autoimmunity and those without. Diabetes. 2013;62(4):1238-1244.

148. Vaahtovuo J, Munukka E, Korkeamäki M, Luukkainen R, Toivanen P. Fecal microbiota in early rheumatoid arthritis. J Rheumatol. 2008;35(8):1500-1505.

149. Brenchley JM, Douek DC. Microbial translocation across the GI tract. Annu Rev Immunol. 2012;30:149-173.

150. Strober W, Fuss I, Mannon P. The fundamental basis of inflammatory bowel disease. J Clin Invest. 2007;117(3):514-521.

151. Aron-Wisnewsky J, Gaborit B, Dutour A, Clement K. Gut microbiota and non-alcoholic fatty liver disease: new insights. Clin Microbiol Infect. 2013;19(4):338-348.

152. Macfie J, O'Boyle C, Mitchell CJ, Buckley PM, Johnstone D, Sudworth P. Gut origin of sepsis: A prospective study investigating associations between bacterial translocation, gastric microflora, and septic morbidity. Gut. 1999;45(2):223-228.

153. Haas A, Zimmermann K, Graw F, et al. Systemic antibody responses to gut commensal bacteria during chronic HIV-1 infection. Gut. 2011;60(11):1506-1519.

154. Zeng MY, Cisalpino D, Varadarajan S, et al. Gut Microbiota-Induced Immunoglobulin G Controls Systemic Infection by Symbiotic Bacteria and Pathogens. Immunity. 2016;44(3):647-658.

155. Clarke TB, Davis KM, Lysenko ES, Zhou AY, Yu Y, Weiser JN. Recognition of peptidoglycan from the microbiota by Nod1 enhances systemic innate immunity. Nat Med. 2010;16(2):228-231.

156. Fagundes CT, Amaral FA, Vieira AT, et al. Transient TLR activation restores inflammatory response and ability to control pulmonary 
bacterial infection in germfree mice. J Immunol. 2012;188(3):1411-1420.

157. Ichinohe T, Pang IK, Kumamoto $Y$, et al. Microbiota regulates immune defense against respiratory tract influenza A virus infection. Proc Natl Acad Sci USA. 2011;108(13):5354-5359.

158. Oh JZ, Ravindran R, Chassaing B, et al. TLR5-Mediated Sensing of Gut Microbiota Is Necessary for Antibody Responses to Seasonal Influenza Vaccination. Immunity. 2014;41(3):478-492.

159. Balmer ML, Schürch CM, Saito $Y$, et al. Microbiota-derived compounds drive steady-state granulopoiesis via MyD88/TICAM signaling. J Immunol. 2014;193(10):5273-5283.

160. Hooper LV, Midtvedt T, Gordon JI. How host-microbial interactions shape the nutrient environment of the mammalian intestine. Annu Rev Nutr. 2002;22:283-307.

161. Tan J, McKenzie C, Potamitis M, Thorburn AN, Mackay CR, Macia L. The Role of Short-Chain Fatty Acids in Health and Disease. In: Advances in Immunology. Vol 121. Advances in Immunology. Elsevier; 2014:91119.

162. Høverstad T, Midtvedt T. Short-chain fatty acids in germfree mice and rats. J Nutr. 1986;116(9):1772-1776.

163. Trompette A, Gollwitzer ES, Yadava K, et al. Gut microbiota metabolism of dietary fiber influences allergic airway disease and hematopoiesis. Nat Med. 2014;20(2):159-166.

164. Lu Y, Fan C, Li P, Lu Y, Chang X, Qi K. Short Chain Fatty Acids Prevent High-fat-diet-induced Obesity in Mice by Regulating G Proteincoupled Receptors and Gut Microbiota. Sci Rep. November 2016:1-13.

165. Louis P, Hold GL, Flint HJ. The gut microbiota, bacterial metabolites and colorectal cancer. Nat Rev Immunol. September 2014:1-12.

166. Nakaji S, Sugawara K, Saito D, et al. Trends in dietary fiber intake in Japan over the last century. Eur J Nutr. 2002;41(5):222-227.

167. Cummings JH, Pomare EW, Branch WJ, Naylor CP, MacFarlane GT. Short chain fatty acids in human large intestine, portal, hepatic and venous blood. Gut. 1987;28(10):1221-1227.

168. McNeil NI, Cummings JH, James WPT. Short chain fatty acid absorption by the human large intestine. Gut. 1978;19(9):819-822. 
169. Le Poul E, Loison C, Struyf S, et al. Functional Characterization of Human Receptors for Short Chain Fatty Acids and Their Role in Polymorphonuclear Cell Activation. J Biol Chem. 2003;278(28):2548125489.

170. Thangaraju M, Cresci GA, Liu K, et al. GPR109A Is a G-protein-coupled receptor for the bacterial fermentation product butyrate and functions as a tumor suppressor in colon. Cancer Res. 2009;69(7):2826-2832.

171. Maciejewski-Lenoir D, Richman JG, Hakak Y, Gaidarov I, Behan DP, Connolly DT. Langerhans Cells Release Prostaglandin D2 in Response to Nicotinic Acid. J Investig Dermatol. 2006;126(12):2637-2646.

172. Maslowski KM, Vieira AT, Ng A, et al. Regulation of inflammatory responses by gut microbiota and chemoattractant receptor GPR43. Nature. 2009;461(7268):1282-1286.

173. Roediger WEW. Role of anaerobic bacteria in the metabolic welfare of the colonic mucosa in man. Gut. 1980;21(9):793-798.

174. Roediger WEW. Utilization of Nutrients by Isolated Epithelial Cells of the Rat Colon. Gastroenterology. 1982;83(2):424-429.

175. Kim M, Qie Y, Park J, Kim CH. Gut Microbial Metabolites Fuel Host Antibody Responses. Cell Host Microbe. 2016;20(2):202-214.

176. Balmer ML, Ma EH, Bantug GR, et al. Memory CD8(+) T Cells Require Increased Concentrations of Acetate Induced by Stress for Optimal Function. Immunity. 2016;44(6):1312-1324.

177. Aoyama M, Kotani J, Usami M. Butyrate and propionate induced activated or non-activated neutrophil apoptosis via HDAC inhibitor activity but without activating GPR-41/GPR-43 pathways. Nutrition. 2010;26(6):653-661.

178. Jansen MS, Nagel SC, Miranda PJ, Lobenhofer EK, Afshari CA, McDonnell DP. Short-chain fatty acids enhance nuclear receptor activity through mitogen-activated protein kinase activation and histone deacetylase inhibition. Proc Natl Acad Sci USA. 2004;101(18):7199-7204.

179. MacDonald VE, Howe LJ. Histone acetylation: where to go and how to get there. Epigenetics. 2009;4(3):139-143.

180. Park J, Kim M, Kang SG, et al. Short-chain fatty acids induce both effector and regulatory $\mathrm{T}$ cells by suppression of histone deacetylases 
and regulation of the mTOR-S6K pathway. Mucosal Immunol. 2015;8(1):80-93.

181. Arpaia N, Campbell C, Fan X, et al. Metabolites produced by commensal bacteria promote peripheral regulatory $\mathrm{T}$-cell generation. Nature. 2013;504(7480):451-455.

182. Pérez-Cano FJ, González-Castro A, Castellote C, Franch À, Castell M. Influence of breast milk polyamines on suckling rat immune system maturation. Dev Comp Immunol. 2010;34(2):210-218.

183. Sen GC. Viruses and Interferons. Vol 55. 2001:255-281.

184. Steed AL, Christophi GP, Kaiko GE, et al. The microbial metabolite desaminotyrosine protects from influenza through type I interferon. Science. 2017;357(6350):498-502.

185. Levy M, Kolodziejczyk AA, Thaiss CA, Elinav E. Dysbiosis and the immune system. Nat Rev Immunol. 2017;17(4):219-232.

186. Hicks LA, Bartoces MG, Roberts RM, et al. US outpatient antibiotic prescribing variation according to geography, patient population, and provider specialty in 2011. Clin Infect Dis. 2015;60(9):1308-1316.

187. Korpela K, Salonen A, Virta LJ, et al. Intestinal microbiome is related to lifetime antibiotic use in Finnish pre-school children. Nat Commun. 2016;7:10410.

188. Jernberg C, Löfmark S, Edlund C, Jansson JK. Long-term ecological impacts of antibiotic administration on the human intestinal microbiota. ISME J. 2007;1(1):56-66.

189. Walker AW, Ince J, Duncan SH, et al. Dominant and diet-responsive groups of bacteria within the human colonic microbiota. ISME J. 2010;5(2):220-230.

190. David LA, Maurice CF, Carmody RN, et al. Diet rapidly and reproducibly alters the human gut microbiome. Nature. 2014;505(7484):559-563.

191. Cordain L, Eaton SB, Sebastian A, et al. Origins and evolution of the Western diet: health implications for the 21st century. Am J Clin Nutr. 2005;81(2):341-354.

192. De Filippo C, Cavalieri D, Di Paola M, et al. Impact of diet in shaping gut microbiota revealed by a comparative study in children from 
Europe and rural Africa. Proc Natl Acad Sci USA. 2010;107(33):1469114696.

193. Sonnenburg ED, Smits SA, Tikhonov M, Higginbottom SK, Wingreen NS, Sonnenburg JL. Diet-induced extinctions in the gut microbiota compound over generations. Nature. 2016;529(7585):212-215.

194. Chassaing B, Koren O, Goodrich JK, et al. Dietary emulsifiers impact the mouse gut microbiota promoting colitis and metabolic syndrome. Nature. 2015;519(7541):92-96.

195. Suez J, Korem T, Zeevi D, et al. Artificial sweeteners induce glucose intolerance by altering the gut microbiota. Nature. 2014;514(7521):181186.

196. Manichanh C, Rigottier-Gois L, Bonnaud E, et al. Reduced diversity of faecal microbiota in Crohn's disease revealed by a metagenomic approach. Gut. 2006;55(2):205-211.

197. Kostic AD, Gevers D, Siljander H, et al. The dynamics of the human infant gut microbiome in development and in progression toward type 1 diabetes. Cell Host Microbe. 2015;17(2):260-273.

198. Risnes KR, Belanger K, Murk W, Bracken MB. Antibiotic exposure by 6 months and asthma and allergy at 6 years: findings in a cohort of 1,401 US children. Am J Epidemiol. 2011;173(3):310-318.

199. Carmody RN, Gerber GK, Luevano JM, et al. Diet dominates host genotype in shaping the murine gut microbiota. Cell Host Microbe. 2015;17(1):72-84.

200. Kristensen NB, Bryrup T, Allin KH, Nielsen T, Hansen TH, Pedersen O. Alterations in fecal microbiota composition by probiotic supplementation in healthy adults: a systematic review of randomized controlled trials. Genom Med. 2016;8(1):52.

201. Gibson GR, Probert HM, Loo JV, Rastall RA, Roberfroid MB. Dietary modulation of the human colonic microbiota: updating the concept of prebiotics. Nutr Res Rev. 2004;17(2):259-275.

202. Reid G, Younes JA, Van der Mei HC, Gloor GB, Knight R, Busscher HJ. Microbiota restoration: natural and supplemented recovery of human microbial communities. Nat Rev Micro. 2010;9(1):27-38.

203. Nakayama Y, Moriya T, Sakai F, et al. Oral administration of 
Lactobacillus gasseri SBT2055 is effective for preventing influenza in mice. Sci Rep. 2014;4.

204. Hori T, Kiyoshima J, Shida K, Yasui H. Augmentation of cellular immunity and reduction of influenza virus titer in aged mice fed Lactobacillus casei strain Shirota. Clin Diagn Lab Immunol. 2002;9(1):105108.

205. Rizzardini G, Eskesen D, Calder PC, Capetti A, Jespersen L, Clerici M. Evaluation of the immune benefits of two probiotic strains Bifidobacterium animalis ssp. lactis, BB-12® and Lactobacillus paracasei ssp. paracasei, L. casei $431 \circledR$ in an influenza vaccination model: a randomised, double-blind, placebo-controlled study. Br J Nutr. 2011;107(06):876-884.

206. Davidson LE, Fiorino A-M, Snydman DR, Hibberd PL. Lactobacillus GG as an immune adjuvant for live-attenuated influenza vaccine in healthy adults: a randomized double-blind placebo-controlled trial. Eur J Clin Nutr. 2011;65(4):501-507.

207. Olivares M, Díaz-Ropero MP, Sierra S, et al. Oral intake of Lactobacillus fermentum CECT5716 enhances the effects of influenza vaccination. Nutrition. 2007;23(3):254-260.

208. Boge T, Rémigy M, Vaudaine S, Tanguy J, Bourdet-Sicard R, van der Werf $S$. A probiotic fermented dairy drink improves antibody response to influenza vaccination in the elderly in two randomised controlled trials. Vaccine. 2009;27(41):5677-5684.

209. Bunout D, Barrera G, Hirsch S, et al. Effects of a nutritional supplement on the immune response and cytokine production in free-living Chilean elderly. J Parenter Enteral Nutr. 2004;28(5):348-354.

210. Lomax AR, Cheung LVY, Noakes PS, Miles EA, Calder PC. Inulin-type $\beta 2-1$ fructans have some effect on the antibody response to seasonal influenza vaccination in healthy middle-aged humans. Front Immunol. 2015;6:490.

211. Bunout D, Hirsch S, Pía de la Maza M, et al. Effects of prebiotics on the immune response to vaccination in the elderly.J Parenter Enteral Nutr. 2002;26(6):372-376.

212. Abt MC, Osborne LC, Monticelli LA, et al. Commensal bacteria calibrate the activation threshold of innate antiviral immunity. Immunity. 2012;37(1):158-170. 
213. Jonges M, Liu WM, van der Vries E, et al. Influenza virus inactivation for studies of antigenicity and phenotypic neuraminidase inhibitor resistance profiling. J Clin Microbiol. 2010;48(3):928-940.

214. Zou S, Guo J, Gao R, et al. Inactivation of the novel avian influenza A (H7N9) virus under physical conditions or chemical agents treatment. Virol J. 2013;10(1):289.

215. Yang Y-W, Chen M-K, Yang B-Y, et al. Use of 16S rRNA gene-targeted group-specific primers for real-time PCR analysis of predominant bacteria in mouse feces. Griffiths MW, ed. Appl Environ Microbiol. 2015;81(19):6749-6756.

216. Connor LM, Tang S-C, Cognard E, et al. Th2 responses are primed by skin dendritic cells with distinct transcriptional profiles. J Exp Med. 2017;214(1):125-142.

217. Nikolic T, Dingjan GM, Leenen PJM, Hendriks RW. A subfraction of $\mathrm{B} 220(+)$ cells in murine bone marrow and spleen does not belong to the $\mathrm{B}$ cell lineage but has dendritic cell characteristics. Eur J Immunol. 2002;32(3):686-692.

218. Bi S, Baum LG. Sialic acids in T cell development and function. Biochim Biophys Acta. 2009;1790(12):1599-1610.

219. Frank GM, Angeletti D, Ince WL, et al. A simple flow-cytometric method measuring $B$ cell surface immunoglobulin avidity enables characterization of affinity maturation to influenza A virus. MBio. 2015;6(4):e01156.

220. Mosiman VL, Patterson BK, Canterero L, Goolsby CL. Reducing cellular autofluorescence in flow cytometry: an in situ method. Cytometry. 1997;30(3):151-156.

221. Naik SH, Sathe P, Park H-Y, et al. Development of plasmacytoid and conventional dendritic cell subtypes from single precursor cells derived in vitro and in vivo. Nat Immunol. 2007;8(11):1217-1226.

222. Parker SCJ, Hansen L, Abaan HO, Tullius TD, Margulies EH. Local DNA topography correlates with functional noncoding regions of the human genome. Science. 2009;324(5925):389-392.

223. Cheong C, Matos I, Choi J-H, et al. Microbial stimulation fully differentiates monocytes to DC-SIGN/CD209(+) dendritic cells for immune T cell areas. Cell. 2010;143(3):416-429. 
224. Epelman S, Lavine KJ, Randolph GJ. Origin and functions of tissue macrophages. Immunity. 2014;41(1):21-35.

225. Schulz C, Gomez Perdiguero E, Chorro L, et al. A lineage of myeloid cells independent of Myb and hematopoietic stem cells. Science. 2012;336(6077):86-90.

226. Yona S, Kim K-W, Wolf $\mathrm{Y}$, et al. Fate mapping reveals origins and dynamics of monocytes and tissue macrophages under homeostasis. Immunity. 2013;38(1):79-91.

227. Iwasaki A, Medzhitov R. Control of adaptive immunity by the innate immune system. 2015;16(4):343-353.

228. Swiecki M, Miller HL, Sesti-Costa R, Cella M, Gilfillan S, Colonna M. Microbiota induces tonic CCL2 systemic levels that control pDC trafficking in steady state. Mucosal Immunol. 2017;10(4):936-945.

229. Khosravi A, Yáñez A, Price JG, et al. Gut microbiota promote hematopoiesis to control bacterial infection. Cell Host Microbe. 2014;15(3):374-381.

230. Liu K, Waskow C, Liu X, Yao K, Hoh J, Nussenzweig M. Origin of dendritic cells in peripheral lymphoid organs of mice. Nat Immunol. 2007;8(6):578-583.

231. Young MH. T cell receptor signaling and Toll-like receptor signaling converge to amplify T cell responses. J Immunol. 196(1 Supplement):128.5.

232. Round JL, Lee SM, Li J, et al. The Toll-Like Receptor 2 Pathway Establishes Colonization by a Commensal of the Human Microbiota. 2011;332(6032):974-977.

233. Kubinak JL, Petersen C, Stephens WZ, et al. MyD88 signaling in T cells directs IgA-mediated control of the microbiota to promote health. Cell Host Microbe. 2015;17(2):153-163.

234. Pasare C, Medzhitov R. Control of B-cell responses by Toll-like receptors. Nature. 2005;438(7066):364-368.

235. Blaser MJ. Who are we? Indigenous microbes and the ecology of human diseases. EMBO Rep. 2006;7(10):956-960.

236. Ma A, Han X, Zhang Y, Gao Y-H, Lan J. Effect of kiwifruit extract supplementation on levels of serum immunoglobulins and 
phagocytosis activity in mice. FASEB J. 2006;20(5):A1057-A1057.

237. Hunter DC, Denis M, Parlane NA, Buddle BM, Stevenson LM, Skinner MA. Feeding ZESPRI GOLD Kiwifruit puree to mice enhances serum immunoglobulins specific for ovalbumin and stimulates ovalbuminspecific mesenteric lymph node cell proliferation in response to orally administered ovalbumin. Nutr Res. 2008;28(4):251-257.

238. Shu Q, Mendis De Silva U, Chen S, et al. Kiwifruit extract enhances markers of innate and acquired immunity in a murine model. Food Agric Immunol. 2008;19(2):149-161.

239. Hunter DC, Skinner MA, Wolber FM, et al. Consumption of gold kiwifruit reduces severity and duration of selected upper respiratory tract infection symptoms and increases plasma vitamin $\mathrm{C}$ concentration in healthy older adults. Br J Nutr. 2012;108(7):1235-1245.

240. Ansell J, Drummond LN. Establishing the prebiotic potential of whole kiwifruit. Agro Food Industry Hi-Tech. 2011;22(6):14-16.

241. Veiga P, Pons N, Agrawal A, et al. Changes of the human gut microbiome induced by a fermented milk product. Sci Rep. 2014;4(1):6328-209.

242. Park YK, Alencar SM, Aguiar CL. Botanical origin and chemical composition of Brazilian propolis. J Agric Food Chem. 2002;50(9):25022506.

243. Cardona F, Andrés-Lacueva C, Tulipani S, Tinahones FJ, QueipoOrtuño MI. Benefits of polyphenols on gut microbiota and implications in human health. J Nutr Biochem. 2013;24(8):1415-1422.

244. Wang K, Jin X, You M, et al. Dietary Propolis Ameliorates Dextran Sulfate Sodium-Induced Colitis and Modulates the Gut Microbiota in Rats Fed a Western Diet. 2017;9(8):875-813.

245. Tumpey TM, Basler CF, Aguilar PV, et al. Characterization of the reconstructed 1918 Spanish influenza pandemic virus. Science. 2005;310(5745):77-80.

246. Tate MD, Ioannidis LJ, Croker B, Brown LE, Brooks AG, Reading PC. The Role of Neutrophils during Mild and Severe Influenza Virus Infections of Mice. Fouchier R, ed. PLoS ONE. 2011;6(3):e17618-10.

247. Minne A, Louahed J, Mehauden S, Baras B, Renauld J-C, Vanbever R. 
The delivery site of a monovalent influenza vaccine within the respiratory tract impacts on the immune response. Immunology. 2007;122(3):316-325.

248. Tumpey TM, Renshaw M, Clements JD, Katz JM. Mucosal delivery of inactivated influenza vaccine induces B-cell-dependent heterosubtypic cross-protection against lethal influenza A H5N1 virus infection. J Virol. 2001;75(11):5141-5150.

249. Reber AJ, Kim JH, Biber R, et al. Preexisting Immunity, More Than Aging, Influences Influenza Vaccine Responses. Open Forum Infect Dis. 2015;2(2):1-12.

250. Fan Y-C, Chiu H-C, Chen L-K, Chang G-JJ, Chiou S-S. Formalin Inactivation of Japanese Encephalitis Virus Vaccine Alters the Antigenicity and Immunogenicity of a Neutralization Epitope in Envelope Protein Domain III. PLoS Negl Trop Dis. 2015;9(10):e0004167.

251. Maldonado-Gómez MX, Martínez I, Bottacini F, et al. Stable Engraftment of Bifidobacterium longum AH1206 in the Human Gut Depends on Individualized Features of the Resident Microbiome. Cell Host Microbe. 2016;20(4):515-526.

252. Nguyen TLA, Vieira-Silva S, Liston A, Raes J. How informative is the mouse for human gut microbiota research? Dis Model Mech. 2015;8(1):116.

253. Sadler R, Singh V, Benakis C, et al. Microbiota differences between commercial breeders impacts the post-stroke immune response. Brain, Behavior, and Immunity. 2017;66:23-30.

254. Sanchez HN. Short-chain fatty acid (SCFA) histone deacetylase inhibitors produced by gut microbiota regulate selected microRNAs to modulate local and systemic antibody responses. J Immunol. 196(1 Supplement):127.6.

255. Wollenberg I, Agua-Doce A, Hernandez A, et al. Regulation of the germinal center reaction by Foxp3+ follicular regulatory $\mathrm{T}$ cells. $J$ Immunol. 2011;187(9):4553-4560.

256. Aloulou M, Carr EJ, Gador M, et al. Follicular regulatory T cells can be specific for the immunizing antigen and derive from naive T cells. Nat Commun. 2016;7:10579.

257. Spears JK, Karr-Lilienthal LK, Fahey GC Jr. Influence of supplemental 
high molecular weight pullulan or $\gamma$-cyclodextrin on ileal and total tract nutrient digestibility, fecal characteristics, and microbial populations in the dog. Arch Anim Nutr. 2005;59(4):257-270.

258. Webster RG, Askonas BA. Cross-protection and cross-reactive cytotoxic T cells induced by influenza virus vaccines in mice. Eur J Immunol. 1980;10(5):396-401.

259. Ennis FA, Martin WJ, Verbonitz MW. Cytotoxic T lymphocytes induced in mice by inactivated influenza virus vaccine. Nature. 1977;269(5627):418-419.

260. Clarke TB. Early innate immunity to bacterial infection in the lung is regulated systemically by the commensal microbiota via nod-like receptor ligands. Bäumler AJ, ed. Infect Immun. 2014;82(11):4596-4606.

261. Ganal SC, Sanos SL, Kallfass C, et al. Priming of natural killer cells by nonmucosal mononuclear phagocytes requires instructive signals from commensal microbiota. Immunity. 2012;37(1):171-186.

262. Macpherson AJ, Uhr T. Induction of protective IgA by intestinal dendritic cells carrying commensal bacteria. Science. 2004;303(5664):1662-1665.

263. Thorburn AN, McKenzie CI, Shen S, et al. Evidence that asthma is a developmental origin disease influenced by maternal diet and bacterial metabolites. Nat Commun. 2015;6:1-13.

264. Teng F, Klinger CN, Felix KM, et al. Gut microbiota drive autoimmune arthritis by promoting differentiation and migration of Peyer's patch $\mathrm{T}$ follicular helper cells. Immunity. 2016;44(4):875-888.

265. Jaakkola I, Jalkanen S, Hänninen A. Diabetogenic T cells are primed both in pancreatic and gut-associated lymph nodes in NOD mice. Eur J Immunol. 2003;33(12):3255-3264.

266. Hänninen A, Salmi M, Simell O, Jalkanen S. Mucosa-associated ( $\beta 7$ integrin(high)) lymphocytes accumulate early in the pancreas of NOD mice and show aberrant recirculation behavior. Diabetes. 1996;45(9):1173-1180.

267. Pabst O, Hornef M. Gut microbiota: a natural adjuvant for vaccination. Immunity. 2014;41(3):349-351.

268. Naik S, Bouladoux N, Wilhelm C, et al. Compartmentalized control of 
skin immunity by resident commensals. Science. 2012;337(6098):11151119.

269. Oudemans-van Straaten HM, van Saene HKF, Zandstra DF. Selective decontamination of the digestive tract: use of the correct antibiotics is crucial. Crit Care Med. 2003;31(1):334-335.

270. Buchta CM, Bishop GA. Toll-like receptors and B cells: functions and mechanisms. Immunol Res. 2014;59(1-3):12-22.

271. Coffman RL, Lebman DA, Shrader B. Transforming growth factor beta specifically enhances IgA production by lipopolysaccharide-stimulated murine B lymphocytes. J Exp Med. 1989;170(3):1039-1044.

272. Griffin NW, Ahern PP, Cheng J, et al. Prior Dietary Practices and Connections to a Human Gut Microbial Metacommunity Alter Responses to Diet Interventions. Cell Host Microbe. 2017;21(1):84-96.

273. Toyoda A, Shimonishi H, Sato M, Usuda K, Ohsawa N, Nagaoka K. Effects of non-purified and semi-purified commercial diets on behaviors, plasma corticosterone levels, and cecum microbiome in C57BL/6J mice. Neurosci Lett. 2018;670:36-40.

274. Tap J, Furet J-P, Bensaada M, et al. Gut microbiota richness promotes its stability upon increased dietary fibre intake in healthy adults. Environ Microbiol. 2015;17(12):4954-4964.

275. Zeevi D, Korem T, Miyakawa M, et al. Personalized Nutrition by Prediction of Glycemic Responses. Cell. 2015;163(5):1079-1095.

276. Geva-Zatorsky N, Sefik E, Kua L, et al. Mining the human gut microbiota for immunomodulatory organisms. Cell. 2017;168(5):928943.e11.

277. Elinav E, Strowig T, Kau AL, et al. NLRP6 inflammasome regulates colonic microbial ecology and risk for colitis. Cell. 2011;145(5):745-757. 